\title{
Pressure of a weakly magnetized hot and dense deconfined QCD matter in one-loop hard-thermal-loop perturbation theory
}

\author{
Aritra Bandyopadhyay, ${ }^{1,2, *}$ Bithika Karmakar, ${ }^{1, \dagger}$ Najmul Haque, ${ }^{3,4, \$}$ and Munshi G. Mustafa ${ }^{1, \S}$ \\ ${ }^{1}$ Theory Division, Saha Institute of Nuclear Physics, HBNI, 1/AF, Bidhannagar, Kolkata 700064, India \\ ${ }^{2}$ Departamento de Física, Universidade Federal de Santa Maria, Santa Maria, RS 97105-900, Brazil \\ ${ }^{3}$ School of Physical Sciences, National Institute of Science Education and Research, \\ HBNI, Jatni, Khurda 752050, India \\ ${ }^{4}$ Institut für Theoretische Physik, Justus-Liebig-Universität Giessen, 35392 Giessen, Germany
}

(Received 20 November 2018; published 30 August 2019)

\begin{abstract}
We consider our recently obtained general structure of two point (self-energy and propagator) functions of quarks and gluons in a nontrivial background like a heat bath and an external magnetic field. Based on this, here we have computed the free energy and pressure of quarks and gluons for a magnetized hot and dense deconfined QCD matter in weak field approximation. For a heat bath we have used hard-thermal-loop perturbation theory (HTLpt) in the presence of a finite chemical potential. For weak field approximations we have obtained the pressure of QCD matter, both with and without the high temperature expansion. The results with high $T$ expansions are completely analytic and gauge independent but depend on the renormalization scale in addition to the temperature, chemical potential, and the external magnetic field. We also discuss the modification of QCD Debye mass of such matter for an arbitrary magnetic field. Analytic expressions for Debye mass are also obtained for both strong and weak field approximations. It is found to exhibit some interesting features depending upon the three different scales, i.e., the quark mass, temperature, and the strength of the magnetic field. The various divergences appearing in the quark and gluon free energies are regulated through appropriate counterterms. In weak field approximation, the low temperature behavior of the pressure is found to strongly depend on the magnetic field more so than that at high temperature. We also discuss the specific problem with one-loop HTLpt associated with the over-counting of certain orders in coupling.
\end{abstract}

DOI: 10.1103/PhysRevD.100.034031

\section{INTRODUCTION}

Quark gluon plasma (QGP) is a thermalized color deconfined state of nuclear matter in the regime of quantum chromodynamics (QCD) under extreme conditions such as very high temperature and/or density. For the past couple of decades, different high energy heavy-ion-collisions (HIC) experiments are under way, e.g., RHIC at BNL, LHC at CERN, and the upcoming FAIR at GSI, to study this novel state of QCD matter within the largely unknown QCD phase diagram. In recent years the focus has also shifted toward the noncentral HIC, where a very strong magnetic field is created in the direction perpendicular to the reaction plane due to the spectator particles that are not participating in the

\footnotetext{
*aritrabanerjee.444@gmail.com

bithika.karmakar@saha.ac.in

*nhaque@niser.ac.in

${ }^{\S}$ munshigolam.mustafa@saha.ac.in
}

Published by the American Physical Society under the terms of the Creative Commons Attribution 4.0 International license. Further distribution of this work must maintain attribution to the author(s) and the published article's title, journal citation, and DOI. Funded by SCOAP ${ }^{3}$. collisions [1-5]. Recent experimental evidences of photon anisotropy provided by the PHENIX Collaboration [6] has also challenged the present theoretical tools. By assuming a presence of large anisotropic magnetic field generated in HIC, eventually some explanations were made [7] in support of those experimental findings. In fact this has prompted that a theoretical study is much needed by considering the effects of an intense background magnetic field on various aspects and observables of noncentral HIC. Also some of these studies have subsequently revealed that the strong magnetic field generated during the noncentral $\mathrm{HIC}$ is also time dependent. More specifically, it rapidly decreases with time $[8,9]$. Nevertheless, the inclusion of an external magnetic field in QGP introduces also an extra energy scale in the system. At the time of the noncentral HIC, the value of the created magnetic field $B$ is very high compared to the temperature $T\left(T^{2}<q_{f} B\right.$ where $q_{f}$ is the absolute charge of the quark with flavor $f$ ) associated with the system. It is estimated up to the order of $q_{f} B \sim 15 m_{\pi}^{2}$ in the LHC at CERN [10]. On the other hand, neutron stars (NS), or more specifically magnetars, are also known to possess a strong enough magnetic field [11-13]. In this regime of study, one usually works in the strong magnetic field approximation. 
The presence of an external anisotropic field in the medium calls for the appropriate modification of the present theoretical tools to investigate various properties of QGP, and a lot of activities are in progress. Over the last few years, several novel phenomena came into light, e.g., chiral magnetic effect [14-16], magnetic catalysis [17-19], and inverse magnetic catalysis [20-27] at finite temperature; chiral- and color-symmetry broken/restoration phase [28-32], thermodynamic properties [31-35], refractive indices and decay constant of hadrons [36-40] and the equation of state (EOS) in holographic models [41,42] in a hot magnetized medium; soft photon production from conformal anomaly [7,43] in HIC; modification of dispersion properties in a magnetized hot QED [44] and QCD [45-48] medium; and various transport coefficients [49-51], properties of quarkonia [52,53], synchroton radiation [54], and dilepton production from a hot magnetized QCD plasma [54-59] and in strongly coupled plasma in a strong magnetic field [60].

Thermodynamic properties of low lying hadrons in the presence of a magnetic field have been studied in recent years within the various hadronic models [31-34]. Nevertheless, the EOS is a generic quantity and of phenomenological importance for studying the hot and dense QCD matter, QGP, created in HIC. At zero chemical potential and finite temperature, lattice QCD (LQCD) established itself as the most reliable method to calculate thermodynamic functions. Unfortunately at finite chemical potential LQCD faces the infamous sign problem. Information about the thermodynamic functions in LQCD can still be extracted by making a Taylor expansion of the partition function around zero baryonic chemical potential and extrapolating the result [61]. But due to the finite number of Taylor coefficients, such extrapolation has its own limitations. On the other hand, naively, the asymptotic freedom of QCD leads us to expect that bare perturbation theory should be a reliable guide to calculate these properties of matter at high temperature and/or high density [62-68]. Although, it has been recognized early on that this is not so. Technically, infrared divergences plague the calculation of observables at finite temperature, preventing the determination of high order corrections. In order to cope with this difficulty, whose origin is the presence of massless particles, it has been suggested to reorganize perturbation theory, by performing the expansion around of a system of massive quasiparticles. The motivation for doing so is that thermal fluctuations can generate a mass. It amounts to a resummation of a class of loop diagrams, where the loop momenta are of the order of the temperature. Such diagrams are those which contribute to give the excitations a thermal mass. The hard-thermalloop perturbation theory (HTLpt) is one such state-of-theart resummed perturbation theory [69]. In HTLpt the EOS of QCD in absence of a magnetic field has systematically been computed within one-loop [leading order (LO)]
[69-77], two loop [next-to-leading order (NLO)] [78-81] and three loop [next-to-next-to-leading order (NNLO)] [82-88] at finite temperature and chemical potential. Although the all-loop order calculations are gauge invariant, the three-loop results are complete in $g^{5}$ and fully analytic which do not require any free fit parameters besides a renormalization scale. The thermomagnetic correction to the quark-gluon vertex in the presence of a weak magnetic field within the HTL approximation has recently been computed $[89,90]$. Also recently, the general structure of gluon $[45,46,48]$ and quark [47] self-energy and propagator and their spectra have been obtained in a thermomagnetic medium within HTL approximation. Further, the thermodynamic quantities in lowest Landau level (LLL) within the strong field approximation has been calculated in Ref. [35] using HTL approximation. But in this calculation the change in the general structure of two point functions of a gluon has not been considered. It assumes for the gluonic case without any justification that the shift in the Debye mass is the only effect of the magnetic field. However, this is not the case as it has explicitly been shown in paper-I [45] that the presence of an external magnetic field breaks the rotational symmetry.

In view of this, presently, a systematic determination of EOS for magnetized hot QCD medium is of great importance. In this article (say paper-II), ${ }^{1}$ we make an effort to derive the pressure of a magnetized hot and dense deconfined QCD medium created in high energy HIC. Usually two kinds of approach were taken in all of the previous studies of EOS in the presence of a magnetic field. In the first kind, the pressure remains isotropic and the system can be easily described in terms of standard thermodynamic relations $[12,13]$. In the second one, the breaking of the spherical symmetry due to the anisotropic background magnetic field in a preferred direction [91-95] is taken into consideration. Subsequently, this results in an anisotropic pressure arising from the difference between pressure components that are transverse and longitudinal to the background magnetic field direction. Eventually, the difference in stress causes the deformation of the fireball produced in heavy ion collisions or the NS. There are also some recent LQCD calculations, which incorporate both of these schemes [96]. However, it is also shown in [33] that pressure anisotropy decreases with the increase in temperature. Moreover, the magnetic field created in noncentral HIC is a fast decreasing function of time [8,9]; it is expected that by the time the quarks and gluons thermalize in a QGP medium, the magnetic field strength becomes sufficiently weak. By virtue of which, temperature at that time becomes the largest energy scale of the system. In this regime, in principle, one can work within the weak magnetic field approximation in which the pressure can

\footnotetext{
${ }^{1}$ Paper-I [45].
} 
be considered isotropic. ${ }^{2}$ In this paper II we work on the weak $\left(q_{f} B<m_{f}^{2} \sim m_{\mathrm{th}}^{2} \sim g^{2} T^{2}<T^{2}\right)$ field limit where $m_{f}$ is the mass of fermion, $m_{\text {th }}$ is the thermal mass of a fermion, $T$ is the temperature, $g$ is the strong coupling, and $B$ is the strength of an external magnetic field. For the strong field case, the system is considered to be confined in the lowest Landau level and the transverse pressure vanishes in the LLL or in the Landau ground state due to dimensional reduction. We note that the pressure along the field direction, i.e., longitudinal pressure in a strong field approximation $\left(m_{f}^{2} \sim m_{\mathrm{th}}^{2} \sim g^{2} T^{2}<T^{2}<q_{f} B\right)$, has already been computed in Ref. [35]. This is because the average transverse momentum of fermion vanishes as the strength of $B$ increases. On the other hand, a weak magnetic field case is different than that of a strong field one, as we shall see below.

In this paper-II, for the first time, by considering the general two point function of a quark [47] and a gluon [45] in a hot but weakly magnetized deconfined QCD medium, we shall compute the pressure of quark-gluon plasma within HTL approximation. As we would see, the calculation is very involved; nevertheless the expression of free energy vis a vis pressure is completely analytic and gauge independent. We have used a strong coupling that runs through the renormalization scale and strength of the magnetic field in a weak field domain. Sensitivity of the two scales on the pressure has also been discussed in details.

This paper has been organized as follows: In Sec. II the basic computation of the paper is briefly outlined. The scale hierarchies in weak field approximation are discussed in Sec. III. In Sec. IV we discuss the one-loop quark free energy for a magnetized hot QCD medium within weak field and HTL approximations by considering the general structure of the quark propagator [47]. Section V discusses gluon free energy for a magnetized hot and dense QCD medium in terms of the general structure of two point functions of a gauge boson as obtained in paper-I [45]. In Sec. VA we discuss the Debye screening mass both in strong and weak field approximations that clearly separates the two domains. In Sec. VI we obtain the finite contribution of weak field free energy in Sec. VI A and pressure in Sec. VI B of a magnetized hot medium. The magnetic field dependent strong coupling is discussed in Sec. VII and its validity in a weak field domain is justified. In Sec. VIII we compare approximate and full results thereby justifying the use of the former. The main results with high $T$ expansion are discussed in Sec. IX and finally, we conclude in Sec. X. The detailed calculations associated with various sections are given in Appendices A, B, C, and D and various subsections therein.

\section{SETUP}

The total thermodynamic free energy up to one-loop order in HTLpt in the presence of a background magnetic field, $B$, can be written as

$$
F=F_{q}+F_{g}+F_{0}+\Delta \mathcal{E}_{0},
$$

where $F_{q}$ and $F_{g}$ are, respectively, the quark and gluon part of the free energy which will be computed in the presence of a magnetic field with HTL approximation. $F_{0}=\frac{1}{2} B^{2}$ is the tree-level contribution due to the constant magnetic field and the $\Delta \mathcal{E}_{0}$ is the HTL counterterm given [80] as

$$
\Delta \mathcal{E}_{0}=\frac{d_{A}}{128 \pi^{2} \epsilon} m_{D}^{4}
$$

with $d_{A}=N_{c}^{2}-1, N_{c}$ is the number of color in fundamental representation, and $m_{D}$ is the Debye screening mass in HTL approximation.

The pressure of a system is defined as

$$
P=-F \text {. }
$$

\section{SCALE HIERARCHIES IN WEAK FIELD APPROXIMATION}

The magnetic field generated in heavy-ion collisions decreases rapidly with time. This provides us a simplified situation where one can work in weak field approximation because temperature is the highest scale in the system. The presence of magnetic field $q_{f} B$ introduces another scale in addition to the thermal scales $g T$ and $T$. In weak field approximation one can have two hierarchies of scales:

(i) When $\sqrt{q_{f} B}$ is the smallest scale compared to temperature and quark mass, one can work with a hierarchy of scales $\sqrt{q_{f} B}<m_{f}<T$ and $\sqrt{q_{f} B}$ can be treated as perturbation. This allows one to expand, for example, the Schwinger propagator for a fermion in weak field approximation $[98,99]$ up to an $\mathcal{O}\left[\left(q_{f} B\right)^{2}\right]$ as

$$
\begin{aligned}
i S_{m}^{w}(k)= & i \frac{K+m_{f}}{K^{2}-m_{f}^{2}}\left(q_{f} B\right)^{0}+i\left(q_{f} B\right) \frac{\left(\gamma_{5}\{(K \cdot n) \not h-(K \cdot u) \not h\}+i \gamma_{1} \gamma_{2} m_{f}\right)}{\left(K^{2}-m_{f}^{2}\right)^{2}} \\
& +i 2\left(q_{f} B\right)^{2}\left[\frac{\{(K \cdot u) \not h-(K \cdot n) \not h\}-\not K}{\left(K^{2}-m_{f}^{2}\right)^{3}}-\frac{k_{\perp}^{2}\left(K+m_{f}\right)}{\left(K^{2}-m_{f}^{2}\right)^{4}}\right]+\mathcal{O}\left[\left(q_{f} B\right)^{3}\right],
\end{aligned}
$$

\footnotetext{
${ }^{2}$ However, one should consider anisotropic pressure, i.e., different pressures along the longitudinal and the transverse directions in the case of a strong magnetic field approximation [97].
} 
which is a perturbative series of $q_{f} B$. In $q_{f} B \rightarrow 0$, the thermomagnetic correction vanishes. Alternatively, the thermomagnetic effects are obtained as higher order perturbative corrections to the nonmagnetized part [i.e., HTL part as $\left.\left(q_{f} B\right)^{0}\right]$. This means that for each given order in $q_{f} B$ in a perturbative series, one can use HTL approximation within the scale hierarchy $\sqrt{q_{f} B}<g T<T$ to obtain the desired order of coupling.

(ii) When quark mass $m_{f}$ is the smallest scale compared to temperature and magnetic field, one may work with a hierarchy $m_{f}<\sqrt{q_{f} B}<T$ by considering $m_{f}$ as perturbation for a given order of $q_{f} B$. In this hierarchy $m_{f}$ in a fermion propagator is either set to be zero or expanded in $m_{f}$ for a given order of $q_{f} B$.

As discussed above, we will be working only with the hierarchy ${ }^{3} \sqrt{q_{f} B}<m_{f}<T$ in this paper.

\section{QUARK FREE ENERGY IN THE PRESENCE OF WEAK MAGNETIC FIELD}

\section{A. General structure of two-point fermionic function}

The inverse of the effective fermion propagator following the Dyson-Schwinger equation, as given in Fig. 1, can now be written as

$$
S_{\text {eff }}^{-1}(P)=\not P-\Sigma(P) .
$$

The general structure of a fermionic two-point function and its dispersion spectrum in a hot magnetized medium has recently been discussed in detail in Ref. [47]. The most general form of the fermion self-energy for $\mathrm{CPT}^{4}$ and chirally invariant theory in a hot magnetized medium becomes

$$
\Sigma(P)=-\mathcal{A} \not P-\mathcal{B} \not h-\mathcal{B}^{\prime} \gamma_{5} \not h-\mathcal{C}^{\prime} \gamma_{5} \not h,
$$

where $u_{\mu}$ is the four velocity of the heat bath and the direction of the magnetic field $[45,47] n_{\mu}$ is given as

$$
n_{\mu}=\frac{1}{2 B} \epsilon_{\mu \nu \rho \lambda} u^{\nu} F^{\rho \lambda}=\frac{1}{B} u^{\nu} \tilde{F}_{\mu \nu} .
$$

The background dual field tensor $\tilde{F}_{\mu \nu}$ can be written in terms of field tensor $F_{\mu \nu}$ as

$$
\tilde{F}_{\mu \nu}=\frac{1}{2} \epsilon_{\mu \nu \rho \lambda} F^{\rho \lambda} .
$$

Without any loss of generality we have considered the four velocity in the rest frame of the heat bath and the direction of the magnetic field $B$ along the $z$ direction. So,

\footnotetext{
${ }^{3}$ Calculation with the other hierarchy is itself an independent problem.

${ }^{4}$ Charge conjugation, parity, and time reversal.
}

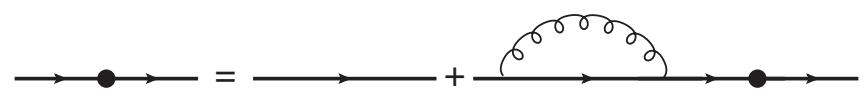

FIG. 1. Diagrammatic representation of the Dyson-Schwinger equation for one-loop effective fermion propagator.

$$
\begin{aligned}
& u^{\mu}=(1,0,0,0), \\
& n_{\mu}=(0,0,0,1) .
\end{aligned}
$$

The general form of the various structure functions can be obtained from Eq. (6) as

$$
\begin{gathered}
\mathcal{A}=\frac{1}{4} \frac{\operatorname{Tr}[\Sigma(P) \not P]-(P \cdot u) \operatorname{Tr}[\Sigma(P) \not u]}{(P \cdot u)^{2}-P^{2}}, \\
\mathcal{B}=\frac{1}{4} \frac{-(P \cdot u) \operatorname{Tr}[\Sigma(P) \not P]+P^{2} \operatorname{Tr}[\Sigma(P) \not \ell]}{(P \cdot u)^{2}-P^{2}}, \\
\mathcal{B}^{\prime}=-\frac{1}{4} \operatorname{Tr}\left[\not \iota \Sigma(P) \gamma_{5}\right], \\
\mathcal{C}^{\prime}=\frac{1}{4} \operatorname{Tr}\left[\not h \Sigma(P) \gamma_{5}\right],
\end{gathered}
$$

which are also Lorentz scalars. Beside $T, \mu$, and $B$, these structure functions would also depend on three Lorentz scalars due to the breaking of both Lorentz (boost) and rotational invariance defined by

$$
\begin{gathered}
p_{0}=\omega \equiv P^{\mu} u_{\mu}, \\
p^{3} \equiv-P^{\mu} n_{\mu}=p_{z}, \\
p_{\perp} \equiv\left[\left(P^{\mu} u_{\mu}\right)^{2}-\left(P^{\mu} n_{\mu}\right)^{2}-\left(P^{\mu} P_{\mu}\right)\right]^{1 / 2} \\
=\left[p_{0}^{2}-p_{3}^{2}-P^{2}\right]^{1 / 2}=\left[p_{1}^{2}+p_{2}^{2}\right]^{1 / 2} .
\end{gathered}
$$

All these structure functions in Eq. (10a) to (10d) in oneloop order as shown in Fig. 2 within weak field and HTL approximations have been computed ${ }^{5}$ in Ref. [47] as

$$
\begin{gathered}
\mathcal{A}\left(p_{0}, p_{\perp}, p_{3}\right)=-\frac{m_{\mathrm{th}}^{2}}{p^{2}} \int \frac{d \Omega}{4 \pi} \frac{\boldsymbol{p} \cdot \hat{\boldsymbol{k}}}{P \cdot \hat{K}}, \\
\mathcal{B}\left(p_{0}, p_{\perp}, p_{3}\right)=\frac{m_{\mathrm{th}}^{2}}{p^{2}} \int \frac{d \Omega}{4 \pi} \frac{(P \cdot u)(\boldsymbol{p} \cdot \hat{\boldsymbol{k}})-p^{2}}{P \cdot \hat{K}}, \\
\mathcal{B}^{\prime}\left(p_{0}, p_{\perp}, p_{3}\right)=-m_{\mathrm{eff}}^{2} \int \frac{d \Omega}{4 \pi} \frac{\hat{K} \cdot n}{P \cdot \hat{K}}, \\
\mathcal{C}^{\prime}\left(p_{0}, p_{\perp}, p_{3}\right)=m_{\mathrm{eff}}^{2} \int \frac{d \Omega}{4 \pi} \frac{\hat{K} \cdot u}{P \cdot \hat{K}} .
\end{gathered}
$$

\footnotetext{
${ }^{5}$ In Ref. [47] those structure functions were computed for $\mu=0$ but we have modified it for $\mu \neq 0$.
} 


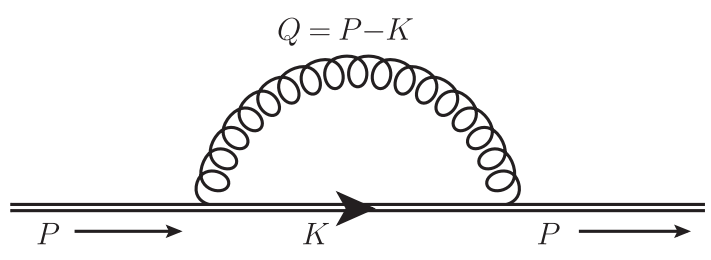

FIG. 2. Self-energy diagram for a quark in weak magnetic field approximation. The double line indicates the modified quark propagator in the presence of a magnetic field.

We emphasize that the structure functions in Eqs. (12c) and (12d) have become anisotropic in nature due to the breaking of the rotational invariance in the presence of a magnetic field in a given direction. Also note that

$$
\begin{array}{r}
m_{\mathrm{th}}^{2}=\frac{g^{2} C_{F} T^{2}}{8}\left(1+4 \hat{\mu}^{2}\right), \\
m_{\mathrm{eff}}^{2}=4 g^{2} C_{F} M_{B, f}^{2}\left(T, \mu, m_{f}, q_{f} B\right) ; \\
M_{B}^{2}=\sum_{f} M_{B, f}^{2}\left(T, \mu, m_{f}, q_{f} B\right) .
\end{array}
$$

The magnetic $\operatorname{mass}^{6}$ for a given flavor $f$ is given as

$$
M_{B, f}^{2}=\frac{q_{f} B}{16 \pi^{2}}\left[-\frac{1}{4} \aleph(z)-\frac{\pi T}{2 m_{f}}-\frac{\gamma_{E}}{2}\right],
$$

where the function $\aleph(z)$ is defined in Eq. (A14). Now we note that in the limit of a small current quark mass $\left(m_{f} \rightarrow 0\right)$, the magnetic mass in (15) diverges. It has been regulated by using the prescription given in Refs. [100,101] with a mass cutoff which is the thermal mass $m_{\text {th }}$ of the fermion.

Combining Eq. (6) with Eqs. (12a)-(12d) the total quark self-energy contribution of $\mathcal{O}\left[q_{f} B\right]$ in the presence of a weak magnetic field within HTL approximation can be written as

$$
\begin{aligned}
\Sigma(P)= & m_{\mathrm{th}}^{2} \int \frac{d \Omega}{4 \pi} \frac{\hat{K}}{P \cdot \hat{K}} \\
& +m_{\mathrm{eff}}^{2} \int \frac{d \Omega}{4 \pi} \frac{[(\hat{K} \cdot n) \not h-(\hat{K} \cdot u) \not h]}{P \cdot \hat{K}} .
\end{aligned}
$$

\footnotetext{
${ }^{6}$ In the case of a finite chemical potential the expression for $f_{1}$, the well-known fermionic function also given in Eq. (34) of Ref [90], gets modified as$$
f_{1}(y)=-\frac{1}{2} \ln \left(\frac{y}{4 \pi}\right)+\frac{1}{4} \aleph(z)+\cdots,
$$

which gets reflected in the expression of the magnetic mass in (15). For zero chemical potential the expression for magnetic mass becomes [90] $M_{B, f}^{2}=\frac{q_{f} B}{16 \pi^{2}}\left[\ln 2-\frac{\pi T}{2 m_{f}}\right]$.
}

Using the general structure of the quark self-energy and considering the external anisotropic weak magnetic field along the $z$ (or 3) direction [47] one can now write

$$
\begin{aligned}
S_{\text {eff }}^{-1}= & \not P-\Sigma(P) \\
= & {\left[\mathcal{C}\left(p_{0}, p_{\perp}, p_{3}\right) p_{0} \gamma_{0}-\mathcal{D}\left(p_{0}, p_{\perp}, p_{3}\right) p_{i} \gamma_{i}\right.} \\
& \left.+\mathcal{B}^{\prime}\left(p_{0}, p_{\perp}, p_{3}\right) \gamma_{5} \gamma_{0}+\mathcal{C}^{\prime}\left(p_{0}, p_{\perp}, p_{3}\right) \gamma_{5} \gamma_{3}\right]
\end{aligned}
$$

Here

$$
\begin{aligned}
& \mathcal{C}\left(p_{0}, p_{\perp}, p_{3}\right)=1+\mathcal{A}\left(p_{0}, p_{\perp}, p_{3}\right)+\frac{\mathcal{B}\left(p_{0}, p_{\perp}, p_{3}\right)}{p_{0}} \\
&=1-\mathcal{A}^{\prime}\left(p_{0}, p_{\perp}, p_{3}\right), \\
& \mathcal{D}\left(p_{0}, p_{\perp}, p_{3}\right)=1+\mathcal{A}\left(p_{0}, p_{\perp}, p_{3}\right),
\end{aligned}
$$

with

$\mathcal{A}\left(p_{0}, p_{\perp}, p_{3}\right)=-\frac{m_{\mathrm{th}}^{2}}{p^{2}} \int \frac{d \Omega}{4 \pi} \frac{\boldsymbol{p} \cdot \hat{\boldsymbol{k}}}{p_{0}-\boldsymbol{p} \cdot \hat{\boldsymbol{k}}}=\frac{m_{\mathrm{th}}^{2}}{p^{2}}\left[1-\mathcal{T}_{P}\right]$,

$\mathcal{A}^{\prime}\left(p_{0}, p_{\perp}, p_{3}\right)=\frac{m_{\mathrm{th}}^{2}}{p_{0}} \int \frac{d \Omega}{4 \pi} \frac{1}{p_{0}-\boldsymbol{p} \cdot \hat{\boldsymbol{k}}}=\frac{m_{\mathrm{th}}^{2}}{p_{0}^{2}} \mathcal{T}_{P}$

$\mathcal{B}^{\prime}\left(p_{0}, p_{\perp}, p_{3}\right)=-m_{\mathrm{eff}}^{2} \int \frac{d \Omega}{4 \pi} \frac{\hat{k_{3}}}{p_{0}-\boldsymbol{p} \cdot \hat{\boldsymbol{k}}}=\frac{m_{\mathrm{eff}}^{2} p_{3}}{p^{2}}\left[1-\mathcal{T}_{P}\right]$,

$\mathcal{C}^{\prime}\left(p_{0}, p_{\perp}, p_{3}\right)=m_{\mathrm{eff}}^{2} \int \frac{d \Omega}{4 \pi} \frac{1}{p_{0}-\boldsymbol{p} \cdot \hat{\boldsymbol{k}}}=\frac{m_{\mathrm{eff}}^{2}}{p_{0}} \mathcal{T}_{P}$,

where we have written the coefficients in terms of

$$
\mathcal{T}_{P}=\int \frac{d \Omega}{4 \pi} \frac{p_{0}}{p_{0}-\boldsymbol{p} \cdot \hat{\boldsymbol{k}}}
$$

which is an integral defined as the angular average over $\angle \boldsymbol{p}, \hat{\boldsymbol{k}}$. For convenience, the arguments in all those structure functions will be omitted henceforth.

\section{B. One-loop quark free energy}

In statistical field theory the partition function $Z$ can be represented as a functional determinant and by which the quark part of the free energy in one-loop order can be written as

$$
F_{q}=-N_{c} \sum_{f} \int \frac{d^{4} P}{(2 \pi)^{4}} \ln \left(\operatorname{det}\left[S_{\mathrm{eff}}^{-1}(P)\right]\right),
$$


where $P \equiv\left(p_{0}, p=|\boldsymbol{p}|\right)$ is the four momentum of the external fermion with $N_{f}$ flavor. For ideal gas of quarks the free energy reads as

$$
\begin{aligned}
F_{q}^{\text {ideal }} & =-2 N_{c} \sum_{f} \int \frac{d^{4} P}{(2 \pi)^{4}} \ln \left(-P^{2}\right) \\
& =-\frac{7 \pi^{2} T^{4}}{180} N_{c} N_{f}\left(1+\frac{120}{7} \hat{\mu}^{2}+\frac{240}{7} \hat{\mu}^{4}\right),
\end{aligned}
$$

where $\hat{\mu}=\mu / 2 \pi T$.

The quark free energy in terms of the inverse of a general quark propagator is already defined in Eq. (25). In terms of the notations introduced in Sec. IVA, we evaluate the determinant of Eq. (17) as

$$
\begin{aligned}
\operatorname{det}\left[S_{\text {eff }}^{-1}\right]= & \left(\mathcal{C}^{2} p_{0}^{2}-\mathcal{D}^{2} p^{2}+\mathcal{B}^{\prime 2}-\mathcal{C}^{\prime 2}\right)^{2} \\
& -4\left(p_{0} \mathcal{B}^{\prime} \mathcal{C}+p_{3} \mathcal{C}^{\prime} \mathcal{D}\right)^{2} \\
= & A_{0}^{2}-A_{s}^{2} .
\end{aligned}
$$

Combining Eqs. (25) and (27), the one-loop quark free energy in a weak magnetic field and HTL approximation can be written as

$$
\begin{aligned}
F_{q} & =-N_{c} \sum_{f} \int \frac{d^{4} P}{(2 \pi)^{4}} \ln \left(A_{0}^{2}-A_{s}^{2}\right) \\
& =-2 N_{c} N_{f} \int \frac{d^{4} P}{(2 \pi)^{4}} \ln \left(P^{2}\right)-N_{c} \sum_{f} \int \frac{d^{4} P}{(2 \pi)^{4}} \ln \left(\frac{A_{0}^{2}-A_{s}^{2}}{P^{4}}\right) \\
& =-\frac{7 \pi^{2} T^{4} N_{c} N_{f}}{180}\left(1+\frac{120}{7} \hat{\mu}^{2}+\frac{240}{7} \hat{\mu}^{4}\right)-N_{c} \sum_{f} \int \frac{d^{4} P}{(2 \pi)^{4}} \ln \left[\frac{\left(A_{0}+A_{s}\right)\left(A_{0}-A_{s}\right)}{P^{4}}\right] .
\end{aligned}
$$

Now the argument of the logarithm in Eq. (28) can be simplified using Eq. (27) as

$$
\begin{aligned}
\frac{\left(A_{0}+A_{s}\right)\left(A_{0}-A_{s}\right)}{P^{4}}= & 1+2\left(\frac{\mathcal{A}^{\prime}\left(\mathcal{A}^{\prime}-2\right) p_{0}^{2}-\mathcal{A}(\mathcal{A}+2) p^{2}+\mathcal{B}^{\prime 2}-\mathcal{C}^{\prime 2}}{P^{2}}\right) \\
& +\frac{\left(\mathcal{A}^{\prime}\left(\mathcal{A}^{\prime}-2\right) p_{0}^{2}-\mathcal{A}(\mathcal{A}+2) p^{2}+\mathcal{B}^{\prime 2}-\mathcal{C}^{\prime 2}\right)^{2}-4\left(\mathcal{B}^{\prime} \mathcal{C} p_{0}+\mathcal{C}^{\prime} \mathcal{D} p_{3}\right)^{2}}{P^{4}}
\end{aligned}
$$

In the high temperature limit, the logarithmic term in Eq. (28) can be expanded ${ }^{7}$ in a series of coupling constants $g$ keeping terms up to $\mathcal{O}\left(g^{4}\right)$ as

$$
\begin{aligned}
\ln \left[\frac{\left(A_{0}+A_{s}\right)\left(A_{0}-A_{s}\right)}{P^{4}}\right]= & 2\left(\frac{\mathcal{A}^{\prime 2} p_{0}^{2}-\mathcal{A}^{2} p^{2}+\mathcal{B}^{\prime 2}-\mathcal{C}^{\prime 2}-2 \mathcal{A}^{\prime} p_{0}^{2}-2 \mathcal{A} p^{2}}{P^{2}}\right) \\
& -4\left(\frac{\left(\mathcal{A}^{\prime} p_{0}^{2}+\mathcal{A} p^{2}\right)^{2}+\left(\mathcal{B}^{\prime} p_{0}+\mathcal{C}^{\prime} p_{3}\right)^{2}}{P^{4}}\right)+\mathcal{O}\left(g^{6}\right),
\end{aligned}
$$

with

$$
\begin{gathered}
\left(\mathcal{B}^{\prime 2}-\mathcal{C}^{\prime 2}\right)=m_{\text {eff }}^{4}\left[\frac{p_{3}^{2}}{p^{4}}+\frac{\mathcal{T}_{P}^{2} p_{3}^{2}}{p^{4}}-\frac{\mathcal{T}_{P}^{2}}{p_{0}^{2}}-\frac{2 \mathcal{T}_{P} p_{3}^{2}}{p^{4}}\right] \\
\left(\mathcal{B}^{\prime} p_{0}+\mathcal{C}^{\prime} p_{3}\right)^{2}=m_{\text {eff }}^{4}\left[\frac{p_{0}^{2} p_{3}^{2}}{p^{4}}\left(1+\mathcal{T}_{P}^{2}-2 \mathcal{T}_{P}\right)+\frac{\mathcal{T}_{P}^{2}}{p_{0}^{2}} p_{3}^{2}+\frac{2 p_{3}^{2}}{p^{2}}\left(\mathcal{T}_{P}-\mathcal{T}_{P}^{2}\right)\right] \\
\left(\mathcal{A}^{\prime} p_{0}^{2}+\mathcal{A} p^{2}\right)=m_{\mathrm{th}}^{2}
\end{gathered}
$$

\footnotetext{
${ }^{7}$ In Sec. VIII we will obtain the free energy without high $T$ expansion in the spirit of HTL perturbation theory and compare the result with the high $T$ expansion.
} 


$$
\left(\mathcal{A}^{\prime 2} p_{0}^{2}-\mathcal{A}^{2} p^{2}\right)=m_{\mathrm{th}}^{4}\left[\frac{\mathcal{T}_{P}^{2}}{p_{0}^{2}}-\frac{\left(1-\mathcal{T}_{P}\right)^{2}}{p^{2}}\right]
$$

So, up to $\mathcal{O}\left(g^{4}\right)$ the one-loop free energy can be written as

$$
\begin{aligned}
& F_{q}=N_{c} N_{f}\left[-\frac{7 \pi^{2} T^{4}}{180}\left(1+\frac{120}{7} \hat{\mu}^{2}+\frac{240}{7} \hat{\mu}^{4}\right)+4 m_{\mathrm{th}}^{2} \int \frac{d^{4} P}{(2 \pi)^{4}} \frac{1}{P^{2}}-m_{\mathrm{th}}^{4} \int \frac{d^{4} P}{(2 \pi)^{4}}\left[\frac{2 \mathcal{T}_{P}^{2}}{p_{0}^{2} P^{2}}-\frac{4}{P^{4}}-\frac{2}{p^{2} P^{2}}-\frac{2 \mathcal{T}_{P}^{2}}{p^{2} P^{2}}+\frac{4 \mathcal{T}_{P}}{p^{2} P^{2}}\right]\right] \\
& -N_{c} \sum_{f} m_{\text {eff }}^{4} \int \frac{d^{4} P}{(2 \pi)^{4}}\left[\frac{2}{P^{2}}\left(\frac{p_{3}^{2}}{p^{4}}+\frac{\mathcal{T}_{P}^{2} p_{3}^{2}}{p^{4}}-\frac{\mathcal{T}_{P}^{2}}{p_{0}^{2}}-\frac{2 \mathcal{T}_{P} p_{3}^{2}}{p^{4}}\right)-\frac{4}{P^{4}}\left(\frac{p_{0}^{2} p_{3}^{2}}{p^{4}}\left(1+\mathcal{T}_{P}^{2}-2 \mathcal{T}_{P}\right)\right.\right. \\
& \left.\left.+\frac{\mathcal{T}_{P}^{2}}{p_{0}^{2}} p_{3}^{2}+\frac{2 p_{3}^{2}}{p^{2}}\left(\mathcal{T}_{P}-\mathcal{T}_{P}^{2}\right)\right)\right] \\
& =N_{c} N_{f}\left[-\frac{7 \pi^{2} T^{4}}{180}\left(1+\frac{120}{7} \hat{\mu}^{2}+\frac{240}{7} \hat{\mu}^{4}\right)+4 m_{\mathrm{th}}^{2} \bigvee_{\{P\}} \frac{1}{P^{2}}\right. \\
& \left.-m_{\mathrm{th}}^{4}\left(\bigvee_{\{P\}} \frac{2 \mathcal{T}_{P}^{2}}{p_{0}^{2} P^{2}}-\bigvee_{\{P\}} \frac{4}{P^{4}}-\bigvee_{\{P\}} \frac{2}{p^{2} P^{2}}-\bigvee_{\{P\}} \frac{2 \mathcal{T}_{P}^{2}}{p^{2} P^{2}}+\bigvee_{\{P\}} \frac{4 \mathcal{T}_{P}}{p^{2} P^{2}}\right)\right] \\
& -N_{c} \sum_{f} 2 m_{\mathrm{eff}}^{4}\left(\bigvee_{\{P\}} \frac{p_{3}^{2}}{p^{4} P^{2}}+\bigvee_{\{P\}} \frac{\mathcal{T}_{P}^{2} p_{3}^{2}}{p^{4} P^{2}}-\bigvee_{\{P\}} \frac{\mathcal{T}_{P}^{2}}{p_{0}^{2} P^{2}}-\bigvee_{\{P\}} \frac{2 \mathcal{T}_{P} p_{3}^{2}}{p^{4} P^{2}}\right. \\
& \left.+2\left(\bigvee_{\{P\}} \frac{p_{3}^{2}}{p^{2} P^{4}}+\bigvee_{\{P\}} \frac{p_{3}^{2}}{p^{4} P^{2}}-\bigvee_{\{P\}} \frac{p_{3}^{2} \mathcal{T}_{P}^{2}}{p^{2} P^{4}}+\bigvee_{\{P\}} \frac{p_{3}^{2} \mathcal{T}_{P}^{2}}{p^{4} P^{2}}-\bigvee_{\{P\}} \frac{2 p_{3}^{2} \mathcal{T}_{P}}{p^{4} P^{2}}+\bigvee_{\{P\}} \frac{p_{3}^{2} \mathcal{T}_{P}^{2}}{p_{0}^{2} P^{4}}\right)\right), \\
& =N_{c} N_{f}\left[-\frac{7 \pi^{2} T^{4}}{180}\left(1+\frac{120}{7} \hat{\mu}^{2}+\frac{240}{7} \hat{\mu}^{4}\right)+\frac{m_{\mathrm{th}}^{2} T^{2}}{6}\left(1+12 \hat{\mu}^{2}\right)+4 m_{\mathrm{th}}^{4}\left[1+\frac{1-2 \Delta_{3}+\Delta_{4}^{\prime \prime}-\Delta_{3}^{\prime \prime}}{2-d}\right]{\underset{\{P\}}{P^{4}}}_{\frac{1}{P^{4}}}\right] \\
& -N_{c} \sum_{f} \frac{4 m_{\mathrm{eff}}^{4}}{2-d}\left(\Delta_{0}^{\prime}+\Delta_{4}^{\prime \prime} \Delta_{0}^{\prime}-2 \Delta_{3} \Delta_{0}^{\prime}-\Delta_{3}^{\prime \prime}\right) \sum_{\{P\}} \frac{1}{P^{4}}+N_{c} \sum_{f} 4 m_{\mathrm{eff}}^{4}\left(\left(1-\Delta_{0}^{\prime \prime}\right) \Delta_{0}^{\prime}+\left(1-\Delta_{0}^{\prime \prime}-\Delta_{10}\right) \Delta_{0}^{\prime} \frac{2}{2-d}\right. \\
& \left.+\frac{2\left(\Delta_{4}^{\prime \prime} \Delta_{0}^{\prime}-2 \Delta_{3} \Delta_{0}^{\prime}\right)}{2-d}+\frac{\Delta_{0}^{\prime \prime}}{d}-\frac{2}{d(d-2)} \Delta_{11}\right) \bigvee_{\{P\}} \frac{1}{P^{4}}
\end{aligned}
$$

where all the necessary sum integrals are provided in Appendix A. $\Delta_{i}$ 's are the $c$ integrations arising due to angular integral $\mathcal{T}_{P}$ which are computed in Appendix C. Using the expressions for various sum integrals obtained in Appendix A we can write

$$
\begin{aligned}
F_{q}= & N_{c} N_{f}\left[-\frac{7 \pi^{2} T^{4}}{180}\left(1+\frac{120}{7} \hat{\mu}^{2}+\frac{240}{7} \hat{\mu}^{4}\right)+\frac{m_{\mathrm{th}}^{2} T^{2}}{6}\left(1+12 \hat{\mu}^{2}\right)+4 m_{\mathrm{th}}^{4}\left[\left(\frac{\pi^{2}}{3}-2\right) \epsilon\right] \bigcup_{\{P\}} \frac{1}{P^{4}}\right] \\
& +N_{c} \sum_{f} m_{\mathrm{eff}}^{4}\left[\frac{2 \pi^{2}}{9}+\left(\frac{4 \zeta(3)}{3}-\frac{8}{3}-\frac{2 \pi^{2}}{27}\right) \epsilon\right] \sum_{\{P\}} \frac{1}{P^{4}}, \\
= & N_{c} N_{f}\left[-\frac{7 \pi^{2} T^{4}}{180}\left(1+\frac{120}{7} \hat{\mu}^{2}+\frac{240}{7} \hat{\mu}^{4}\right)+\frac{m_{\mathrm{th}}^{2} T^{2}}{6}\left(1+12 \hat{\mu}^{2}\right)+\frac{m_{\mathrm{th}}^{4}}{12 \pi^{2}}\left(\pi^{2}-6\right)\right] \\
& \left.+N_{c} \sum_{f} \frac{m_{\mathrm{eff}}^{4}}{16}\left(\frac{2}{9 \epsilon}+\frac{1}{27}\left(12 \ln \frac{\hat{\Lambda}}{2}-6 \aleph(z)+\frac{36 \zeta(3)}{\pi^{2}}-2-\frac{72}{\pi^{2}}\right)\right)\right],
\end{aligned}
$$




$$
\begin{aligned}
= & N_{c} N_{f}\left[-\frac{7 \pi^{2} T^{4}}{180}\left(1+\frac{120 \hat{\mu}^{2}}{7}+\frac{240 \hat{\mu}^{4}}{7}\right)+\frac{g^{2} C_{F} T^{4}}{48}\left(1+4 \hat{\mu}^{2}\right)\left(1+12 \hat{\mu}^{2}\right)\right. \\
& \left.+\frac{g^{4} C_{F}^{2} T^{4}}{768 \pi^{2}}\left(1+4 \hat{\mu}^{2}\right)^{2}\left(\pi^{2}-6\right)+\frac{g^{4} C_{F}^{2}}{27 N_{f}} M_{B}^{4}\left(12 \ln \frac{\hat{\Lambda}}{2}-6 \aleph(z)+\frac{36 \zeta(3)}{\pi^{2}}-2-\frac{72}{\pi^{2}}\right)\right]+\frac{2 N_{c} g^{4} C_{F}^{2}}{9 \epsilon} M_{B}^{4} .
\end{aligned}
$$

We note that there is no magnetic correction in the $\mathcal{O}\left[g^{2}\right]$ term in (36). Magnetic correction only appears in $\mathcal{O}\left[g^{4}\right]$ which is $\mathcal{O}\left[\left(q_{f} B\right)^{2}\right]$. We further note that the themomagnetic correction to the quark part of the free energy in a weak field has $\mathcal{O}(1 / \epsilon)$ divergence, originating due to HTL approximation. To obtain a finite contribution one needs an appropriate counterterm which will be discussed later.

\section{GLUON FREE ENERGY IN THE PRESENCE OF A MAGNETIC FIELD}

It is convenient to calculate the gluon partition function in Euclidean space. In general the QCD partition function for a gluon can be written in Euclidean space as

$$
\begin{aligned}
\mathcal{Z}_{g} & =\mathcal{Z} \mathcal{Z}^{\text {ghost }}, \\
\mathcal{Z} & =N_{\xi} \prod_{n, \boldsymbol{p}} \sqrt{\frac{(2 \pi)^{D}}{\operatorname{det} D_{\mu \nu, E}^{-1}},} \\
\mathcal{Z}^{\text {ghost }} & =\prod_{n, \boldsymbol{p}} P_{E}^{2},
\end{aligned}
$$

where the product over $n$ is for the discrete bosonic Matsubara frequencies $\left(\omega_{n}=2 \pi n \beta ; n=0,1,2, \ldots\right)$ due to Euclidean time whereas $\boldsymbol{p}$ is for the spatial momentum, $D$ is the space-time dimension of the theory, $P_{E}^{2}=\omega_{n}^{2}+p^{2}$ is the square of the four-momentum while $D_{\mu \nu, E}^{-1}$ is the inverse gauge boson propagator in Euclidean space. $N_{\xi}=1 /(2 \pi \xi)^{D / 2}$ is the normalization that originates from the introduction of the Gaussian integral at each location of position while averaging over the gauge condition function with a width $\xi$, the gauge fixing parameter.

Gluon free energy can now be written as

$$
\begin{aligned}
& F_{g}=-\left(N_{c}^{2}-1\right) \frac{T}{V} \ln \mathcal{Z}_{g}
\end{aligned}
$$

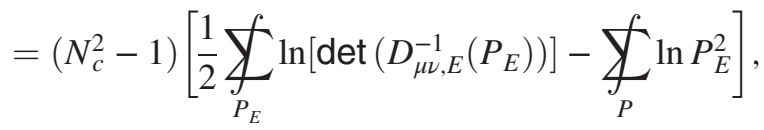

where the gauge dependence explicitly cancels due to the presence of the normalization factor $N_{\xi}$.

For an ideal case $\operatorname{det}\left(D_{\mu \nu, E}^{-1}(P)\right)=\left(P_{E}^{2}\right)^{4} / \xi$ and hence the free energy for $\left(N_{c}^{2}-1\right)$ massless spin one gluons yields as

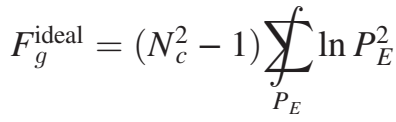

$$
\begin{aligned}
& =\left(N_{c}^{2}-1\right) \bigcup_{P} \ln \left(-P^{2}\right)=-\left(N_{c}^{2}-1\right) \frac{\pi^{2} T^{4}}{45} \text {, }
\end{aligned}
$$

where $P$ is the four-momentum in Minkowski space and can be written as $P^{2}=p_{0}^{2}-p^{2}$.

In the presence of a thermal background medium $[45,101,102]$ one can have

$$
\operatorname{det}\left(D_{\mu \nu, E}^{-1}\left(P_{E}\right)\right)=\frac{P_{E}^{2}}{\xi}\left(P_{E}^{2}+\Pi_{T}\right)^{2}\left(P_{E}^{2}+\Pi_{L}\right),
$$

with four eigenvalues, respectively $P_{E}^{2},\left(P_{E}^{2}+\Pi_{L}\right)$ and twofold degenerate $\left(P_{E}^{2}+\Pi_{T}\right)$. Here $\Pi_{T}$ and $\Pi_{L}$ are the transverse and longitudinal part of the gluon self-energy in thermal medium. Also throughout this paper we have considered spacetime dimension $D=4$ with the spatial dimension $d=3{ }^{8}$ From now on, we use Minkowski momentum $P$. Eventually the free energy becomes

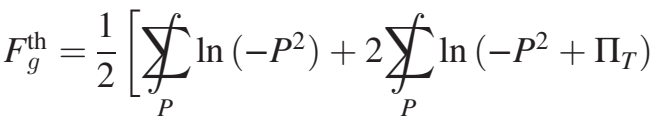

$$
\begin{aligned}
& \left.+\sum_{P} \ln \left(-P^{2}+\Pi_{L}\right)\right]-y_{P} \ln \left(-P^{2}\right), \\
& =\bigvee_{P} \ln \left(-P^{2}+\Pi_{T}\right)+\frac{1}{2} \bigvee_{P} \ln \left(1-\frac{\Pi_{L}}{P^{2}}\right) \\
& =\left(N_{c}^{2}-1\right)\left[(d-1) F_{g}^{T}+F_{g}^{L}\right],
\end{aligned}
$$

Also, $F_{g}^{T}$ and $F_{g}^{L}$ are, respectively, the transverse and longitudinal part of the gluon free energy, both of which can be computed with the help of the general structure of gauge boson self-energy evaluated in Refs. [45,101,102]. Now, in the presence of a hot magnetized medium the general structure of inverse propagator of a gauge boson is computed in Ref. [45] and reads as

\footnotetext{
${ }^{8}$ We will also use $d=3-2 \epsilon$ for dimensional regularization.
} 


$$
\begin{aligned}
\left(\mathcal{D}_{\mu \nu}\right)^{-1}= & \frac{P^{2}}{\xi} \eta_{\mu \nu}+\left(P_{m}^{2}-b\right) B_{\mu \nu}+\left(P_{m}^{2}-c\right) R_{\mu \nu} \\
& +\left(P_{m}^{2}-d\right) Q_{\mu \nu}-a N_{\mu \nu},
\end{aligned}
$$

where

$$
P_{m}^{2}=P^{2} \frac{\xi-1}{\xi}
$$

The determinant of inverse of the gauge boson propagator can be evaluated from Eq. (43) as

$$
\begin{aligned}
\operatorname{det} & \left(D_{\mu \nu, E}^{-1}(P)\right) \\
= & -\frac{P^{2}}{\xi}\left(-P^{2}+c\right)\left\{\left(-P^{2}+b\right)\left(-P^{2}+d\right)-a^{2}\right\} \\
= & -\frac{P^{2}}{\xi}\left(-P^{2}+c\right)\left(-P^{2}+\frac{b+d+\sqrt{(b-d)^{2}+4 a^{2}}}{2}\right) \\
& \times\left(-P^{2}+\frac{b+d-\sqrt{(b-d)^{2}+4 a^{2}}}{2}\right)
\end{aligned}
$$

with four eigenvalues: $-P^{2} / \xi, \quad\left(-P^{2}+c\right)$, $\left(-P^{2}+\frac{b+d+\sqrt{(b-d)^{2}+4 a^{2}}}{2}\right)$, and $\left(-P^{2}+\frac{b+d-\sqrt{(b-d)^{2}+4 a^{2}}}{2}\right)$. We note here that instead of a twofold degenerate transverse mode $\left(-P^{2}+\Pi_{T}\right)$ in thermal medium in Eq. (40), now one has two distinct transverse modes, $\left(-P^{2}+c\right)$ and $\left(-P^{2}+d\right)$. Using Eq. (45) in Eq. (38), the one-loop gluon free energy for a hot magnetized medium is given by

$$
F_{g}=\left(N_{c}^{2}-1\right)\left[\mathcal{F}_{g}^{1}+\mathcal{F}_{g}^{2}+\mathcal{F}_{g}^{3}\right]
$$

where

$$
\begin{aligned}
& \mathcal{F}_{g}^{1}=\frac{1}{2} \bigvee_{P} \ln \left(1-\frac{b+d+\sqrt{(b-d)^{2}+4 a^{2}}}{2 P^{2}}\right), \\
& \mathcal{F}_{g}^{2}=\frac{1}{2} \bigvee_{P} \ln \left(-P^{2}+c\right),
\end{aligned}
$$

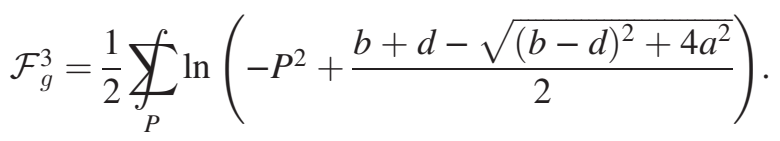

In this section, we are considering a small magnetic field approximation and we are calculating all the quantities up to $O(e B)^{2}$. Within this approximation, Eqs. (47a), (47b), $(47 \mathrm{c})$ can be approximated as

$$
\begin{aligned}
& \mathcal{F}_{g}^{1}=\frac{1}{2} \bigvee_{P} \ln \left(1-\frac{b}{P^{2}}\right), \\
& \mathcal{F}_{g}^{2}=\frac{1}{2} \bigotimes_{P} \ln \left(-P^{2}+c\right), \\
& \mathcal{F}_{g}^{3}=\frac{1}{2} \bigvee_{P} \ln \left(-P^{2}+d\right) .
\end{aligned}
$$

The various structure functions are obtained in Ref. [45] for both strong and weak field approximations. In the following subsections we discuss the QCD Debye mass and the gluon free energy in weak magnetic field approximation.

\section{A. QCD Debye mass in a magnetized hot and dense medium}

The electromagnetic Debye mass in the presence of a magnetic field was computed in $[45,55,103]$. Below we extend the calculation of Ref. [103] for QED Debye mass at finite chemical potential. Using Eq. (23) of Ref. [103] we can straightaway write down

$$
\begin{aligned}
\left.\left(m_{D}^{B}\right)^{2}\right|_{\mathrm{QED}}= & \frac{-\alpha T}{\sqrt{\pi}} e B \int_{0}^{\infty} d u \sqrt{u} \int_{-1}^{1} d v \\
& \times \sum_{l=-\infty}^{\infty} \exp \left\{-u\left(m^{2}+W_{l}^{2}\right)\right\} \\
& \times \operatorname{coth} \bar{u}\left(2 W_{l}^{2}-\frac{1}{u}\right),
\end{aligned}
$$

where $u / v, l$, and $\alpha$ represent respectively the proper time, Landau levels, and QED coupling constant with $W_{l}=(2 l+1) \pi T-i \mu$ at finite chemical potential. Now, the Poisson resummation [103] of the Landau levels is represented as

$$
\sum_{l=-\infty}^{\infty} \exp ^{-a(l-z)^{2}}=\left(\frac{\pi}{a}\right)^{1 / 2} \sum_{l=-\infty}^{\infty} \exp ^{-\pi^{2} l^{2} / a-2 i \pi z l}
$$

Taking derivative in both sides with respect to $a$, we obtain

$$
\begin{aligned}
\sum_{l=-\infty}^{\infty} e^{-a(l-z)^{2}}(l-z)^{2}= & \frac{1}{2 a}\left(\frac{\pi}{a}\right)^{1 / 2} \sum_{l=-\infty}^{\infty} \exp ^{-\pi^{2} l^{2} / a-2 i \pi z l} \\
& -\frac{\pi^{5 / 2}}{a^{5 / 2}} \sum_{l=-\infty}^{\infty} l^{2} \exp ^{-\pi^{2} l^{2} / a-2 i \pi z l} .
\end{aligned}
$$

Using Eqs. (50) and (51), we can write 


$$
\begin{aligned}
\sum_{l=-\infty}^{\infty} \exp \left(-u W_{l}^{2}\right)\left(2 W_{l}^{2}-\frac{1}{u}\right) & =\sum_{l=-\infty}^{\infty} e^{-4 u \pi^{2} T^{2}(l-i \hat{\mu}+1 / 2)^{2}}\left(8 \pi^{2} T^{2}(l-i \hat{\mu}+1 / 2)^{2}-\frac{1}{u}\right) \\
& =\sum_{l=-\infty}^{\infty} e^{-a(l-z)^{2}} \frac{1}{u}\left(2 a(l-z)^{2}-1\right), \\
& =-\frac{2 \pi^{5 / 2}}{a^{3 / 2} u} \sum_{l=-\infty}^{\infty} l^{2} \exp ^{-\pi^{2} l^{2} / a-2 i \pi z l},
\end{aligned}
$$

with $a=4 u \pi^{2} T^{2}, z=i \hat{\mu}-1 / 2, \hat{\mu}$ being $\mu / 2 \pi T$. So, Eq. (52) becomes

$$
\begin{aligned}
\sum_{l=-\infty}^{\infty} \exp \left(-u W_{l}^{2}\right)\left(2 W_{l}^{2}-\frac{1}{u}\right) & =-\frac{1}{4 \sqrt{\pi} T^{3} u^{5 / 2}} \sum_{l=-\infty}^{\infty} l^{2} \exp ^{-l^{2} / 4 u T^{2}-2 \pi \hat{\mu} l} e^{i \pi l}, \\
& =\frac{1}{4 \sqrt{\pi} T^{3} u^{5 / 2}} \sum_{l=-\infty}^{\infty}(-1)^{l+1} l^{2} \exp ^{-l^{2} / 4 u T^{2}-2 \pi \hat{\mu} l}, \\
& =\frac{1}{4 \sqrt{\pi} T^{3} u^{5 / 2}} \sum_{l=-\infty}^{\infty}(-1)^{l+1} l^{2} e^{-l^{2} / 4 u T^{2}}\left(e^{-2 \pi \hat{\mu} l}+e^{2 \pi \hat{\mu} l}\right), \\
& =\frac{1}{2 \sqrt{\pi} T^{3} u^{5 / 2}} \sum_{l=-\infty}^{\infty}(-1)^{l+1} l^{2} \cosh (2 \pi \hat{\mu} l) e^{-l^{2} / 4 u T^{2}} .
\end{aligned}
$$

Using Eq. (53) in Eq. (49), we get

$$
\left.\left(m_{D}^{B}\right)^{2}\right|_{\mathrm{QED}}=\frac{\alpha e B}{\pi T^{2}} \int_{0}^{\infty} \frac{d u}{u^{2}} \operatorname{coth}(e B u) \exp \left(-u m^{2}\right) \sum_{l=1}^{\infty}(-1)^{l+1} l^{2} \cosh (2 \pi \hat{\mu} l) \exp \left(-\frac{l^{2}}{4 u T^{2}}\right)
$$

Changing the variable from $u$ to $x=l^{2} /\left(4 u T^{2}\right)$, we get

$$
\left.\left(m_{D}^{B}\right)^{2}\right|_{\mathrm{QED}}=\frac{e^{2} e B}{\pi^{2} T^{2}} \int_{0}^{\infty} e^{-x} d x \sum_{l=1}^{\infty}(-1)^{l+1} \operatorname{coth}\left(\frac{e B l^{2}}{4 x T^{2}}\right) \exp \left(-\frac{m^{2} l^{2}}{4 x T^{2}}\right)
$$

Generalizing this to QCD we obtain the expression for the modified QCD Debye mass at finite chemical potential and an arbitrary magnetic field as

$$
\left(m_{D}^{B}\right)^{2}=\frac{g^{2} N_{c} T^{2}}{3}+\sum_{f} \frac{g^{2} q_{f} B}{2 \pi^{2}} \int_{0}^{\infty} e^{-x} d x \sum_{l=1}^{\infty}(-1)^{l+1} \cosh (2 l \pi \hat{\mu}) \operatorname{coth}\left(\frac{q_{f} B l^{2}}{4 x T^{2}}\right) \exp \left(-\frac{m_{f}^{2} l^{2}}{4 x T^{2}}\right),
$$

where the first term in Eq. (56) is due to the pure gluonic contributions, which was not present in the case of QED. The second term comes from quark loop contribution which is obtained by replacing $m$ with $m_{f}$ and $e$ with $g$. Also a quarkflavor sum with QCD factor as $\frac{1}{2} \sum_{f}$ is considered.

Now, in the strong magnetic field limit $\left(m_{\mathrm{th}}^{2} \sim g^{2} T^{2} \leq T^{2} \leq q_{f} B\right)$, i.e., in LLL, neglecting the current quark mass $m_{f}$, from Eq. (56) we can straightaway reach a simplified expression ${ }^{9}$ given as

$$
\left(m_{D}^{s}\right)^{2}=\frac{g^{2} N_{c} T^{2}}{3}+\sum_{f} \frac{g^{2} q_{f} B}{4 \pi^{2}} .
$$

\footnotetext{
${ }^{9}$ Our fermionic part of the Debye mass is different from Ref. [52] by a factor of 2 which was somehow overlooked by the authors of Ref. [52] in Matsurbara sum. We also find the same mismatch with Ref. [104].
} 

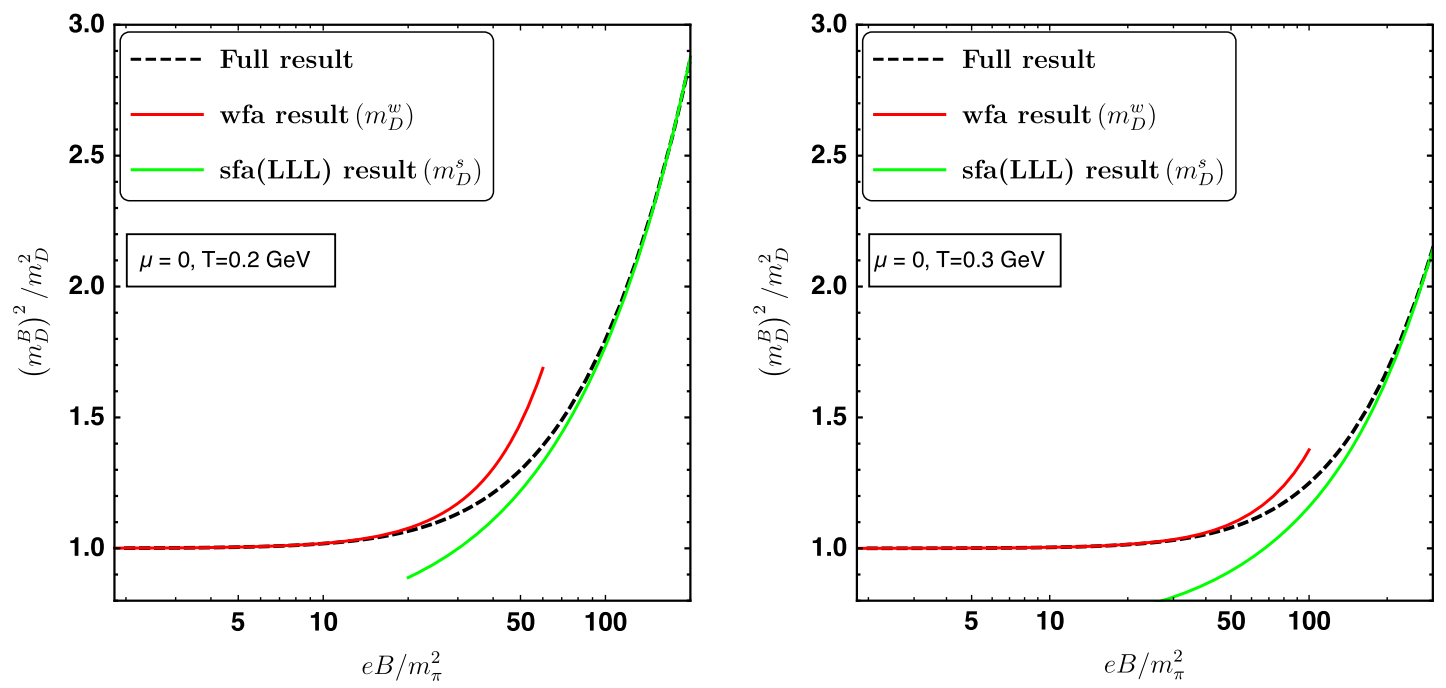

FIG. 3. Comparison of the scaled one-loop Debye masses in Eqs. (56), (57), and (58) varying with scaled magnetic field for $N_{f}=3$, $\mu=0$. Left: $T=200 \mathrm{MeV}$. Right: $T=300 \mathrm{MeV}$.

We were able to get the same expression for Debye mass in the strong magnetic field limit as in Eq. (57) when we calculate the gluon polarization tensor using a quark propagator in strong field approximation and take the static limit of the zero-zero component of that tensor [45].

In the weak field approximation $\left(T^{2}>m_{\mathrm{th}}^{2}>q_{f} B\right)$, the square of Debye mass can be obtained from Eq. (56) by expanding coth $\left(q_{f} B l^{2} / 4 x T^{2}\right)$ as

$$
\begin{aligned}
\left(m_{D}^{w}\right)^{2} \simeq & \frac{g^{2} T^{2}}{3}\left[\left(N_{c}+\frac{N_{f}}{2}\right)+6 N_{f} \hat{\mu}^{2}\right] \\
& +\sum_{f} \frac{g^{2}\left(q_{f} B\right)^{2}}{12 \pi^{2} T^{2}} \sum_{l=1}^{\infty}(-1)^{l+1} l^{2} \cosh (2 l \pi \hat{\mu}) K_{0}\left(\frac{m_{f} l}{T}\right) \\
& +\mathcal{O}\left[\left(q_{f} B\right)^{4}\right] \\
& =m_{D}^{2}+\delta m_{D}^{2}
\end{aligned}
$$

where $m_{D}$ can be identified as the QCD Debye mass in a hot and dense medium in the absence of any external magnetic field and $K_{n}(z)$ represents the modified Bessel function of the second kind. This second term is the thermomagnetic correction due to the presence of the weak external magnetic field. Note that Eq. (58) is valid only when $\mu \leq m_{f}$, as the infinite sum over $l$ diverges at $\mu>m_{f}$.

In Fig. 3 the full expression in Eq. (56), the strong field expression in Eq. (57), and the weak field expression in Eq. (58) scaled with $m_{D}$ are displayed as a function of the magnetic field scaled with squared pion mass. In the strong field limit, (e.g., for $T=200 \mathrm{MeV},|e B| / m_{\pi}^{2} \geq 10$ ) the weak field result (red colored curve) starts to deviate from the full result (dashed line). However, there is no difference between the two in the limit $|e B| / m_{\pi}^{2}<10$, so it defines the domain of a weak field for $T=200 \mathrm{MeV}$ and is indeed a good approximation to work with Eq. (58) in the weak field limit at that temperature. On the other hand, the LLL result (green colored line) agrees with the full when, e.g., for $\left.T=200 \mathrm{MeV},|e B| / m_{\pi}^{2} \geq 70\right) \mathrm{MeV}$. The right panel is for $T=300 \mathrm{MeV}$ which shows the same behavior. However, these two plots indicate that the domain of applicability for strong $\left(|e B|>T^{2}\right)$ and weak $\left(|e B|<T^{2}\right)$ fields changes quantitatively with the change in temperature. In between the weak and strong field domain in principle one should work with a full expression which is indeed a very involved and difficult task. However, presently we confine ourselves in the weak magnetic field limit.

\section{B. One-loop gluon free energy in weakly magnetized hot medium}

The form factors for the gluonic self-energy in a weakly magnetized medium can be expressed by breaking into thermal and thermomagnetic parts each, as

$$
\begin{aligned}
& b(T, \mu, B)=b_{0}(T, \mu)+b_{2}(T, \mu, B), \\
& c(T, \mu, B)=c_{0}(T, \mu)+c_{2}(T, \mu, B), \\
& d(T, \mu, B)=d_{0}(T, \mu)+d_{2}(T, \mu, B),
\end{aligned}
$$

with 


$$
\begin{aligned}
b_{0}= & \frac{m_{D}^{2}}{\bar{u}^{2}}\left(1-\mathcal{T}_{P}\right), \\
c_{0}= & d_{0}=\frac{m_{D}^{2}}{2 p^{2}}\left[p_{0}^{2}-P^{2} \mathcal{T}_{P}\right], \\
b_{2}= & \frac{\delta m_{D}^{2}}{\bar{u}^{2}}+\sum_{f} \frac{g^{2}\left(q_{f} B\right)^{2}}{\bar{u}^{2} \pi^{2}}\left[\left(g_{k}+\frac{\pi m_{f}-4 T}{32 m_{f}^{2} T}\right)\left(A_{0}-A_{2}\right)+\left(f_{k}+\frac{8 T-\pi m_{f}}{128 m_{f}^{2} T}\right)\left(\frac{5}{3} A_{0}-A_{2}\right)\right], \\
c_{2}= & -\sum_{f} \frac{4 g^{2}\left(q_{f} B\right)^{2}}{3 \pi^{2}} g_{k}+\sum_{f} \frac{g^{2}\left(q_{f} B\right)^{2}}{2 \pi^{2}}\left(g_{k}+\frac{\pi m_{f}-4 T}{32 m_{f}^{2} T}\right) \times\left[-\frac{7}{3} \frac{p_{0}^{2}}{p_{1}^{2}}+\left(2+\frac{3 p_{0}^{2}}{2 p_{1}^{2}}\right) A_{0}+\left(\frac{3}{2}+\frac{5 p_{0}^{2}}{2 p_{1}^{2}}+\frac{3 p_{3}^{2}}{2 p_{1}^{2}}\right) A_{2}\right. \\
& \left.-\frac{3 p_{0} p_{3}}{p_{1}^{2}} A_{1}-\frac{5}{2}\left(1-\frac{p_{3}^{2}}{p_{1}^{2}}\right) A_{4}-\frac{5 p_{0} p_{3}}{p_{1}^{2}} A_{3}\right], \\
d_{2}= & -\sum_{f} \frac{g^{2}\left(q_{f} B\right)^{2}}{\pi^{2}} \frac{p^{2}}{p_{1}^{2}}\left[g_{k} \frac{p_{0}^{2} p_{3}^{2}}{3 p^{4}}+\left(g_{k}+\frac{\pi m_{f}-4 T}{32 m_{f}^{2} T}\right)\left\{\frac{A_{0}}{4}-\left(\frac{3}{2}+\frac{p_{0}^{2} p_{3}^{2}}{p^{4}}\right) A_{2}+\frac{5 A_{4}}{4}\right\}-\frac{14}{3} f_{k} \frac{p_{0}^{2} p_{3}^{2}}{p^{4}}\right. \\
& \left.+\left(f_{k}+\frac{8 T-\pi m_{f}}{128 m_{f}^{2} T}\right) \frac{p_{0}^{2} p_{3}^{2}}{p^{4}}\left(5 A_{0}-A_{2}\right)\right]+\sum_{f} \frac{g^{2}\left(q_{f} B\right)^{2}}{6 \pi^{2} m_{f} T} \frac{\left(\frac{3 A_{1}}{2}-A_{3}\right)}{\left(1+\cosh _{\frac{m_{f}}{T}}\right)} \frac{p_{0} p_{3}}{p_{1}^{2}},
\end{aligned}
$$

where $f_{k}, g_{k}$, and $A_{n}=\int \frac{d \Omega}{4 \pi} \frac{c^{n} p_{0}}{p_{0}-\boldsymbol{p} \cdot \hat{\boldsymbol{k}}}$ are calculated in paper-I [45] with $A_{0} \equiv \mathcal{T}_{P}{ }^{10}$

Therefore, one can write the longitudinal and transverse parts of the gluonic free energy in a weakly magnetized medium respectively as

$$
\mathcal{F}_{g}^{1}=\frac{1}{2} y_{P} \ln \left(1-\frac{b}{P^{2}}\right)=\underbrace{-\frac{1}{2} \bigvee_{P}\left(\frac{b_{0}}{P^{2}}+\frac{b_{0}^{2}}{2 P^{4}}\right)}_{\text {thermal part: } \mathcal{F}_{g}^{1 T}} \underbrace{-\frac{1}{2} \bigvee_{P}\left(\frac{b_{2}}{P^{2}}+\frac{b_{0} b_{2}}{P^{4}}\right)}_{\text {thermomagnetic part: } \mathcal{F}_{g}^{1 B}}-\cdots=\mathcal{F}_{g}^{1 T}+\mathcal{F}_{g}^{1 B}
$$

and

$$
\begin{aligned}
\mathcal{F}_{g}^{2}+\mathcal{F}_{g}^{3} & =\left[\bigcup_{P} \ln \left(-P^{2}\right)+\frac{1}{2} \bigvee_{P} \ln \left(1-\frac{c}{P^{2}}\right)+\frac{1}{2} \bigvee_{P} \ln \left(1-\frac{d}{P^{2}}\right)\right] \\
& =\underbrace{-\frac{\pi^{2} T^{4}}{45}-\bigvee_{P}\left(\frac{c_{0}}{P^{2}}+\frac{c_{0}^{2}}{2 P^{4}}\right)}_{\text {thermal part: } \mathcal{F}_{g}^{2 T}+\mathcal{F}_{g}^{3 T}} \underbrace{-\frac{1}{2} \bigvee_{P}\left(c_{2}+d_{2}\right)\left(\frac{1}{P^{2}}+\frac{c_{0}}{P^{4}}\right)}_{\text {thermomagnetic part: } \mathcal{F}_{g}^{2 B}+\mathcal{F}_{g}^{3 B}} \\
& =\left(\mathcal{F}_{g}^{2 T}+\mathcal{F}_{g}^{3 T}\right)+\left(\mathcal{F}_{g}^{2 B}+\mathcal{F}_{g}^{3 B}\right),
\end{aligned}
$$

where we have kept terms up to $\mathcal{O}\left(q_{f} B\right)^{2}$ in high $T$ expansion in the same spirit as was done for the quark case.

Using Eqs. (60) and (61) and sum integrals from Appendix B, the total thermal part (i.e., magnetic field independent terms) can straightaway be written as

$$
\begin{aligned}
& \mathcal{F}_{g}^{1 T}+\mathcal{F}_{g}^{2 T}+\mathcal{F}_{g}^{3 T}=-\frac{\pi^{2} T^{4}}{45}-\frac{1}{2} \bigvee_{P}\left(\frac{b_{0}}{P^{2}}+\frac{b_{0}^{2}}{2 P^{4}}\right)-\bigvee_{P}\left(\frac{c_{0}}{P^{2}}+\frac{c_{0}^{2}}{2 P^{4}}\right) \\
& =-\frac{\pi^{2} T^{4}}{45}-\frac{m_{D}^{2}}{2} y_{P} \frac{1}{P^{2}}-\frac{m_{D}^{4}}{8} \bigvee_{P}\left[\frac{1}{P^{4}}+\frac{2}{p^{2} P^{2}}-\frac{6 \mathcal{T}_{p}}{p^{4}}-\frac{2 \mathcal{T}_{p}}{p^{2} P^{2}}+\frac{3 \mathcal{T}_{p}^{2}}{p^{4}}\right] \\
& =-\frac{\pi^{2} T^{4}}{45}+\frac{m_{D}^{2} T^{2}}{24}-\frac{m_{D}^{4}}{128 \pi^{2}}\left(\frac{\Lambda}{4 \pi T}\right)^{2 \epsilon}\left[\frac{1}{\epsilon}+2 \gamma_{E}+\frac{2 \pi^{2}}{3}-7\right] .
\end{aligned}
$$

In the following two subsubsections we evaluate the thermomagnetic parts of the gluon free energy.

\footnotetext{
${ }^{10}$ We hereby note that although in paper-I [45] $f_{k}$ and $g_{k}$ are defined with zero chemical potential, it has been checked that within the small $m_{f}$ approximation (which we are going to use by the virtue of HTLpt), the expressions for $f_{k}$ and $g_{k}$ do not change with the inclusion of a finite chemical potential [see Eq. (64)].
} 


\section{Longitudinal part: $\mathcal{F}_{g}^{1 B}$}

The thermomagnetic contribution of the longitudinal part can subsequently be expressed from Eqs. (60) and (61) as

$$
\begin{aligned}
& \mathcal{F}_{g}^{1 B}=-\frac{1}{2} \bigvee_{P}\left(\frac{b_{2}}{P^{2}}+\frac{b_{0} b_{2}}{P^{4}}\right)=\frac{m_{D}^{2} \delta m_{D}^{2}}{2} \bigcup_{P} \frac{\mathcal{T}_{P}}{p^{4}}+\sum_{f} \frac{g^{2}\left(q_{f} B\right)^{2}}{2 \pi^{2}} \\
& \times\left[\left(g_{k}+\frac{\pi m_{f}-4 T}{32 m_{f}^{2} T}\right)\left(\bigvee_{P} \frac{\mathcal{T}_{P}}{p^{2}}-\bigvee_{P} \frac{A_{2}}{p^{2}}-m_{D}^{2} \bigvee_{P} \frac{\left(1-\mathcal{T}_{P}\right)\left(\mathcal{T}_{P}-A_{2}\right)}{p^{4}}\right)\right. \\
& \left.+\left(f_{k}+\frac{8 T-\pi m_{f}}{128 m_{f}^{2} T}\right)\left(\frac{5}{3} \bigvee_{P} \frac{\mathcal{T}_{P}}{p^{2}}-\bigvee_{P} \frac{A_{2}}{p^{2}}-m_{D}^{2} \bigvee_{P} \frac{\left(1-\mathcal{T}_{P}\right)\left(\frac{5}{3} \mathcal{T}_{P}-A_{2}\right)}{p^{4}}\right)\right] \\
& =-\frac{m_{D}^{2} \delta m_{D}^{2}}{(4 \pi)^{2}}\left(\frac{\Lambda e^{\gamma_{E}}}{4 \pi T}\right)^{2 \epsilon}\left(\frac{1}{2 \epsilon}+\ln 2\right)-\sum_{f} \frac{g^{2}\left(q_{f} B\right)^{2}}{2 \pi^{2}}\left(\frac{\Lambda}{4 \pi T}\right)^{2 \epsilon}\left[\left(g_{k}+\frac{\pi m_{f}-4 T}{32 m_{f}^{2} T}\right)\right. \\
& \times\left(\frac{T^{2}}{72}\left[\frac{2}{\epsilon}+11.046\right]-\frac{2 m_{D}^{2} e^{2 \gamma_{E} \epsilon}}{9(4 \pi)^{2}}\left[\frac{1-4 \ln 2}{\epsilon}-5.326\right]\right)+\left(f_{k}+\frac{8 T-\pi m_{f}}{128 m_{f}^{2} T}\right) \\
& \left.\times\left(\frac{T^{2}}{72}\left[\frac{4}{\epsilon}+21.759\right]-\frac{2 m_{D}^{2} e^{2 \gamma_{E} \epsilon}}{9(4 \pi)^{2}}\left[\frac{2(1-4 \ln 2)}{\epsilon}-10.589\right]\right)\right] .
\end{aligned}
$$

By virtue of HTLpt, we now make a small $m_{f}$ approximation within which

$$
g_{k} \approx-2 f_{k} \approx \frac{1}{8 m_{f}^{2}}
$$

and subsequently Eq. (63) becomes

$$
\begin{aligned}
\mathcal{F}_{g}^{1 B}= & -\frac{m_{D}^{2} \delta m_{D}^{2}}{(4 \pi)^{2}}\left(\frac{\Lambda}{4 \pi T}\right)^{2 \epsilon}\left(\frac{1}{2 \epsilon}+\ln 2+\gamma_{E}\right)-\sum_{f} \frac{g^{2}\left(q_{f} B\right)^{2}}{(12 \pi)^{2}} \frac{\pi T}{32 m_{f}}\left(\frac{\Lambda}{4 \pi T}\right)^{2 \epsilon} \\
& \times\left\{\left(\frac{1}{\epsilon}+5.606\right)+\frac{3 \hat{m}_{D}^{2}}{4}\left(\frac{8(4 \ln 2-1)}{3 \epsilon}+19.7467\right)\right\},
\end{aligned}
$$

with divergence of $\mathcal{O}(1 / \epsilon)$.

\section{Transverse part: $\mathcal{F}_{g}^{2 B}$ and $\mathcal{F}_{g}^{3 B}$}

From Eq. (61) it is evident that to evaluate a thermomagnetic contribution for the transverse part of the gluonic free energy in a weakly magnetized medium one needs to compute the following sum integrals:

$$
\begin{aligned}
& \sum_{P} \frac{c_{2}+d_{2}}{P^{2}}=\sum_{f} \frac{g^{2}\left(q_{f} B\right)^{2}}{\pi^{2}}[\left(14 f_{k}-g_{k}\right) \bigotimes_{P} \frac{p_{0}^{2} p_{3}^{2}}{3 p_{1}^{2} p^{2} P^{2}}-\frac{4}{3} g_{k} \bigcup_{P} \frac{1}{P^{2}}+\left(g_{k}+\frac{\pi m_{f}-4 T}{32 m_{f}^{2} T}\right) \underbrace{}_{P} \\
& \times\left\{-\frac{7}{6} \frac{p_{0}^{2}}{p_{1}^{2} P^{2}}+\frac{A_{0}}{P^{2}}\left(1+\frac{3 p_{0}^{2}-p^{2}}{4 p_{1}^{2}}\right)+\frac{A_{2}}{P^{2}}\left(\frac{5 p_{0}^{2}+9 p^{2}}{4 p_{1}^{2}}+\frac{p_{0}^{2} p_{3}^{2}}{p_{1}^{2} p^{2}}\right)-\frac{5 A_{4}}{2 P^{2}}-\frac{p_{0} p_{3}}{2 p_{1}^{2} P^{2}}\left(3 A_{1}+5 A_{3}\right)\right\} \\
& \left.-\left(f_{k}+\frac{8 T-\pi m_{f}}{128 m_{f}^{2} T}\right) \bigcup_{P} \frac{p_{0}^{2} p_{3}^{2}\left(5 A_{0}-A_{2}\right)}{p_{1}^{2} p^{2} P^{2}}+\frac{\left(1+\cosh \frac{m_{f}}{T}\right)^{-1}}{6 m_{f} T} \bigcup_{P} \frac{\left(\frac{3 A_{1}}{2}-A_{3}\right) p_{0} p_{3}}{p_{1}^{2} P^{2}}\right],
\end{aligned}
$$

and 


$$
\begin{aligned}
& \underbrace{}_{P} \frac{c_{0}\left(c_{2}+d_{2}\right)}{P^{4}}=\sum_{f} \frac{g^{2}\left(q_{f} B\right)^{2} m_{D}^{2}}{2 \pi^{2}}\left[\left(14 f_{k}-g_{k}\right) \bigotimes_{P} \frac{p_{0}^{2} p_{3}^{2}\left(p_{0}^{2}-A_{0} P^{2}\right)}{3 p_{1}^{2} p^{4} P^{4}}-\frac{4}{3} g_{k}\right\}_{P} \frac{p_{0}^{2}-A_{0} P^{2}}{p^{2} P^{4}} \\
& +\left(g_{k}+\frac{\pi m_{f}-4 T}{32 m_{f}^{2} T}\right) \bigotimes_{P}\left(\frac{p_{0}^{2}-A_{0} P^{2}}{p^{2} P^{2}}\right)\left\{-\frac{7}{6} \frac{p_{0}^{2}}{p_{1}^{2} P^{2}}+\frac{A_{0}}{P^{2}}\left(1+\frac{3 p_{0}^{2}-p^{2}}{4 p_{1}^{2}}\right)\right. \\
& \left.+\frac{A_{2}}{P^{2}}\left(\frac{5 p_{0}^{2}+9 p^{2}}{4 p_{1}^{2}}+\frac{p_{0}^{2} p_{3}^{2}}{p_{1}^{2} p^{2}}\right)-\frac{5 A_{4}}{2 P^{2}}-\frac{p_{0} p_{3}}{2 p_{1}^{2} P^{2}}\left(3 A_{1}+5 A_{3}\right)\right\}-\left(f_{k}+\frac{8 T-\pi m_{f}}{128 m_{f}^{2} T}\right) \\
& \left.\times \bigvee_{P} \frac{p_{0}^{2} p_{3}^{2}\left(5 A_{0}-A_{2}\right)\left(p_{0}^{2}-A_{0} P^{2}\right)}{p_{1}^{2} p^{4} P^{4}}+\frac{\left(1+\cosh \frac{m_{f}}{T}\right)^{-1}}{6 m_{f} T} \bigcup_{P} \frac{\left(\frac{3 A_{1}}{2}-A_{3}\right) p_{0} p_{3}\left(p_{0}^{2}-A_{0} P^{2}\right)}{p_{1}^{2} p^{2} P^{4}}\right] .
\end{aligned}
$$

Hence, using various specific HTL sum integrals listed in Appendix B, the master sum integrals listed in Appendix B 2 b along with angular integrations listed in Appendix C, one obtains

$$
\begin{aligned}
& \mathcal{F}_{g}^{2 B}+\mathcal{F}_{g}^{3 B}=-\frac{1}{2} \bigvee_{P}\left(c_{2}+d_{2}\right)\left(\frac{1}{P^{2}}+\frac{c_{0}}{P^{4}}\right) \\
& =-\sum_{f} \frac{g^{2}\left(q_{f} B\right)^{2} T^{2}}{144 \pi^{2}}\left(\frac{\Lambda}{4 \pi T}\right)^{2 \epsilon}\left[( 1 4 f _ { k } - g _ { k } ) \left\{\left(\frac{1}{\epsilon}+24 \ln G\right)+3 \hat{m}_{D}^{2}\left[\frac{1-\ln 2}{\epsilon^{2}}+\frac{1}{\epsilon}\left(4-\frac{\pi^{2}}{6}-\ln ^{2}(2)\right.\right.\right.\right. \\
& \left.\left.\left.-2 \gamma_{E}(\ln 2-1)-\ln 2\right)+4.38\right]\right\}+g_{k}\left\{8+3 \hat{m}_{D}^{2}\left[\frac{1}{\epsilon}(4-4 \ln 4)-2.79\right]\right\}+\left(g_{k}+\frac{\pi m_{f}-4 T}{32 m_{f}^{2} T}\right) \\
& \times\left\{\frac{3}{8 \epsilon^{2}}+\frac{1}{4 \epsilon}(36 \ln G+2+15 \ln 2)+20.83+3 \hat{m}_{D}^{2}\left[\frac{1}{\epsilon^{2}}\left(-\frac{319}{20}+\pi^{2}+\frac{89 \ln 2}{10}\right)+\frac{1}{600 \epsilon}(3600 \zeta(3)\right.\right. \\
& \left.\left.\left.-37658+2900 \pi^{2}+5340 \ln ^{2}(2)+4736 \ln 2+30 \gamma_{E}\left(-638+40 \pi^{2}+356 \ln 2\right)\right)+7.18\right]\right\} \\
& -\left(f_{k}+\frac{8 T-\pi m_{f}}{128 m_{f}^{2} T}\right)\left\{\frac{3}{2}\left(\frac{1+8 \ln 2}{\epsilon}+45.68\right)+3 \hat{m}_{D}^{2}\left[\frac{1}{10 \epsilon^{2}}\left(29+10 \pi^{2}-128 \ln 2\right)+\frac{1}{25 \epsilon}(150 \zeta(3)\right.\right. \\
& \left.\left.\left.+564+\frac{125 \pi^{2}}{6}+5 \gamma_{E}\left(29+10 \pi^{2}-128 \ln 2\right)-4 \ln 2(147+80 \ln 2)\right)+58.01\right]\right\} \\
& +\frac{\left(1+\cosh \frac{m_{f}}{T}\right)^{-1}}{6 m_{f} T}\left\{\frac{3 \ln 2-4}{2 \epsilon}-3.92+3 \hat{m}_{D}^{2} \times\left[\frac{1}{40 \epsilon^{2}}\left(11+5 \pi^{2}-92 \ln 2\right)+\frac{1}{600 \epsilon}(450 \zeta(3)+4671\right.\right. \\
& \left.\left.\left.\left.-200 \pi^{2}-1380 \ln ^{2}(2)-4032 \ln 2+30 \gamma_{E}\left(11+5 \pi^{2}-92 \ln 2\right)\right)-1.86\right]\right\}\right] \text {. }
\end{aligned}
$$

Similar to the longitudinal part, using small $m_{f}$ approximations in Eq. (68), we get

$$
\begin{aligned}
\mathcal{F}_{g}^{2 B}+\mathcal{F}_{g}^{3 B}= & \sum_{f} \frac{g^{2}\left(q_{f} B\right)^{2}}{(12 \pi)^{2}} \frac{T^{2}}{m_{f}^{2}}\left(\frac{\Lambda}{4 \pi T}\right)^{2 \epsilon} \times\left[\frac{1}{\epsilon}+4.97+3 \hat{m}_{D}^{2}\left\{\frac{1-\ln 2}{\epsilon^{2}}+\frac{1}{\epsilon}\left(\frac{7}{2}-\frac{\pi^{2}}{6}-\ln ^{2}(2)-2 \gamma_{E}(\ln 2-1)\right)+4.73\right\}\right] \\
& -\sum_{f} \frac{g^{2}\left(q_{f} B\right)^{2}}{(12 \pi)^{2}} \frac{\pi T}{32 m_{f}}\left(\frac{\Lambda}{4 \pi T}\right)^{2 \epsilon}\left\{\frac{3}{8 \epsilon^{2}}+\frac{1}{\epsilon}\left(\frac{13}{8}+\frac{3}{4} \frac{\zeta^{\prime}(-1)}{\zeta(-1)}+\frac{27}{4} \ln 2\right)+37.96\right. \\
& +\frac{3}{4} \hat{m}_{D}^{2}\left[\frac{1}{\epsilon^{2}}\left(5 \pi^{2}-\frac{609}{10}+\frac{114 \ln 2}{5}\right)+\frac{1}{\epsilon}\left(30 \zeta(3)-\frac{17137}{75}+\frac{121}{6} \pi^{2}+\frac{114}{5} \ln ^{2}(2)\right.\right. \\
& \left.\left.\left.+\frac{604}{75} \ln 2+\gamma_{E}\left(10 \pi^{2}-\frac{609}{5}+\frac{228}{5} \ln 2\right)\right)+86.73\right]\right\}-\sum_{f} \frac{g^{2}\left(q_{f} B\right)^{2}}{(12 \pi)^{2}} \frac{T}{12 m_{f}}\left\{\frac{3 \ln 2-4}{2 \epsilon}\right.
\end{aligned}
$$




$$
\begin{aligned}
& -3.92+3 \hat{m}_{D}^{2}\left[\frac{1}{40 \epsilon^{2}}\left(11+5 \pi^{2}-92 \ln 2\right)+\frac{1}{600 \epsilon}\left(450 \zeta(3)+4671-200 \pi^{2}-1380 \ln ^{2}(2)\right.\right. \\
& \left.\left.\left.-4032 \ln 2+30 \gamma_{E}\left(11+5 \pi^{2}-92 \ln 2\right)\right)-1.86\right]\right\} .
\end{aligned}
$$

We note that the gluon free energy also contains a thermomagnetic correction starting from $\mathcal{O}\left[\left|q_{f} B\right|^{2}\right]$ as the form factors start with $\mathcal{O}\left[\left|q_{f} B\right|^{2}\right]$.

Due to high temperature expansion within the HTL approximation, there also appears a soft contribution [78] as

$$
\mathcal{F}_{g}^{\text {soft }}=-\frac{1}{12 \pi}\left(m_{D}^{w}\right)^{3} T
$$

where $m_{D}^{w}$ is given in Eq. (58). This contains contributions from both the thermal and thermomagnetic parts.

\section{TOTAL FREE ENERGY AND PRESSURE IN WEAK FIELD APPROXIMATION}

\section{A. Free energy}

Finally, the total one-loop free energy of a weakly magnetized hot medium as written in Eq. (1) reads as

$$
F=F_{q}+F_{g}+F_{0}+\Delta \mathcal{E}_{0}
$$

where the quark part of the free energy $F_{q}$ has both HTL (viz., magnetic field independent) part as well as the thermomagnetic correction as obtained in Eq. (36). Similarly, the gluonic part has also an HTL part $\left(F_{g}^{\mathrm{HTL}}\right)$ plus the thermomagnetic correction $\left(F_{g}^{B}\right)$. Thus the total gluonic free energy $F_{g}$ can be written from Eqs. (62), (63), (68), (70) as

$$
\begin{aligned}
& F_{g}=d_{A}\left[\mathcal{F}_{g}^{1 T}+\mathcal{F}_{g}^{2 T}+\mathcal{F}_{g}^{3 T}+\mathcal{F}_{g}^{\text {soft }}+\mathcal{F}_{g}^{1 B}+\mathcal{F}_{g}^{2 B}+\mathcal{F}_{g}^{3 B}\right]+\Delta \mathcal{E}_{0} \\
& =-\frac{d_{A} \pi^{2} T^{4}}{45}\left[1-\frac{15}{2} \hat{m}_{D}^{2}+30\left(\hat{m}_{D}^{w}\right)^{3}+\frac{45}{8} \hat{m}_{D}^{4}\left(2 \ln \frac{\hat{\Lambda}}{2}-7+2 \gamma_{E}+\frac{2 \pi^{2}}{3}\right)\right] \\
& +d_{A}\left[-\frac{m_{D}^{2} \delta m_{D}^{2}}{(4 \pi)^{2}}\left(\frac{\Lambda}{4 \pi T}\right)^{2 \epsilon}\left(\frac{1}{2 \epsilon}+\ln 2+\gamma_{E}\right)+\sum_{f} \frac{g^{2}\left(q_{f} B\right)^{2}}{(12 \pi)^{2}} \frac{T^{2}}{m_{f}^{2}}\left(\frac{\Lambda}{4 \pi T}\right)^{2 \epsilon}\left[\frac{1}{\epsilon}+4.97\right.\right. \\
& \left.+3 \hat{m}_{D}^{2}\left\{\frac{1-\ln 2}{\epsilon^{2}}+\frac{1}{\epsilon}\left(\frac{7}{2}-\frac{\pi^{2}}{6}-\ln ^{2}(2)-2 \gamma_{E}(\ln 2-1)\right)+4.73\right\}\right] \\
& -\sum_{f} \frac{g^{2}\left(q_{f} B\right)^{2}}{(12 \pi)^{2}} \frac{\pi T}{32 m_{f}}\left(\frac{\Lambda}{4 \pi T}\right)^{2 \epsilon}\left[\left\{\frac{3}{8 \epsilon^{2}}+\frac{1}{\epsilon}\left(\frac{21}{8}+\frac{3}{4} \frac{\zeta^{\prime}(-1)}{\zeta(-1)}+\frac{27}{4} \ln 2\right)+43.566\right.\right. \\
& +\frac{3}{4} \hat{m}_{D}^{2}\left[\frac{1}{\epsilon^{2}}\left(5 \pi^{2}-\frac{609}{10}+\frac{114 \ln 2}{5}\right)+\frac{1}{\epsilon}\left(30 \zeta(3)-\frac{5779}{75}+\frac{121}{6} \pi^{2}+\frac{114}{5} \ln ^{2}(2)\right.\right. \\
& \left.\left.\left.+\frac{468}{25} \ln 2+\gamma_{E}\left(10 \pi^{2}-\frac{609}{5}+\frac{228}{5} \ln 2\right)\right)+106.477\right]\right\}+\frac{8}{3 \pi}\left\{\frac{3 \ln 2-4}{2 \epsilon}-3.92\right. \\
& +3 \hat{m}_{D}^{2}\left[\frac{1}{40 \epsilon^{2}}\left(11+5 \pi^{2}-92 \ln 2\right)+\frac{1}{\epsilon}\left(\frac{3}{4} \zeta(3)+\frac{1557}{200}-\frac{\pi^{2}}{3}-\frac{23}{10} \ln ^{2}(2)\right.\right. \\
& \left.\left.\left.\left.\left.-\frac{168}{25} \ln 2+\gamma_{E}\left(\frac{11}{20}+\frac{\pi^{2}}{4}-\frac{23}{5} \ln 2\right)\right)-1.86\right]\right\}\right]\right]
\end{aligned}
$$

where we have also used the HTL counterterm [80] as given in Eq. (2), $d_{A}=N_{c}^{2}-1, \hat{m}_{D}=m_{D} / 2 \pi T$ and $\hat{m}_{D}^{w}=m_{D}^{w} / 2 \pi T$.

The magnetic field dependent gluonic free energy has both $\mathcal{O}(1 / \epsilon)$ (UV) and $\mathcal{O}\left(1 / \epsilon^{2}\right.$ ) (both colinear and UV) divergences. Now, the external magnetic field $B$ dependent divergences present in Eqs. (36) and (72) can be removed [31] by redefining the magnetic field contribution in the tree-level free energy as 


$$
\begin{aligned}
F_{0}= & \frac{B^{2}}{2} \rightarrow \frac{B^{2}}{2}\left[1-\frac{4 N_{c} g^{4} C_{F}^{2}}{9 \epsilon} \frac{M_{B}^{4}}{B^{2}}+\frac{m_{D}^{2} \delta m_{D}^{2}}{\epsilon(4 \pi)^{2} B^{2}}-\sum_{f} \frac{g^{2} q_{f}^{2}}{(12 \pi)^{2}} \frac{2 T^{2}}{m_{f}^{2}}\left[\frac{1}{\epsilon}\right.\right. \\
& \left.+3 \hat{m}_{D}^{2}\left\{\frac{1-\ln 2}{\epsilon^{2}}+\frac{1}{\epsilon}\left(\frac{7}{2}-\frac{\pi^{2}}{6}-\ln ^{2}(2)-2\left(\gamma_{E}+\ln \frac{\hat{\Lambda}}{2}\right)(\ln 2-1)\right)\right\}\right] \\
& +\sum_{f} \frac{g^{2} q_{f}^{2}}{(12 \pi)^{2}} \frac{\pi T}{16 m_{f}}\left[\left\{\frac{3}{8 \epsilon^{2}}+\frac{1}{\epsilon}\left(\frac{21}{8}+\frac{3}{4} \frac{\zeta^{\prime}(-1)}{\zeta(-1)}+\frac{27}{4} \ln 2+\frac{3}{4} \ln \frac{\hat{\Lambda}}{2}\right)\right.\right. \\
& +\frac{3}{4} \hat{m}_{D}^{2}\left[\frac{1}{\epsilon^{2}}\left(5 \pi^{2}-\frac{609}{10}+\frac{114 \ln 2}{5}\right)+\frac{1}{\epsilon}\left(30 \zeta(3)-\frac{5779}{75}+\frac{121}{6} \pi^{2}+\frac{114}{5} \ln ^{2}(2)\right.\right. \\
& \left.\left.\left.+\frac{468}{25} \ln 2+\left(\gamma_{E}+\ln \frac{\hat{\Lambda}}{2}\right)\left(10 \pi^{2}-\frac{609}{5}+\frac{228}{5} \ln 2\right)\right)\right]\right\}+\frac{8}{3 \pi}\left\{\frac{3 \ln 2-4}{2 \epsilon}\right. \\
& +3 \hat{m}_{D}^{2}\left[\frac{1}{40 \epsilon^{2}}\left(11+5 \pi^{2}-92 \ln 2\right)+\frac{1}{\epsilon}\left(\frac{3}{4} \zeta(3)+\frac{1557}{200}-\frac{\pi^{2}}{3}-\frac{23}{10} \ln ^{2}(2)\right.\right. \\
& \left.\left.\left.\left.-\frac{168}{25} \ln 2+\left(\gamma_{E}+\ln \frac{\hat{\Lambda}}{2}\right)\left(\frac{11}{20}+\frac{\pi^{2}}{4}-\frac{23}{5} \ln 2\right)\right)\right]\right\}\right] .
\end{aligned}
$$

So, the renormalized total free energy becomes

$$
F=F_{q}^{r}+F_{g}^{r}
$$

where

$$
\begin{aligned}
F_{q}^{r}= & N_{c} N_{f}\left[-\frac{7 \pi^{2} T^{4}}{180}\left(1+\frac{120 \hat{\mu}^{2}}{7}+\frac{240 \hat{\mu}^{4}}{7}\right)+\frac{g^{2} C_{F} T^{4}}{48}\left(1+4 \hat{\mu}^{2}\right)\left(1+12 \hat{\mu}^{2}\right)+\frac{g^{4} C_{F}^{2} T^{4}}{768 \pi^{2}}\left(1+4 \hat{\mu}^{2}\right)^{2}\left(\pi^{2}-6\right)\right. \\
& \left.+\frac{g^{4} C_{F}^{2}}{27 N_{f}} M_{B}^{4}\left(12 \ln \frac{\hat{\Lambda}}{2}-6 \aleph(z)+\frac{36 \zeta(3)}{\pi^{2}}-2-\frac{72}{\pi^{2}}\right)\right]
\end{aligned}
$$

and

$$
\begin{aligned}
\frac{F_{g}^{r}}{d_{A}}= & -\frac{\pi^{2} T^{4}}{45}\left[1-\frac{15}{2} \hat{m}_{D}^{2}+30\left(\hat{m}_{D}^{w}\right)^{3}+\frac{45}{8} \hat{m}_{D}^{4}\left(2 \ln \frac{\hat{\Lambda}}{2}-7+2 \gamma_{E}+\frac{2 \pi^{2}}{3}\right)\right] \\
& -\pi^{2} T^{4} \hat{m}_{D}^{2} \delta \hat{m}_{D}^{2}\left(\gamma_{E}+\ln \hat{\Lambda}\right)+\sum_{f} \frac{g^{2}\left(q_{f} B\right)^{2}}{(12 \pi)^{2}} \frac{T^{2}}{m_{f}^{2}}\left[4.97+2 \ln \frac{\hat{\Lambda}}{2}\right. \\
& \left.+3 \hat{m}_{D}^{2}\left\{2(1-\ln 2) \ln ^{2} \frac{\hat{\Lambda}}{2}+2\left(\frac{7}{2}-\frac{\pi^{2}}{6}-\ln ^{2}(2)-2 \gamma_{E}(\ln 2-1)\right) \ln \frac{\hat{\Lambda}}{2}+4.73\right\}\right] \\
& -\sum_{f} \frac{g^{2}\left(q_{f} B\right)^{2}}{(12 \pi)^{2}} \frac{\pi T}{32 m_{f}}\left[\left\{\frac{3}{4} \ln \frac{\hat{\Lambda}}{2}+2 \ln \frac{\hat{\Lambda}}{2}\left(\frac{21}{8}+\frac{3}{4} \frac{\zeta^{\prime}(-1)}{\zeta(-1)}+\frac{27}{4} \ln 2\right)+43.566\right.\right. \\
& +\frac{3}{4} \hat{m}_{D}^{2}\left[2 \ln 2 \frac{\hat{\Lambda}}{2}\left(5 \pi^{2}-\frac{609}{10}+\frac{114 \ln 2}{5}\right)+2 \ln \frac{\hat{\Lambda}}{2}\left(30 \zeta(3)-\frac{5779}{75}+\frac{121}{6} \pi^{2}+\frac{114}{5} \ln ^{2}(2)\right.\right. \\
& \left.\left.\left.+\frac{468}{25} \ln 2+\gamma_{E}\left(10 \pi^{2}-\frac{609}{5}+\frac{228}{5} \ln 2\right)\right)+106.477\right]\right\}+\frac{8}{3 \pi}\left\{(3 \ln 2-4) \ln \frac{\hat{\Lambda}}{2}-3.92\right. \\
& +3 \hat{m}_{D}^{2}\left[\frac{1}{20} \ln ^{2} \frac{\hat{\Lambda}}{2}\left(11+5 \pi^{2}-92 \ln 2\right)+2 \ln \frac{\hat{\Lambda}}{2}\left(\frac{3}{4} \zeta(3)+\frac{1557}{200}-\frac{\pi^{2}}{3}-\frac{23}{10} \ln ^{2}(2)\right.\right. \\
& \left.\left.\left.-\frac{168}{25} \ln 2+\gamma_{E}\left(\frac{11}{20}+\frac{\pi^{2}}{4}-\frac{23}{5} \ln 2\right)\right)-1.86\right]\right\} .
\end{aligned}
$$



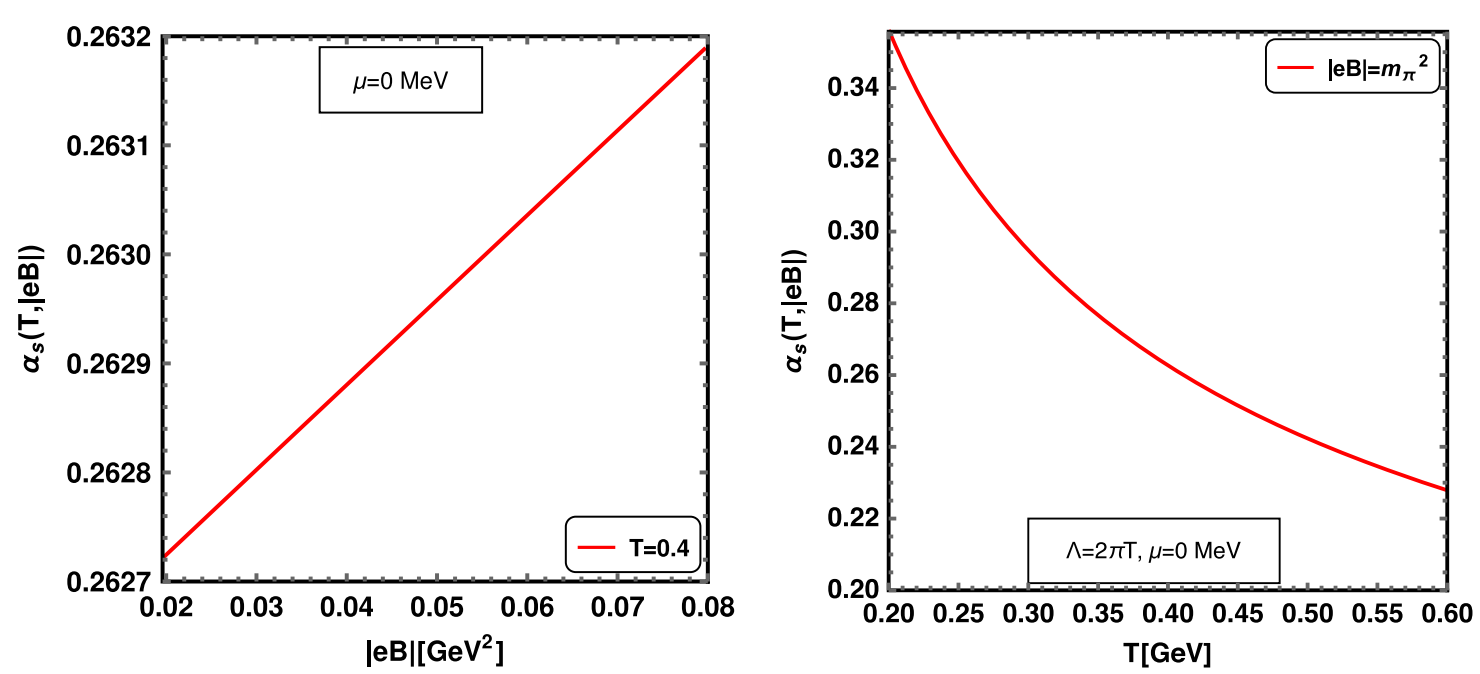

FIG. 4. Left: variation of the one-loop QCD coupling with weak magnetic field, $|e B|$ for $T=0.4 \mathrm{GeV}$. Right: variation with temperature, $T$ for $|e B|=m_{\pi}^{2}$.

\section{B. Pressure}

The expression for the pressure of hot and dense QCD matter in one-loop HTLpt in the presence of a weak magnetic field can now be written directly from the oneloop free energy as

$$
P(T, \mu, B, \Lambda)=-F(T, \mu, B, \Lambda),
$$

whereas the ideal gas limit of the pressure reads as

$$
\begin{aligned}
P_{\text {Ideal }}(T, \mu)= & \frac{B^{2}}{2}+N_{c} N_{f} \frac{7 \pi^{2} T^{4}}{180}\left(1+\frac{120}{7} \hat{\mu}^{2}+\frac{240}{7} \hat{\mu}^{4}\right) \\
& +\left(N_{c}^{2}-1\right) \frac{\pi^{2} T^{4}}{45} .
\end{aligned}
$$

\section{STRONG COUPLING AND SCALES}

The one-loop running coupling which evolves with both the momentum transfer and the magnetic field is recently obtained in Ref. [105] as

$$
\alpha_{s}\left(\Lambda^{2},|e B|\right)=\frac{\alpha_{s}\left(\Lambda^{2}\right)}{1+b_{1} \alpha_{s}\left(\Lambda^{2}\right) \ln \left(\frac{\Lambda^{2}}{\Lambda^{2}+|e B|}\right)},
$$

in the domain $|e B|<\Lambda^{2}$ where the one-loop running coupling at renormalization scale reads as

$$
\alpha_{s}\left(\Lambda^{2}\right)=\frac{1}{b_{1} \ln \left(\Lambda^{2} / \Lambda_{\overline{\mathrm{MS}}}^{2}\right)},
$$

with $\quad b_{1}=\frac{11 N_{c}-2 N_{f}}{12 \pi}, \quad \Lambda_{\overline{\mathrm{MS}}}=176 \mathrm{MeV} \quad[106] \quad$ at $\alpha_{s}(1.5 \mathrm{GeV})=0.326$ for $N_{f}=3$. We note here that we choose separate renormalization scales for gluon $\Lambda=\Lambda_{g}$, for quark $\Lambda=\Lambda_{q}$, which are chosen at their central values, respectively, as $\Lambda_{g}=2 \pi T$ and $\Lambda_{q}=2 \pi \sqrt{T^{2}+\mu^{2} / \pi^{2}}$. The renormalization scales can be varied by a factor of 2 with respect to its central value. On the other hand the magnetic field strength can also be varied as long as $|e B|>\Lambda^{2}$ for strong field and $|e B|<\Lambda^{2}$ for weak field approximation for a given temperature vis-à-vis the renormalization scale, as discussed in Sec. III. The left panel of Fig. 4 displays running of $\alpha_{s}$ with $|e B|$ as the central value of the renormalization scale $\Lambda_{g}=\Lambda_{q}=2 \pi T \mathrm{GeV}$ for $T=0.4 \mathrm{GeV}$. This indicates a slow increase of $\alpha_{s}$ with the increase of $|e B|$ in the domain $|e B|<\Lambda^{2}$. On the other hand the right panel of Fig. 4 exhibits running of $\alpha_{s}$ with $T$ for $|e B|=m_{\pi}^{2}$. This behavior also consolidates that $\alpha_{s}$ runs very slowly with $|e B|$.

\section{COMPARISON WITH FULL RESULTS}

In this section we try to justify the use of high $T$ expansion in our calculation by comparing it with the full results (without high $T$ expansion) in the spirit of HTL perturbation theory. The full result is computed numerically and the detailed calculations are given in Appendix D. Figure 5 displays a comparison between the scaled pressure of QCD matter computed with and without high temperature expansion for various field strengths. The solid lines represent results from high $T$ expansion whereas dashed lines correspond to the results of without high $T$ expansion. As can be seen from the figure, the two plots (i.e., with and without high $T$ expansion) for a given magnetic field strength do not differ much except at low $T$. Thus we can use the high $T$ expansion to get the analytical expression of the pressure. Although this high $T$ expansion is not in the spirit of HTL perturbation theory, it nevertheless is very effective (especially in higher order loops), because in this 


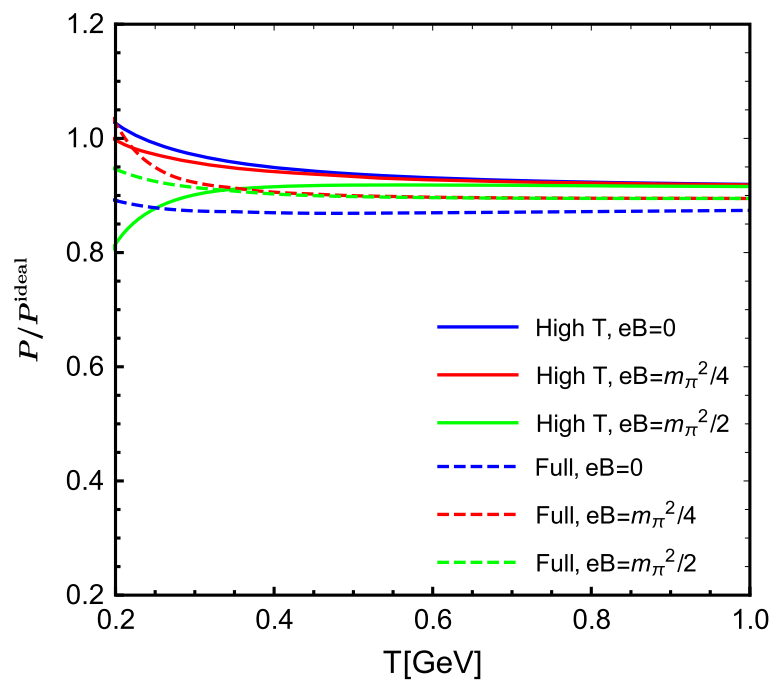

FIG. 5. Comparison of pressure with and without high $T$ expansion for $e B=0, m_{\pi}^{2} / 4$ and $m_{\pi}^{2} / 2$ with $N_{f}=2$. "Full" represents without high $T$ expansion.

way one can bypass the tiresome work of a quasiparticle pole as well as Landau damping calculations. For simplicity, this high $T$ expansion in the absence of a magnetic field has widely been used in the literature for leading order, next-to-leading order, and next-to-next-to-leading order in HTL. In the same spirit we have used such high $T$ expansion here in the presence of a magnetic field and hereafter we show the results with high $T$ expansion.

\section{RESULTS}

In this section we discuss the main results of this paper. In Fig. 6 we display the temperature variation of scaled

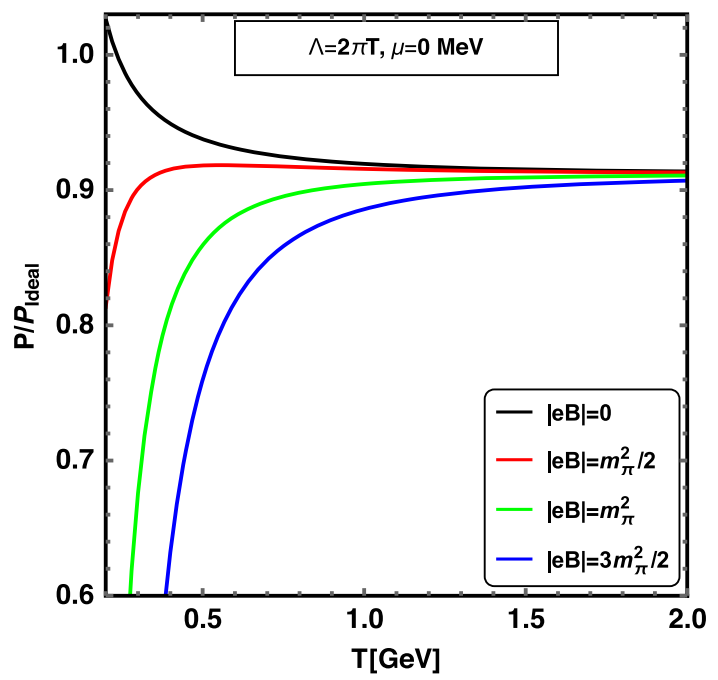

pressure with that of ideal gas value for hot and dense magnetized QCD matter in one-loop HTLpt within a weak field approximation for different values of field strengths, $|e B|=0, m_{\pi}^{2} / 2, m_{\pi}^{2}$, and $3 m_{\pi}^{2} / 2$. The left panel shows vanishing chemical potential $\mu=0$ whereas the right panel shows $\mu=0.3 \mathrm{GeV}$. We note that for $|e B|=0$ one gets back usual one-loop HTLpt pressure [69-77]. From both plots one can observe that the low $T(<0.8 \mathrm{GeV})$ behavior of the pressure is strongly affected by the presence of a magnetic field whereas at high $T(\geq 0.8 \mathrm{GeV})$ it almost remains unaffected as the temperature becomes the dominant scale because of weak field approximation $|e B|<$ $m_{\mathrm{th}}^{2}<T^{2}$. Nevertheless, we also note a specific difficulty one encounters with HTLpt $(|e B|=0)$. This has to do with the fact that the one-loop HTLpt introduces an overcounting of some contributions [69-76] in a strong coupling $(g)$. This is because the loop expansion and the coupling expansion are not symmetrical in HTLpt. So, at each loop order in HTLpt the result is an infinite series in $g$. At leading order in HTLpt one only gets the correct perturbative coefficients for $g^{0}$ and $g^{3}$ when one expands in power of $g$. Thus, for a given order in $g$ higher loop orders contribute and this is only corrected by extending the calculation up to higher loop orders [78-88]. We also note that the pressure is slightly reduced in the presence of a $\mu$ (right panel) than that of $\mu=0$, for a given $|e B|$.

It is seen from Fig. 7 that the slope of the curve decreases with the increase of $T$. So, Fig. 7 also consolidates the fact that in weak field approximation the effect of the magnetic field diminishes with an increase of $T$. This indicates that the magnetic field is the dominant scale at low $T$ and becomes negligible at high $T$.

To check the sensitivity with the renormalization scale $\Lambda_{q, g}$ in Fig. 8 we have displayed the temperature variation

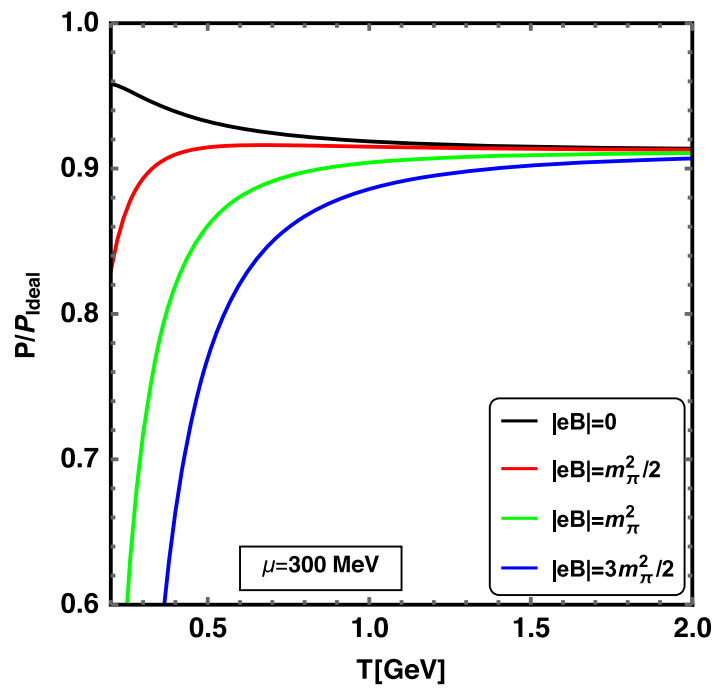

FIG. 6. Variation of the scaled one-loop pressure with temperature for $N_{f}=2$ with $\mu=0$ (left) and for $\mu=300 \mathrm{MeV}$ (right) in the presence of a weak magnetic fields of various strengths, $|e B|=0, m_{\pi}^{2} / 2, m_{\pi}^{2}$, and $3 m_{\pi}^{2} / 2$. In the right panel for $\mu \neq 0$, the renormalization scales are defined in the text in Sec. VII. 

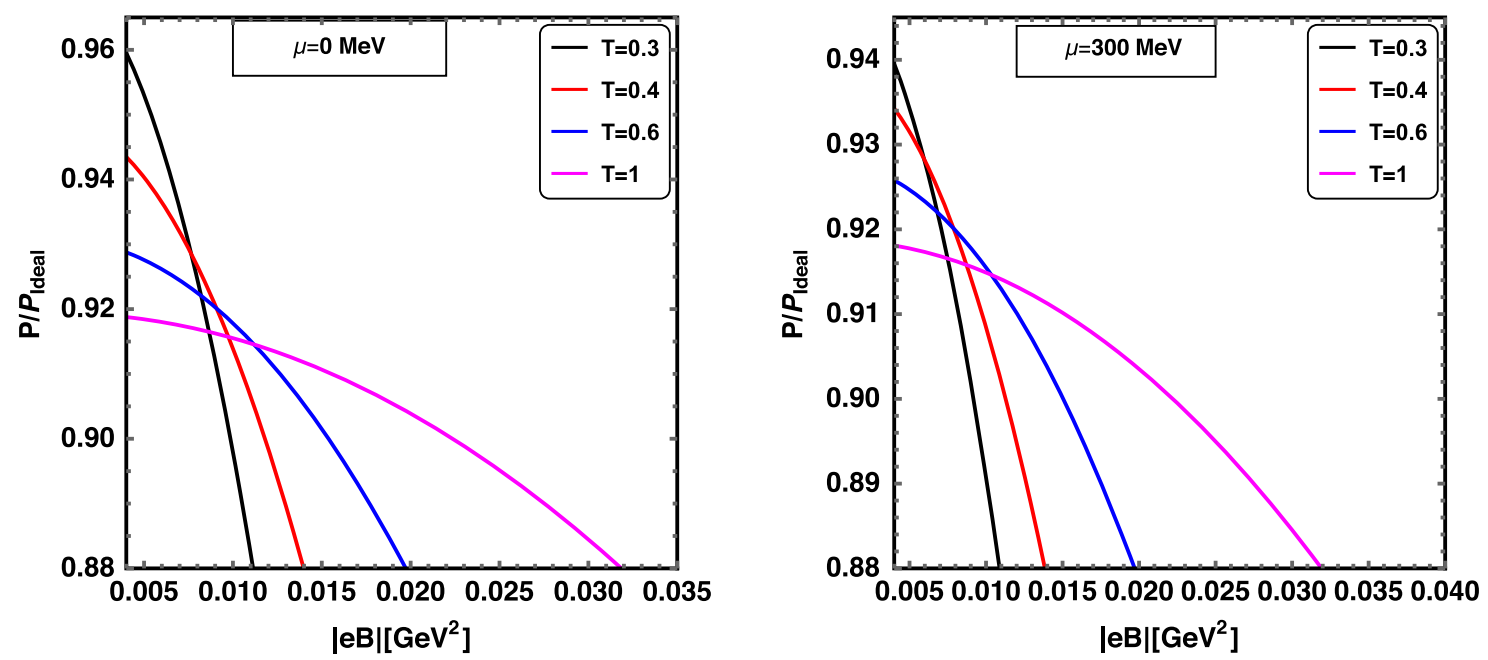

FIG. 7. Variation of the scaled one-loop pressure with magnetic field for $N_{f}=2$ with $\mu=0$ (left) and $\mu=300 \mathrm{MeV}$ (right) for $T=(0.3,0.4,0.6$ and 1$) \mathrm{GeV}$. The renormalization scales are defined in the text in Sec. VII.
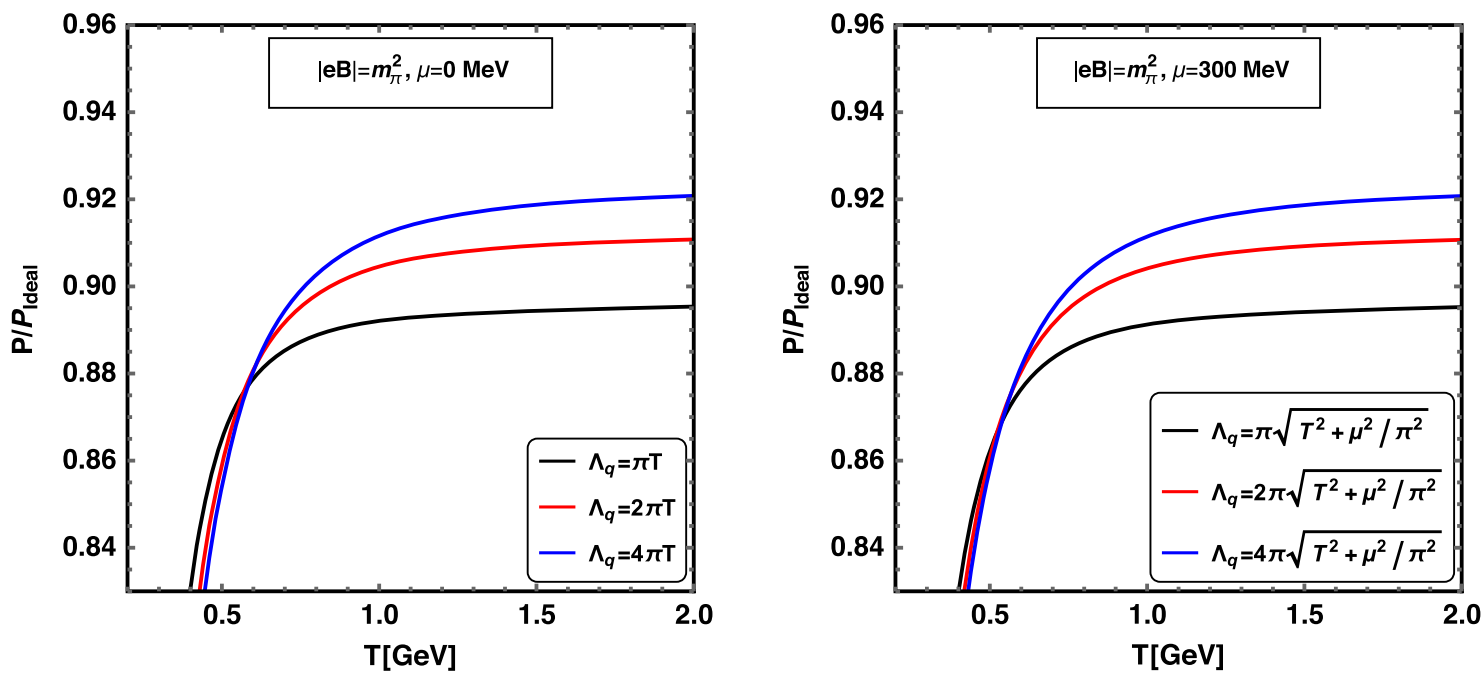

FIG. 8. Variation of the scaled one-loop pressure with temperature for $N_{f}=2$ with $\mu=0$ (left) and $\mu=300 \mathrm{MeV}$ (right) in the presence of a weak magnetic field of strength $e B=m_{\pi}^{2}$ for different values of a renormalization scale of gluons. $\Lambda_{g}=\pi T, 2 \pi T$, and $4 \pi T$ and scale of quarks is given in the inset.

of the scaled one-loop pressure in the presence of a constant weak magnetic field by varying $\Lambda_{q, g}$ by a factor of 2 around its central value for both zero and finite chemical potential. It is found to depend moderately on the renormalization scale $\Lambda_{q, g}$. One may need higher loops calculation and log resummation to further reduce the renormalization scale dependent band.

\section{CONCLUSION}

In this paper we presented a systematic framework based on the general structure of two-point functions of a fermion and a gauge boson to evaluate the QCD pressure in nontrivial backgrounds, viz., when both heat bath and magnetic field are considered together. This framework has been applied to the case when the heat bath is weakly magnetized. The total pressure of a magnetized hot and dense deconfined QCD matter is the sum of three contributions coming from (a) the quark part, (b) the gluonic part, and (c) the tree-level free energy due to the constant magnetic field. We note that the presence of an external magnetic field affects both the fermion and gluon effective two-point (self-energy and propagator) functions. We have also used strong coupling that runs through both renormalization scale and magnetic field strength. Although gluons are electrically charge neutral, they are mostly affected through the quark loop because quark propagators get modified in the presence of a background magnetic 
field. Based on the most general structure of the effective two-point functions, the quark propagator in our earlier calculation [47] and gluon propagator in paper-I [45], we obtain the QCD Debye screening mass, gluon and quark free energy in one-loop HTLpt in the presence of a weak field approximation. The divergences appeared therein are taken care of by redefining the magnetic field in the treelevel free energy term and through an HTL counterterm. Using high temperature expansion we found finite results which are also completely analytic and gauge independent but depend on renomalization scale and magnetic field strength. We have again compared the results with the numerically evaluated full results thereby justifying the use of the high temperature expansion. We have also discussed in details the modification of QCD Debye mass which depends on three scales, viz., the thermal quark mass, temperature, and the magnetic field. The weak field pressure is strongly affected at low $T(<0.8 \mathrm{GeV})$ beyond which the HTL result takes over. We have checked the sensitivity of the pressure on the various scales, viz., the renormalization and magnetic field strength. The result is sensitive to the renormalization scale as it produces band while varying its value by a factor of 2 . The sensitivity of pressure on the magnetic field is strong at low $T$ and negligible at high $T$. We have also outlined a general drawback with one-loop HTLpt that introduces an overcounting of some contributions, as a remedy of which one needs to push the calculation to higher loop orders.

\section{ACKNOWLEDGMENTS}

The authors gratefully acknowledge a valuable discussion with J. Kapusta on a technical issue in the calculation. The authors would also like to acknowledge useful discussions with Arghya Mukherjee, Jens O. Andersen, and Michael Strickland. A. B., B. K., and M. G. M. were funded by the Department of Atomic Energy, India, via the project TPAES. A. B. was also partially supported by the National Post Doctoral Program CAPES, Government of Brazil. N. H. gratefully acknowledges financial support from the Alexander von Humboldt Foundation, Germany, and also from the Department of Atomic Energy, India.

\section{APPENDIX A: FERMIONIC SUM INTEGRALS}

The dimensionally regularized sum integrals are defined as

$$
\sum_{\{P\}}=\left(\frac{e^{\gamma_{E}} \Lambda^{2}}{4 \pi}\right)^{\epsilon} T \sum_{\substack{p_{0}=i \omega_{n} \\ \omega_{n}=(2 n+1) \pi T-i \mu}} \int \frac{d^{d-2 \epsilon} p}{(2 \pi)^{d-2 \epsilon}},
$$

where $\Lambda$ can be identified as the $\overline{\mathrm{MS}}$ renormalization scale which also introduces the factor $\left(\frac{e^{\top} E}{4 \pi}\right)^{\epsilon}$ along with it, with $\gamma_{E}$ being the Euler-Mascheroni constant. Before listing all of the sum integrals used in our paper, we note that they are interrelated among themselves via

$$
\sum_{\{P\}} \frac{1}{P^{4}}=-\frac{d-2}{2} \sum_{\{P\}} \frac{1}{p^{2} P^{2}}=\frac{d-5}{d-4} \bigvee_{\{P\}} \frac{\mathcal{T}_{P}}{P^{4}} .
$$

\section{Simple one-loop sum integrals}

The list of fermionic sum integrals needed are

$$
\begin{gathered}
y_{\{P\}} \frac{1}{P^{2}}=\frac{T^{2}}{24}\left(\frac{\Lambda}{4 \pi T}\right)^{2 \epsilon}\left[1+12 \hat{\mu}^{2}\right. \\
\left.+2 \epsilon\left(1+12 \hat{\mu}^{2}+12 \aleph(1, z)\right)\right], \\
\sum_{\{P\}} \frac{1}{P^{4}}=\frac{1}{(4 \pi)^{2}}\left(\frac{\Lambda}{4 \pi T}\right)^{2 \epsilon}\left[\frac{1}{\epsilon}-\aleph(z)\right], \\
\sum_{\{P\}} \frac{p^{2}}{P^{6}}=-\frac{3}{4} \frac{1}{(4 \pi)^{2}}\left(\frac{\Lambda}{4 \pi T}\right)^{2 \epsilon}\left[\frac{1}{\epsilon}-\frac{2}{3}-\aleph(z)\right], \\
y_{\{P\}} \frac{1}{p^{2} P^{2}}=-\frac{2}{(4 \pi)^{2}}\left(\frac{\Lambda}{4 \pi T}\right)^{2 \epsilon}\left[\frac{1}{\epsilon}+2-\aleph(z)\right], \\
\sum_{\{P\}} \frac{p_{3}^{2}}{p^{2} P^{4}}=\frac{1}{3(4 \pi)^{2}}\left(\frac{\Lambda}{4 \pi T}\right)^{2 \epsilon}\left[\frac{1}{\epsilon}+\frac{2}{3}-\aleph(z)\right], \\
\sum_{\{P\}} \frac{p_{3}^{2}}{p^{4} P^{2}}=-\frac{2}{3(4 \pi)^{2}}\left(\frac{\Lambda}{4 \pi T}\right)^{2 \epsilon}\left[\frac{1}{\epsilon}+\frac{8}{3}-\aleph(z)\right] .
\end{gathered}
$$

For some frequently occurring combinations of special functions we applied the following abbreviations:

$$
\begin{gathered}
\zeta^{\prime}(x, y) \equiv \partial_{x} \zeta(x, y), \\
\aleph(n, z) \equiv \zeta^{\prime}(-n, z)+(-1)^{n+1} \zeta^{\prime}\left(-n, z^{*}\right), \\
\aleph(z) \equiv \Psi(z)+\Psi\left(z^{*}\right),
\end{gathered}
$$

where $n$, in Eq. (A10), is assumed to be an integer and $z$ a general complex number, here $z=1 / 2-i \hat{\mu}$. Here $\zeta$ and $\Psi$ denote the Riemann zeta function and the digamma function respectively and $\Psi$ can be expressed as

$$
\Psi(z) \equiv \frac{\Gamma^{\prime}(z)}{\Gamma(z)} .
$$

Below we enlist the values of the function $\aleph$ as required for our calculation. Although the following list $\mathrm{s}$ are given at small values of $\mu / T$, in the actual plot we calculate $\aleph$ for any value of $\mu$ using Mathematica. 


$$
\begin{aligned}
\aleph(z)= & -2 \gamma_{E}-4 \ln 2+14 \zeta(3) \hat{\mu}^{2}-62 \zeta(5) \hat{\mu}^{4} \\
+ & 254 \zeta(7) \hat{\mu}^{6}+\mathcal{O}\left(\hat{\mu}^{8}\right), \\
\aleph(1, z)= & -\frac{1}{12}\left(\ln 2-\frac{\zeta^{\prime}(-1)}{\zeta(-1)}\right)-\left(1-2 \ln 2-\gamma_{E}\right) \hat{\mu}^{2} \\
& -\frac{7}{6} \zeta(3) \hat{\mu}^{4}+\frac{31}{15} \zeta(5) \hat{\mu}^{6}+\mathcal{O}\left(\hat{\mu}^{8}\right) .
\end{aligned}
$$

$$
\bigcup_{\{P\}} \frac{p_{3}^{2}}{p^{4} P^{2}} \mathcal{T}_{P}^{2}=\Delta_{4}^{\prime \prime} \Delta_{0}^{\prime} \bigcup_{\{P\}} \frac{1}{p^{2} P^{2}}=-\frac{2}{d-2} \Delta_{4}^{\prime \prime} \Delta_{0}^{\prime} \bigvee_{\{P\}} \frac{1}{P^{4}},
$$

$$
\bigcup_{\{P\}} \frac{p_{3}^{2}}{p^{4} P^{2}} \mathcal{T}_{P}=\Delta_{3} \Delta_{0}^{\prime} \bigcup_{\{P\}} \frac{1}{p^{2} P^{2}}=-\frac{2}{d-2} \Delta_{3} \Delta_{0}^{\prime} \bigvee_{\{P\}} \frac{1}{P^{4}},
$$

\section{HTL one-loop sum integrals for weak field case}

We also need some more difficult one-loop sum integrals that involve the angular average defined earlier in Eq. (24). For brevity, henceforth we will use the notation $c=\cos \theta$ for single angular average and $c_{i}=\cos \theta_{i}$ for multiple angular averages. We list the sum integrals below. The expressions for the respective angular averages appearing in the process and denoted by $\Delta_{i}$ 's are given in Appendix C.

$$
\begin{aligned}
& \bigcup_{\{P\}} \frac{p_{3}^{2}}{p^{2} P^{4}} \mathcal{T}_{P}^{2}=\Delta_{0}{ }^{\prime \prime} \bigcup_{\{P\}}\left(\frac{p_{3}^{2}}{p^{2} P^{4}}+\frac{p_{3}^{2}}{p^{4} P^{2}}\right)+\Delta_{10} \bigcup_{\{P\}} \frac{p_{3}^{2}}{p^{4} P^{2}} \\
& =\left(\frac{d-4}{d-2} \Delta_{0}^{\prime \prime} \Delta_{0}^{\prime}-\frac{2}{d-2} \Delta_{10} \Delta_{0}^{\prime}\right) \sum_{\{P\}} \frac{1}{P^{4}},
\end{aligned}
$$

$$
\sum_{\{P\}} \frac{1}{P^{4}} \mathcal{T}_{P}=\frac{d-4}{d-5} \bigvee_{\{P\}} \frac{1}{P^{4}}
$$

$$
\begin{aligned}
& \underbrace{}_{\{P\}} \frac{p_{3}^{2}}{p^{2} P^{4}} \mathcal{T}_{P}=\left(1+\Delta_{0}\right) \Delta_{0}^{\prime}\left(\bigcup_{\{P\}} \frac{1}{P^{4}}+\bigotimes_{\{P\}} \frac{1}{p^{2} P^{2}}\right) \\
&-\Delta_{3}^{\prime} \Delta_{0}^{\prime} \bigcup_{\{P\}} \frac{1}{p^{2} P^{2}} \\
&=\left(\frac{d-4}{d-2}\left(1+\Delta_{0}\right) \Delta_{0}^{\prime}+\frac{2}{d-2} \Delta_{3}^{\prime} \Delta_{0}^{\prime}\right) \bigotimes_{\{P\}} \frac{1}{P^{4}} .
\end{aligned}
$$

$$
\begin{aligned}
& \bigcup_{\{P\}} \frac{1}{P^{4}} \mathcal{T}_{P}^{2}=\left(\frac{d-4}{d-2} \Delta_{0}^{\prime \prime}-\frac{2}{d-2} \Delta_{10}\right) \bigcup_{\{P\}} \frac{1}{P^{4}}, \\
& \bigcup_{\{P\}} \frac{p_{3}^{2}}{p_{0}^{2} P^{4}} \mathcal{T}_{P}^{2}=\left(\frac{\Delta_{0}^{\prime \prime}}{d}-\frac{2}{d(d-2)} \Delta_{11}\right) \bigcup_{\{P\}} \frac{1}{P^{4}},
\end{aligned}
$$

\section{APPENDIX B: BOSONIC SUM INTEGRALS}

\section{Simple one-loop sum integrals}

To evaluate the sum integrals over the external bosonic momenta, we use the following master formula:

$$
\bigcup_{P} \frac{p_{\perp}^{i} p_{3}^{j}}{p^{m} P^{n}}=\left\langle c^{j}\left(1-c^{2}\right)^{\frac{i}{2}}\right\rangle_{c} \bigvee_{P} \frac{1}{p^{m-i-j} P^{n}},
$$

thus eventually requiring the following basis integrals:

$$
\begin{aligned}
\bigvee_{P} \frac{1}{P^{2}}= & -\frac{T^{2}}{12}\left(\frac{\Lambda}{4 \pi T}\right)^{2 \epsilon}\left[1+2 \epsilon\left(1+\frac{\zeta^{\prime}(-1)}{\zeta(-1)}\right)+\epsilon^{2}\left(4 \gamma_{E}\left(1+\frac{\zeta^{\prime}(-1)}{\zeta(-1)}-\frac{\gamma_{E}}{2}-\ln 2 \pi\right)\right.\right. \\
& \left.\left.+4 \ln 2 \pi\left(1+\frac{\zeta^{\prime}(-1)}{\zeta(-1)}\right)+\frac{12 \zeta^{\prime \prime}(2)}{\pi^{2}}+\frac{\pi^{2}}{12}-2 \ln ^{2}(2 \pi)\right)\right]+\mathcal{O}[\epsilon]^{3}
\end{aligned}
$$




$$
\begin{aligned}
& \bigvee_{P} \frac{1}{p^{2} P^{2}}=-\frac{2}{(4 \pi)^{2}}\left(\frac{\Lambda}{4 \pi T}\right)^{2 \epsilon}\left[\frac{1}{\epsilon}+2 \gamma_{E}+2+\epsilon\left(4+4 \gamma_{E}+\frac{\pi^{2}}{4}-4 \gamma_{1}\right)\right. \\
&\left.+\frac{\epsilon^{2}}{6}\left(3\left(8\left(-2 \gamma_{1}+\gamma_{2}+2\right)+\pi^{2}+\gamma_{E}\left(16+\pi^{2}\right)\right)-14 \zeta(3)\right)\right]+\mathcal{O}[\epsilon]^{3}, \\
& \bigcup_{P} \frac{1}{P^{4}}=\frac{1}{(4 \pi)^{2}}\left(\frac{\Lambda}{4 \pi T}\right)^{2 \epsilon}\left[\frac{1}{\epsilon}+2 \gamma_{E}+\epsilon\left(\frac{\pi^{2}}{4}-4 \gamma_{1}\right)+\frac{\epsilon^{2}}{6}\left(24 \gamma_{2}-14 \zeta(3)+3 \gamma_{E} \pi^{2}\right)\right]+\mathcal{O}[\epsilon]^{3} .
\end{aligned}
$$

\section{HTL one-loop sum integrals}

Similarly as in the fermionic part, here also we list the one-loop bosonic HTL integrals required for our computation. We define the following master HTL integrals which is largely required to compute the HTL sum integrals appearing in the expression for free energies.

1.

$$
\begin{aligned}
\bigvee_{P} \frac{p_{\perp}^{i} p_{3}^{j} p_{0}^{k}}{p^{m}} \mathcal{T}_{P} & =\left\langle c^{m-i-j-d}\right\rangle_{c} \bigvee_{P} \frac{p_{\perp}^{i} p_{3}^{j} p_{0}^{k+2}}{p^{m} P^{2}} \\
& =\left\langle c^{m-i-j-d}\right\rangle_{c} \bigvee_{P} \frac{p_{\perp}^{i} p_{3}^{j}}{p^{m-k-2} P^{2}} \\
& =\left\langle c_{1}^{m-i-j-d}\right\rangle_{c_{1}}\left\langle c_{2}^{j}\left(1-c^{2}\right)^{\frac{i}{2}}\right\rangle_{c_{2}} \bigcup_{P} \frac{1}{p^{m-k-i-j-2} P^{2}}
\end{aligned}
$$

2.

$$
\begin{aligned}
\bigcup_{P} \frac{p_{\perp}^{i} p_{3}^{j} p_{0}^{k}}{p^{m} P^{2}} \mathcal{T}_{P} & =\left\langle\frac{1-c^{m+2-i-j-d}}{1-c^{2}}\right\rangle_{c} \sum_{P} \frac{p_{\perp}^{i} p_{3}^{j} p_{0}^{k+2}}{p^{m+2} P^{2}} \\
& =\left\langle\frac{1-c^{m+2-i-j-d}}{1-c^{2}}\right\rangle \sum_{c} \sum_{P} \frac{p_{\perp}^{i} p_{3}^{j}}{p^{m-k} P^{2}} \\
& =\left\langle\frac{1-c_{1}^{m+2-i-j-d}}{1-c_{1}^{2}}\right\rangle_{c_{1}}\left\langle c_{2}^{j}\left(1-c_{2}^{2}\right)^{\frac{i}{2}}\right\rangle_{c_{2}} \bigvee_{P} \frac{1}{p^{m-k-i-j} P^{2}}
\end{aligned}
$$

3.

$$
\begin{aligned}
& \bigvee_{P} \frac{p_{\perp}^{i} p_{3}^{j} p_{0}^{k}}{p^{m} P^{4}} \mathcal{T}_{P}=\left\langle\frac{1}{1-c^{2}}\right\rangle{ }_{c} \bigcup_{P} \frac{p_{\perp}^{i} p_{3}^{j} p_{0}^{k+2}}{p^{m+2} P^{4}}-\left\langle\frac{1-c^{m+4-i-j-d}}{\left(1-c^{2}\right)^{2}}\right\rangle_{c} \bigcup_{P} \frac{p_{\perp}^{i} p_{3}^{j} p_{0}^{k+2}}{p^{m+4} P^{2}} \\
& =\left\langle\frac{1}{1-c^{2}}\right\rangle_{c} \bigvee_{P} \frac{p_{\perp}^{i} p_{3}^{j}}{p^{m-k} P^{4}}+\left\langle\frac{1}{1-c^{2}}\right\rangle \sum_{c} Y_{P}\left(\frac{k}{2}+1\right) \frac{p_{\perp}^{i} p_{3}^{j}}{p^{m+2-k} P^{2}}-\left\langle\frac{1-c^{m+4-i-j-d}}{\left(1-c^{2}\right)^{2}}\right\rangle \sum_{c} \bigvee_{P} \frac{p_{\perp}^{i} p_{3}^{j}}{p^{m-k+2} P^{2}} \\
& =\left\langle\frac{1}{1-c_{1}^{2}}\right\rangle_{c_{1}}\left\langle c_{2}^{j}\left(1-c_{2}^{2}\right)^{\frac{i}{2}}\right\rangle_{c_{2}} \bigvee_{P} \frac{1}{p^{m-k-i-j} P^{4}}+\left\langle\frac{1}{1-c_{1}^{2}}\right\rangle_{c_{1}}\left\langle c_{2}^{j}\left(1-c_{2}^{2}\right)^{\frac{i}{2}}\right\rangle_{c_{2}} \bigvee_{P}\left(\frac{k}{2}+1\right) \frac{1}{p^{m+2-k-i-j} P^{2}} \\
& -\left\langle\frac{1-c_{1}^{m+4-i-j-d}}{\left(1-c_{1}^{2}\right)^{2}}\right\rangle_{c_{1}}\left\langle c_{2}^{j}\left(1-c_{2}^{2}\right)^{\frac{i}{2}}\right\rangle_{c_{2}} \bigvee_{P} \frac{1}{p^{m-k-i-j+2} P^{2}}
\end{aligned}
$$


4.

$$
\begin{aligned}
\bigcup_{P} \frac{p_{\perp}^{i} p_{3}^{j} p_{0}^{k}}{p^{m}} \mathcal{T}_{P}^{2} & =\left\langle\frac{c_{1}^{m+2-i-j-d}-c_{2}^{m+2-i-j-d}}{c_{1}^{2}-c_{2}^{2}}\right\rangle_{c_{1}, c_{2}} \sum_{P} \frac{p_{\perp}^{i} p_{3}^{j} p_{0}^{k+4}}{p^{m+2} P^{2}} \\
& =\left\langle\frac{c_{1}^{m+2-i-j-d}-c_{2}^{m+2-i-j-d}}{c_{1}^{2}-c_{2}^{2}}\right\rangle_{c_{1}, c_{2}} \sum_{P} \frac{p_{\perp}^{i} p_{3}^{j}}{p^{m-k-2} P^{2}} \\
& =\left\langle\frac{c_{1}^{m+2-i-j-d}-c_{2}^{m+2-i-j-d}}{c_{1}^{2}-c_{2}^{2}}\right\rangle_{c_{1}, c_{2}}\left\langle c_{3}^{j}\left(1-c_{3}^{2}\right)^{\frac{i}{2}}\right\rangle_{c_{3}} \bigcup_{P} \frac{1}{p^{m-k-i-j-2} P^{2}}
\end{aligned}
$$

5.

$$
\begin{aligned}
\sum_{P} \frac{p_{\perp}^{i} p_{3}^{j} p_{0}^{k}}{p^{m} P^{2}} \mathcal{T}_{P}^{2} & =\left\langle\frac{1-c_{1}^{m+4-i-j-d}}{\left(1-c_{1}^{2}\right)\left(c_{1}^{2}-c_{2}^{2}\right)}+c_{1} \leftrightarrow c_{2}\right\rangle_{c_{1}, c_{2}} \oint_{P} \frac{p_{\perp}^{i} p_{3}^{j} p_{0}^{k+4}}{p^{m+4} P^{2}} \\
& =\left\langle\frac{1-c_{1}^{m+4-i-j-d}}{\left(1-c_{1}^{2}\right)\left(c_{1}^{2}-c_{2}^{2}\right)}+c_{1} \leftrightarrow c_{2}\right\rangle_{c_{1}, c_{2}} \sum_{P} \frac{p_{\perp}^{i} p_{3}^{j}}{p^{m-k} P^{2}} \\
& =\left\langle\frac{1-c_{1}^{m+4-i-j-d}}{\left(1-c_{1}^{2}\right)\left(c_{1}^{2}-c_{2}^{2}\right)}+c_{1} \leftrightarrow c_{2}\right\rangle_{c_{1}, c_{2}}\left\langle c_{3}^{j}\left(1-c_{3}^{2}\right)^{\frac{i}{2}}\right\rangle_{c_{3}} \sum_{P} \frac{1}{p^{m-k-i-j} P^{2}}
\end{aligned}
$$

1.

\section{a. HTL sum integrals required for longitudinal part}

$$
\begin{aligned}
& \bigvee_{P} \frac{A_{2}}{p^{2}}=\bigvee_{P} \frac{1}{2}\left[\mathcal{T}_{P}\left(\frac{1}{p^{2}}-\frac{p_{3}^{2}}{p^{4}}\right)+\left(1-\mathcal{T}_{P}\right)\left(\frac{p_{0}^{2}}{p^{4}}-\frac{3 p_{0}^{2} p_{3}^{2}}{p^{6}}\right)\right] \\
& =\left[\frac{1}{2}\left\langle c_{1}^{2-d}-c_{1}^{4-d}\right\rangle_{c_{1}}\left\langle 1-c_{2}^{2}\right\rangle_{c_{2}}+\left\langle c_{1}^{4-d}\right\rangle_{c_{1}}\left\langle c_{2}^{2}\right\rangle_{c_{2}}\right]{\underset{P}{P}}_{P^{2}} \frac{1}{P^{2}} \\
& =-\left(\frac{\Lambda}{4 \pi T}\right)^{2 \epsilon} \frac{T^{2}}{72}\left[\frac{1}{\epsilon}-\frac{1}{3}+2 \frac{\zeta^{\prime}(-1)}{\zeta(-1)}+2 \ln 2\right] \text {. }
\end{aligned}
$$

2.

$$
\begin{aligned}
y_{P} \frac{A_{2}}{p^{4}} & =\bigvee_{P} \frac{1}{2}\left[\mathcal{T}_{P}\left(\frac{1}{p^{4}}-\frac{p_{3}^{2}}{p^{6}}\right)+\left(1-\mathcal{T}_{P}\right)\left(\frac{p_{0}^{2}}{p^{6}}-\frac{3 p_{0}^{2} p_{3}^{2}}{p^{8}}\right)\right] \\
& =\left[\frac{1}{2}\left\langle c_{1}^{4-d}-c_{1}^{6-d}\right\rangle_{c_{1}}\left\langle 1-c_{2}^{2}\right\rangle_{c_{2}}+\left\langle c_{1}^{6-d}\right\rangle_{c_{1}}\left\langle c_{2}^{2}\right\rangle_{c_{2}}\right] \bigvee_{P} \frac{1}{p^{2} P^{2}} \\
& =-\left(\frac{\Lambda e^{\gamma_{E}}}{4 \pi T}\right)^{2 \epsilon} \frac{1}{3(4 \pi)^{2}}\left[\frac{1}{\epsilon}+\frac{1}{6}(1+12 \ln 2)\right]
\end{aligned}
$$

3.

$$
\begin{aligned}
\bigcup_{P} \frac{\mathcal{T}_{p} A_{2}}{p^{4}} & =\bigcup_{P} \frac{1}{2}\left[\mathcal{T}_{P}^{2}\left(\frac{1}{p^{4}}-\frac{p_{3}^{2}}{p^{6}}\right)+\left(\mathcal{T}_{P}-\mathcal{T}_{P}^{2}\right)\left(\frac{p_{0}^{2}}{p^{6}}-\frac{3 p_{0}^{2} p_{3}^{2}}{p^{8}}\right)\right] \\
& =\left[\frac{1}{2}\left\langle c_{1}^{6-d}\right\rangle_{c_{1}}\left\langle 1-3 c_{2}^{2}\right\rangle_{c_{2}}+\frac{1}{2}\left(\Delta_{4}^{\prime \prime \prime}-\Delta_{5}^{\prime \prime \prime}\right)\left\langle 1-c_{3}^{2}\right\rangle_{c_{3}}+\Delta_{5}^{\prime \prime \prime}\left\langle c_{3}^{2}\right\rangle_{c_{3}}\right] \mathcal{Y}_{P} \frac{1}{p^{2} P^{2}} \\
& =-\left(\frac{\Lambda e^{\gamma_{E}}}{4 \pi T}\right)^{2 \epsilon} \frac{2}{9(4 \pi)^{2}}\left[\frac{1+2 \ln 2}{\epsilon}+\frac{1}{60}(-91+8 \ln 2(59+15 \ln 2))\right]
\end{aligned}
$$

Here different $\Delta$ 's are the nontrivial angular averages given in Appendix C. 


\section{b. HTL sum integrals required for transverse part}

1.

$$
\begin{aligned}
\bigvee_{P} \frac{A_{1} p_{0} p_{3}}{p_{1}^{2} P^{2}} & =-\bigotimes_{P} \frac{p_{0}^{2} p_{3}^{2}}{p_{1}^{2} p^{2} P^{2}}\left(1-\mathcal{T}_{P}\right)=\left[\Delta_{3} \Delta_{0}-\Delta_{0}\right] \bigvee_{P} \frac{1}{P^{2}} \\
& =\left(\frac{\Lambda}{4 \pi T}\right)^{2 \epsilon} \frac{T^{2}}{24}\left[\frac{\ln 2-1}{\epsilon}+\frac{\pi^{2}}{6}-2+(\ln 2)^{2}+2(\ln 2-1) \frac{\zeta^{\prime}(-1)}{\zeta(-1)}\right]
\end{aligned}
$$

2.

$$
\begin{aligned}
\bigvee_{P} \frac{A_{3} p_{0} p_{3}}{p_{1}^{2} P^{2}} & =\bigcup_{P} \frac{p_{0}^{2} p_{3}^{2}}{2 p^{2} p_{1}^{2} P^{2}}\left(1-\frac{5}{3} \frac{p_{3}^{2}}{p^{2}}\right)-\bigcup_{P} \frac{3 p_{0}^{2} p_{3}^{2}\left(1-\mathcal{T}_{P}\right)}{2 p^{2} p_{1}^{2} P^{2}}\left(\frac{p_{1}^{2}}{p^{2}}-\frac{p_{0}^{2}}{p^{2}}+\frac{5}{3} \frac{p_{0}^{2}}{p^{2}} \frac{p_{3}^{2}}{p^{2}}\right) \\
& =\left[\frac{1}{2} \Delta_{0}-\frac{11}{6} \Delta_{1}+\frac{3}{2} \Delta_{3} \Delta_{0}-\frac{3}{2} \Delta_{4} \Delta_{0}-\frac{3}{2} \Delta_{3} \Delta_{1}+\frac{5}{2} \Delta_{4} \Delta_{1}\right] \bigvee_{P} \frac{1}{P^{2}} \\
& =\left(\frac{\Lambda}{4 \pi T}\right)^{2 \epsilon} \frac{T^{2}}{144}\left[\frac{6 \ln 2-5}{\epsilon}+\pi^{2}-\frac{55}{3}+2 \ln 2(3 \ln 2+5)-2(5-6 \ln 2) \frac{\zeta^{\prime}(-1)}{\zeta(-1)}\right] .
\end{aligned}
$$

3.

$$
\begin{aligned}
& \bigvee_{P} \frac{A_{2} p_{0}^{2} p_{3}^{2}}{p_{1}^{2} p^{2} P^{2}}=\bigvee_{P} \frac{1}{2}\left[\frac{\mathcal{T}_{P} p_{0}^{2} p_{3}^{2}}{p_{1}^{2} p^{2} P^{2}}\left(1-\frac{p_{3}^{2}}{p^{2}}\right)+\frac{\left(1-\mathcal{T}_{P}\right) p_{0}^{2} p_{3}^{2}}{p_{1}^{2} p^{2} P^{2}}\left(\frac{p_{0}^{2}}{p^{2}}-\frac{3 p_{0}^{2} p_{3}^{2}}{p^{4}}\right)\right]
\end{aligned}
$$

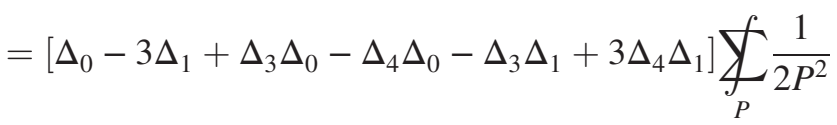

$$
\begin{aligned}
& =\left[2 \Delta_{3} \Delta_{1}+\left(1-\Delta_{7}\right)\left(\Delta_{0}-3 \Delta_{1}\right)\right] \bigotimes_{P} \frac{1}{2 P^{2}} \\
& =\left(\frac{\Lambda}{4 \pi T}\right)^{2 \epsilon} \frac{T^{2}}{48}\left[\frac{2 \ln 2-1}{\epsilon}+\frac{\pi^{2}}{3}-5+2 \ln 2\left(\ln 2+\frac{5}{3}\right)+2(2 \ln 2-1) \frac{\zeta^{\prime}(-1)}{\zeta(-1)}\right]+\mathcal{O}(\epsilon)
\end{aligned}
$$

4.

$$
\begin{aligned}
{\underset{P}{P}}_{P} \frac{A_{2}\left(5 p_{0}^{2}+9 p^{2}\right)}{4 p_{1}^{2} P^{2}} & =\bigotimes_{P} \frac{1}{2}\left[\frac{\mathcal{T}_{P}\left(5 p_{0}^{2}+9 p^{2}\right)}{4 p_{1}^{2} P^{2}}\left(1-\frac{p_{3}^{2}}{p^{2}}\right)+\frac{\left(1-\mathcal{T}_{P}\right)\left(5 p_{0}^{2}+9 p^{2}\right)}{4 p_{1}^{2} P^{2}}\left(\frac{p_{0}^{2}}{p^{2}}-\frac{3 p_{0}^{2} p_{3}^{2}}{p^{4}}\right)\right] \\
& =\left[\frac{7}{2}-7 \Delta_{0}-\frac{5}{4} \Delta_{7}-\frac{9}{4} \Delta_{9}+\frac{5}{2} \Delta_{4} \Delta_{0}+\frac{9}{2} \Delta_{3} \Delta_{0}\right] \bigcup_{P} \frac{1}{2 P^{2}} \\
& =\left(\frac{\Lambda}{4 \pi T}\right)^{2 \epsilon} \frac{7 T^{2}}{96}\left[\frac{2 \ln 2-1}{\epsilon}+\frac{\pi^{2}}{3}-\frac{79}{14}+2 \ln 2(\ln 2+1)+2(2 \ln 2-1) \frac{\zeta^{\prime}(-1)}{\zeta(-1)}\right]+\mathcal{O}(\epsilon)
\end{aligned}
$$


5.

$$
\begin{aligned}
& \bigvee_{P} \frac{A_{4}}{P^{2}}=\bigcup_{P} \frac{3}{8 P^{2}}\left(1-\frac{p_{3}^{2}}{p^{2}}\right)^{2}-\bigcup_{P} \frac{p_{0}^{2}}{8 p^{2} P^{2}}\left(1-\frac{5 p_{3}^{2}}{p^{2}}\right)^{2}+\bigcup_{P} \frac{5}{3} \frac{p_{0}^{2}}{p^{2} P^{2}} \frac{p_{3}^{4}}{p^{4}} \\
& -\frac{3}{8} y_{P}\left\{\left(1-\frac{p_{0}^{2}}{p^{2}}\right)^{2}-\frac{2 p_{3}^{2}}{p^{2}}\left(1-\frac{3 p_{0}^{2}}{p^{2}}\right)^{2}+\frac{p_{3}^{4}}{p^{4}}\left(1-\frac{5 p_{0}^{2}}{p^{2}}\right)^{2}+\frac{8 p_{0}^{4}}{p^{4}} \frac{p_{3}^{2}}{p^{2}}\left(1-\frac{5 p_{3}^{2}}{3 p^{2}}\right)\right\} \times\left(\frac{1-\mathcal{T}_{P}}{P^{2}}\right) \\
& =\frac{3}{8}\left[\frac{2}{3}+\frac{4}{3} \Delta_{0}^{\prime}-\frac{50}{9} \Delta_{1}^{\prime}+\Delta_{2}-2 \Delta_{3}+\Delta_{4}-2 \Delta_{2} \Delta_{0}^{\prime}-10 \Delta_{4} \Delta_{0}^{\prime}+12 \Delta_{3} \Delta_{0}^{\prime}+\Delta_{2} \Delta_{1}^{\prime}+\frac{35}{3} \Delta_{4} \Delta_{1}^{\prime}-10 \Delta_{3} \Delta_{1}^{\prime}\right] \sum_{P} \frac{1}{P^{2}} \\
& =\left(\frac{\Lambda}{4 \pi T}\right)^{2 \epsilon} \frac{T^{2}}{120}\left[\frac{1}{\epsilon}-\frac{13}{30}+2 \frac{\zeta^{\prime}(-1)}{\zeta(-1)}\right]
\end{aligned}
$$

6.

$$
\begin{aligned}
& \bigvee_{P} \frac{A_{1} p_{0} p_{3}\left(p_{0}^{2}-\mathcal{T}_{P} P^{2}\right)}{p_{1}^{2} p^{2} P^{4}}=-\bigvee_{P} \frac{p_{0}^{2} p_{3}^{2}\left(p_{0}^{2}-\mathcal{T}_{P} P^{2}\right)}{p_{1}^{2} p^{4} P^{4}}\left(1-\mathcal{T}_{P}\right) \\
& =-\bigvee_{P} \frac{p_{0}^{4} p_{3}^{2}}{p_{1}^{2} p^{4} P^{4}}+\bigvee_{P} \frac{p_{0}^{2} p_{3}^{2}}{p_{1}^{2} p^{4} P^{2}} \mathcal{T}_{P}+\bigvee_{P} \frac{p_{0}^{4} p_{3}^{2}}{p_{1}^{2} p^{4} P^{4}} \mathcal{T}_{P}-\bigvee_{P} \frac{p_{0}^{2} p_{3}^{2}}{p_{1}^{2} p^{4} P^{2}} \mathcal{T}_{P}^{2} \\
& =\left[\left(1+\Delta_{0}\right) \Delta_{0}-\Delta_{0}\right] \bigvee_{P} \frac{1}{P^{4}}+\left[-2 \Delta_{0}+\Delta_{4} \Delta_{0}+3\left(1+\Delta_{0}\right) \Delta_{0}-\Delta_{5}^{\prime} \Delta_{0}-\Delta_{5}^{\prime \prime} \Delta_{0}\right] \bigvee_{P} \frac{1}{p^{2} P^{2}} \\
& =\left(\frac{\Lambda e^{\gamma_{E}}}{4 \pi T}\right)^{2 \epsilon} \frac{1}{12(4 \pi)^{2}}\left[\frac{-13+\pi^{2}+4 \ln 2}{\epsilon^{2}}\right. \\
& \left.+\frac{1}{\epsilon}\left\{-\frac{137}{3}+4 \pi^{2}+\frac{4}{3} \ln 2(3 \ln 2-4)+6 \zeta(3)\right\}-0.81947\right] \text {. }
\end{aligned}
$$

7.

$$
\begin{aligned}
\bigvee_{P} \frac{A_{3} p_{0} p_{3}\left(p_{0}^{2}-\mathcal{T}_{P} P^{2}\right)}{p_{1}^{2} p^{2} P^{4}}= & \bigcup_{P} \frac{p_{0}^{2} p_{3}^{2}\left(p_{0}^{2}-\mathcal{T}_{P} P^{2}\right)}{2 p^{4} p_{1}^{2} P^{4}}\left(1-\frac{5}{3} \frac{p_{3}^{2}}{p^{2}}\right) \\
& -\bigvee_{P} \frac{3 p_{0}^{2} p_{3}^{2}\left(p_{0}^{2}-\mathcal{T}_{P} P^{2}\right)\left(1-\mathcal{T}_{P}\right)}{2 p^{4} p_{1}^{2} P^{4}}\left(1-\frac{p_{0}^{2}}{p^{2}}-\frac{p_{3}^{2}}{p^{2}}+\frac{5}{3} \frac{p_{0}^{2}}{p^{2}} \frac{p_{3}^{2}}{p^{2}}\right) \\
= & {\left[\left(1+\Delta_{0}\right) \Delta_{1}+\frac{1}{2} \Delta_{0}-\frac{11}{6} \Delta_{1}\right] Y_{P} \frac{1}{P^{4}}+\left[\frac{5}{2} \Delta_{0}-\frac{37}{6} \Delta_{1}-\frac{1}{2} \Delta_{4}\left(\Delta_{0}-\frac{5}{3} \Delta_{1}\right)\right.} \\
& -\frac{3}{2}\left(1+\Delta_{0}\right) \Delta_{0}+\frac{11}{2}\left(1+\Delta_{0}\right) \Delta_{1}-\Delta_{6}^{\prime} \Delta_{1}-\frac{3}{2}\left(\Delta_{5}^{\prime}-\Delta_{6}^{\prime}\right)\left(\Delta_{0}-\Delta_{1}\right) \\
& \left.+\frac{3}{2}\left(\Delta_{4}-\Delta_{5}\right)\left(\Delta_{0}-\Delta_{1}\right)-\frac{3}{2}\left(\Delta_{5}^{\prime \prime}-\Delta_{6}^{\prime \prime}\right)\left(\Delta_{0}-\Delta_{1}\right)+\Delta_{5} \Delta_{1}-\Delta_{6}^{\prime \prime} \Delta_{1}\right] Y_{P} \frac{1}{p^{2} P^{2}} \\
= & \left(\frac{\Lambda e^{\gamma_{E}}}{4 \pi T}\right)^{2 \epsilon} \frac{1}{60(4 \pi)^{2}}\left[\frac{-103+5 \pi^{2}+76 \ln 2}{\epsilon^{2}}+\frac{1}{15 \epsilon}\left\{-7473+550 \pi^{2}\right.\right. \\
& +12 \ln 2(118+95 \ln 2)+450 \zeta(3)\}+21.3892]+\mathcal{O}[\epsilon] .
\end{aligned}
$$


8.

$$
\begin{aligned}
\underbrace{}_{P} \frac{A_{2} p_{0}^{2} p_{3}^{2}\left(p_{0}^{2}-\mathcal{T}_{P} P^{2}\right)}{p_{1}^{2} p^{4} P^{4}}= & \bigotimes_{P} \frac{1}{2}\left[\frac{\mathcal{T}_{P} p_{0}^{2} p_{3}^{2}\left(p_{0}^{2}-\mathcal{T}_{P} P^{2}\right)}{p_{1}^{2} p^{4} P^{4}}\left(1-\frac{p_{3}^{2}}{p^{2}}-\frac{p_{0}^{2}}{p^{2}}+\frac{3 p_{0}^{2} p_{3}^{2}}{p^{4}}\right)\right. \\
& \left.+\frac{p_{0}^{2} p_{3}^{2}\left(p_{0}^{2}-\mathcal{T}_{P} P^{2}\right)}{p_{1}^{2} p^{4} P^{4}}\left(\frac{p_{0}^{2}}{p^{2}}-\frac{3 p_{0}^{2} p_{3}^{2}}{p^{4}}\right)\right] \\
= & \frac{1}{2}\left[\left(1+\Delta_{0}\right)\left(3 \Delta_{1}+\Delta_{0}^{\prime}-\Delta_{0}\right)+\Delta_{0}-3 \Delta_{1}\right] \sum_{P} \frac{1}{P^{4}}+\frac{1}{2}\left[3 \Delta_{0}-9 \Delta_{1}-\Delta_{5}\left(\Delta_{0}-3 \Delta_{1}\right)\right. \\
& +\left(1+\Delta_{0}\right)\left(12 \Delta_{1}+3 \Delta_{0}^{\prime}-4 \Delta_{0}\right)-\Delta_{5}^{\prime} \Delta_{0}^{\prime}+\Delta_{6}^{\prime}\left(\Delta_{0}-3 \Delta_{1}\right)-\Delta_{5}^{\prime \prime} \Delta_{0}^{\prime} \\
& \left.+\Delta_{6}^{\prime \prime}\left(\Delta_{0}-3 \Delta_{1}\right)\right] \bigvee_{P} \frac{1}{p^{2} P^{2}} \\
= & \left(\frac{\Lambda e^{\gamma_{E}}}{4 \pi T}\right)^{2 \epsilon} \frac{1}{60(4 \pi)^{2}}\left[\frac{-83+5 \pi^{2}+56 \ln 2}{\epsilon^{2}}+\frac{1}{15 \epsilon}\left\{-5893+500 \pi^{2}\right.\right. \\
& +\ln 2(556+840 \ln 2)+450 \zeta(3)\}+73.7496]+\mathcal{O}[\epsilon] .
\end{aligned}
$$

9.

$$
\begin{aligned}
{\underset{P}{P}}_{P} \frac{A_{2}\left(5 p_{0}^{2}+9 p^{2}\right)\left(p_{0}^{2}-\mathcal{T}_{P} P^{2}\right)}{4 p_{1}^{2} p^{2} P^{4}}= & \bigcup_{P} \frac{1}{2}\left[\frac{\mathcal{T}_{P}\left(p_{0}^{2}-\mathcal{T}_{P} P^{2}\right)\left(5 p_{0}^{2}+9 p^{2}\right)}{4 p_{1}^{2} p^{2} P^{4}}\left(1-\frac{p_{3}^{2}}{p^{2}}-\frac{p_{0}^{2}}{p^{2}}+\frac{3 p_{0}^{2} p_{3}^{2}}{p^{4}}\right)\right. \\
& \left.+\frac{\left(p_{0}^{2}-\mathcal{T}_{P} P^{2}\right)\left(5 p_{0}^{2}+9 p^{2}\right)}{4 p_{1}^{2} p^{2} P^{4}}\left(\frac{p_{0}^{2}}{p^{2}}-\frac{3 p_{0}^{2} p_{3}^{2}}{p^{4}}\right)\right] \\
= & \frac{7}{4}\left[1-2 \Delta_{0}+\left(1+\Delta_{0}\right)\left(1-\left(1+\Delta_{0}\right)+3 \Delta_{0}\right)\right] \bigvee_{P} \frac{1}{P^{4}}+\frac{1}{2}\left[\frac{33}{4}\left(1-2 \Delta_{0}\right)\right. \\
& +\frac{1}{4}\left(1+\Delta_{0}\right)\left(33-47\left(1+\Delta_{0}\right)+141 \Delta_{0}\right)-\frac{5}{4} \Delta_{5}\left(\left(1+\Delta_{0}\right)-3 \Delta_{0}\right) \\
& -\frac{9}{4} \Delta_{4}\left(\left(1+\Delta_{0}\right)-3 \Delta_{0}\right)-\frac{1}{4} \Delta_{5}^{\prime}\left(5-9\left(1+\Delta_{0}\right)+27 \Delta_{0}\right)+\frac{5}{4} \Delta_{6}^{\prime}\left(\left(1+\Delta_{0}\right)-3 \Delta_{0}\right) \\
& \left.-\frac{9}{4} \Delta_{4}^{\prime}-\frac{1}{4} \Delta_{5}^{\prime \prime}\left(5-9\left(1+\Delta_{0}\right)+27 \Delta_{0}\right)+\frac{5}{4} \Delta_{6}^{\prime \prime}\left(\left(1+\Delta_{0}\right)-3 \Delta_{0}\right)-\frac{9}{4} \Delta_{4}^{\prime \prime}\right] y_{P} \frac{1}{p^{2} P^{2}} \\
= & \left(\frac{\Lambda e^{\gamma_{E}}}{4 \pi T}\right)^{2 \epsilon} \frac{1}{24(4 \pi)^{2}}\left[\frac{-100+7 \pi^{2}+46 \ln 2}{\epsilon^{2}}+\frac{1}{15 \epsilon}\left\{-6679+495 \pi^{2}\right.\right. \\
& +2 \ln 2(599+345 \ln 2)+630 \zeta(3)\}+14.5448]+\mathcal{O}[\epsilon] .
\end{aligned}
$$

10.

$$
\begin{aligned}
& \bigcup_{P} \frac{A_{4}\left(p_{0}^{2}-\mathcal{T}_{P} P^{2}\right)}{p^{2} P^{4}}=\bigotimes_{P} \frac{3\left(p_{0}^{2}-\mathcal{T}_{P} P^{2}\right)}{8 p^{2} P^{4}}\left(1-\frac{p_{3}^{2}}{p^{2}}\right)^{2}-\bigotimes_{P} \frac{p_{0}^{2}\left(p_{0}^{2}-\mathcal{T}_{P} P^{2}\right)}{8 p^{4} P^{4}}\left(1-\frac{5 p_{3}^{2}}{p^{2}}\right)^{2} \\
& +\bigvee_{P} \frac{5}{3} \frac{p_{0}^{2}\left(p_{0}^{2}-\mathcal{T}_{P} P^{2}\right)}{p^{4} P^{4}} \frac{p_{3}^{4}}{p^{4}}-\frac{3}{8} \bigvee_{P}\left(\frac{\left(1-\mathcal{T}_{P}\right)\left(p_{0}^{2}-\mathcal{T}_{P} P^{2}\right)}{p^{2} P^{4}}\right) \\
& \times\left\{\left(1-\frac{p_{0}^{2}}{p^{2}}\right)^{2}-\frac{2 p_{3}^{2}}{p^{2}}\left(1-\frac{3 p_{0}^{2}}{p^{2}}\right)^{2}+\frac{p_{3}^{4}}{p^{4}}\left(1-\frac{5 p_{0}^{2}}{p^{2}}\right)^{2}+\frac{8 p_{0}^{4}}{p^{4}} \frac{p_{3}^{2}}{p^{2}}\left(1-\frac{5 p_{3}^{2}}{3 p^{2}}\right)\right\}
\end{aligned}
$$




$$
\begin{aligned}
& =\left[\frac{1}{4}+\frac{\Delta_{0}^{\prime}}{2}-\frac{25 \Delta_{1}^{\prime}}{12}+\left(1+\Delta_{0}\right) \Delta_{1}^{\prime}\right] \bigcup_{P} \frac{1}{P^{4}}+\left[\frac{1}{8}+\frac{19 \Delta_{0}^{\prime}}{4}-\frac{205 \Delta_{1}^{\prime}}{24}+\left(1+\Delta_{0}\right)\right. \\
& \times\left(7 \Delta_{1}^{\prime}-3 \Delta_{0}^{\prime}\right)+\Delta_{5}\left(\frac{3}{8}-\frac{15}{4} \Delta_{0}^{\prime}+\frac{35}{8} \Delta_{1}^{\prime}\right)-\Delta_{4}\left(\frac{5}{8}-\frac{13}{4} \Delta_{0}^{\prime}+\frac{55}{24} \Delta_{1}^{\prime}\right) \\
& -\frac{3}{8} \Delta_{4}^{\prime}\left(1-2 \Delta_{0}^{\prime}+\Delta_{1}^{\prime}\right)+\frac{3}{8} \Delta_{5}^{\prime}\left(2-12 \Delta_{0}^{\prime}+10 \Delta_{1}^{\prime}\right)-\frac{3}{8} \Delta_{6}^{\prime}\left(1-10 \Delta_{0}^{\prime}+\frac{35}{3} \Delta_{1}^{\prime}\right) \\
& -\frac{3}{8} \Delta_{4}^{\prime \prime}\left(1-2 \Delta_{0}^{\prime}+\Delta_{1}^{\prime}\right)+\frac{3}{8} \Delta_{5}^{\prime \prime}\left(2-12 \Delta_{0}^{\prime}+10 \Delta_{1}^{\prime}\right) \\
& \left.-\frac{3}{8} \Delta_{6}^{\prime \prime}\left(1-10 \Delta_{0}^{\prime}+\frac{35}{3} \Delta_{1}^{\prime}\right)\right] y_{P} \frac{1}{p^{2} P^{2}} \\
& =\left(\frac{\Lambda e^{\gamma_{E}}}{4 \pi T}\right)^{2 \epsilon} \frac{1}{30(4 \pi)^{2}}\left[\frac{3-\pi^{2}+12 \ln 2}{\epsilon}+25-\frac{16 \pi^{2}}{15}+\frac{12 \ln 2}{5}(5 \ln 2-7)-6 \zeta(3)\right] .
\end{aligned}
$$

\section{APPENDIX C: $c$ INTEGRATIONS}

In this section we note down the following angular averages, which appeared throughout this paper due to the angular integrals $A_{n}\left(A_{0} \equiv \mathcal{T}_{P}\right)$. The symbol \langle\rangle$_{c}$ depicts the standard definition given in Ref. [78].

$$
\begin{aligned}
& \Delta_{0}=\left\langle\frac{c^{2}}{1-c^{2}}\right\rangle_{c}=-\frac{1}{2 \epsilon}+\mathcal{O}[\epsilon]^{3} \\
& \Delta_{0}^{\prime}=\left\langle c^{2}\right\rangle_{c}=\frac{1}{3}+\frac{2 \epsilon}{9}+\frac{4 \epsilon^{2}}{27}+\mathcal{O}[\epsilon]^{3}, \\
& \Delta_{0}^{\prime \prime}=\left\langle\frac{1}{\left(1-c_{1}^{2}\right)\left(1-c_{2}^{2}\right)}\right\rangle_{c_{1}, c_{2}}=\frac{(d-2)^{2}}{(d-3)^{2}}=\frac{1}{4 \epsilon^{2}}-\frac{1}{\epsilon}+1 \\
& \Delta_{1}=\left\langle\frac{c^{4}}{1-c^{2}}\right\rangle_{c}=-\frac{1}{2 \epsilon}-\frac{1}{3}-\frac{2 \epsilon}{9}-\frac{4 \epsilon^{2}}{27}+\mathcal{O}[\epsilon]^{3}, \\
& \Delta_{1}^{\prime}=\left\langle c^{4}\right\rangle_{c}=\frac{1}{5}+\frac{16 \epsilon}{75}+\mathcal{O}[\epsilon]^{2} \\
& \Delta_{2}=\left\langle\frac{1-c^{2-d}}{1-c^{2}}\right\rangle_{c}=1-\frac{1}{2 \epsilon} \\
& \Delta_{3}=\left\langle\frac{1-c^{4-d}}{1-c^{2}}\right\rangle_{c}=\ln 2+\left(\frac{\pi^{2}}{6}-(2-\ln 2) \ln 2\right) \epsilon+\left\{\frac{2}{3}(\ln 2)^{2}(\ln 2-3)+\frac{\pi^{2}}{3}(\ln 2-1)+\zeta(3)\right\} \epsilon^{2}+\mathcal{O}[\epsilon]^{3}, \\
& \Delta_{3}^{\prime}=\left\langle\frac{1-c^{4-d}}{\left(1-c^{2}\right)^{2}}\right\rangle_{c}=-\frac{1}{4 \epsilon}+\frac{1}{4}+\frac{3 \epsilon}{4}-\frac{3 \epsilon^{2}}{4}+\mathcal{O}[\epsilon]^{3}, \\
& \Delta_{3}^{\prime \prime}=\left\langle\frac{1-c_{1}^{4-d}}{\left(1-c_{1}^{2}\right)\left(c_{1}^{2}-c_{2}^{2}\right)}+c_{1} \leftrightarrow c_{2}\right\rangle_{c_{1}, c_{2}}=-\frac{\pi^{2}}{12}+\left(\frac{\pi^{2}}{3}-\frac{\zeta(3)}{2}\right) \epsilon+\mathcal{O}\left(\epsilon^{2}\right),
\end{aligned}
$$




$$
\begin{aligned}
& \Delta_{4}=\left\langle\frac{1-c^{6-d}}{1-c^{2}}\right\rangle_{c}=\left(\frac{1}{2}+\ln 2\right)+\left(\frac{\pi^{2}}{6}-1-(1-\ln 2) \ln 2\right) \epsilon \\
& +\epsilon^{2}\left(\zeta(3)+\frac{1}{3} \ln 2\{\ln 2(2 \ln 2-3)-6\}+\frac{1}{6} \pi^{2}(2 \ln 2-1)\right)+\mathcal{O}[\epsilon]^{3}, \\
& \Delta_{4}^{\prime}=\left\langle\frac{1-c^{6-d}}{\left(1-c^{2}\right)^{2}}\right\rangle_{c}=-\frac{3}{4 \epsilon}+\frac{5}{4}-\ln 2+\epsilon\left(\frac{3}{4}-\frac{\pi^{2}}{6}-\ln ^{2}(2)+\ln 4\right)+\frac{1}{12} \epsilon^{2}(-12 \zeta(3) \\
& \left.-9-\ln ^{3}(4)+6 \ln ^{2}(4)-2 \pi^{2}(\ln 4-2)\right)+\mathcal{O}[\epsilon]^{3}, \\
& \Delta_{4}^{\prime \prime}=\left\langle\frac{1-c_{1}^{6-d}}{\left(1-c_{1}^{2}\right)\left(c_{1}^{2}-c_{2}^{2}\right)}+c_{1} \leftrightarrow c_{2}\right\rangle_{c_{1}, c_{2}}=-\frac{\pi^{2}}{12}+\ln 4+\left(\frac{\pi^{2}}{3}-\ln 4(2-\ln 2)-\frac{\zeta(3)}{2}\right) \epsilon+\mathcal{O}\left(\epsilon^{2}\right), \\
& \Delta_{4}^{\prime \prime \prime}=\left\langle\frac{c_{1}^{6-d}-c_{2}^{6-d}}{c_{1}^{2}-c_{2}^{2}}\right\rangle_{c_{1}, c_{2}}=\frac{1}{3}(1+2 \ln 2)+\frac{2}{9}(-5+\ln 2(5+3 \ln 2)) \epsilon+\mathcal{O}[\epsilon]^{2}, \\
& \Delta_{5}=\left\langle\frac{1-c^{8-d}}{1-c^{2}}\right\rangle_{c}=\frac{3}{4}+\ln 2+\frac{\epsilon}{12}\left(2 \pi^{2}+3((\ln (4)-1) \ln (4)-5)\right) \\
& +\left(\zeta(3)-\frac{1}{2}-\frac{\pi^{2}}{12}+\frac{\ln 2}{6}\left(2 \pi^{2}-15+4 \ln ^{2} 2-3 \ln 2\right)\right) \epsilon^{2}, \\
& \Delta_{5}^{\prime}=\left\langle\frac{1-c^{8-d}}{\left(1-c^{2}\right)^{2}}\right\rangle_{c}=-\frac{5}{4 \epsilon}+\frac{7}{4}-\ln 4+\epsilon\left(\frac{7}{4}-\frac{\pi^{2}}{3}-\frac{\ln ^{2}(4)}{2}+\ln 8\right)+\frac{\epsilon^{2}}{12}(-24 \zeta(3) \\
& \left.-9+\pi^{2}(6-4 \ln 4)+\ln 4(12+(9-2 \ln 4) \ln 4)\right)+\mathcal{O}[\epsilon]^{3} \text {, } \\
& \Delta_{5}^{\prime \prime}=\left\langle\frac{1-c_{1}^{8-d}}{\left(1-c_{1}^{2}\right)\left(c_{1}^{2}-c_{2}^{2}\right)}+c_{1} \leftrightarrow c_{2}\right\rangle_{c_{1}, c_{2}}=\frac{1}{12}\left(4-\pi^{2}+32 \ln 2\right) \\
& +\frac{1}{18}\left(-20+6 \pi^{2}-52 \ln 2+48(\ln 2)^{2}-9 \zeta(3)\right) \epsilon+0.469927 \epsilon^{2}+\mathcal{O}[\epsilon]^{3}, \\
& \Delta_{5}^{\prime \prime \prime}=\left\langle\frac{c_{1}^{8-d}-c_{2}^{8-d}}{c_{1}^{2}-c_{2}^{2}}\right\rangle_{c_{1}, c_{2}}=\frac{1}{10}(3+\ln 16)+\frac{1}{150}(-107+2 \ln 2(97+30 \ln 2)) \epsilon+\mathcal{O}[\epsilon]^{2}, \\
& \Delta_{6}^{\prime}=\left\langle\frac{1-c^{10-d}}{\left(1-c^{2}\right)^{2}}\right\rangle_{c}=2-\frac{7}{4 \epsilon}+\frac{1}{4} \epsilon\left(12-2 \pi^{2}+(7-3 \ln 4) \ln 4\right)-\ln 8+\frac{1}{24} \epsilon^{2}(-72 \zeta(3) \\
& \left.-6+2 \pi^{2}(7-6 \ln 4)+3 \ln 4(18+(7-2 \ln 4) \ln 4)\right)+\mathcal{O}[\epsilon]^{3}
\end{aligned}
$$

$$
\begin{aligned}
\Delta_{6}^{\prime \prime} & =\left\langle\frac{1-c_{1}^{10-d}}{\left(1-c_{1}^{2}\right)\left(c_{1}^{2}-c_{2}^{2}\right)}+c_{1} \leftrightarrow c_{2}\right\rangle_{c_{1}, c_{2}} \\
& =\frac{1}{60}\left(38-5 \pi^{2}+184 \ln 2\right)+\frac{1}{450}\left(-821+150 \pi^{2}+(-359+690 \ln 2) 2 \ln 2-225 \zeta(3)\right) \epsilon+1.04576 \epsilon^{2}+\mathcal{O}[\epsilon]^{3},
\end{aligned}
$$$$
\Delta_{7}=\left\langle c^{2 \epsilon+1}\right\rangle_{c}=\frac{1}{2}+\mathcal{O}[\epsilon],
$$$$
\Delta_{8}=\left\langle c^{2 \epsilon+3}\right\rangle_{c}=\frac{1}{4}+\mathcal{O}[\epsilon],
$$

$$
\Delta_{9}=\left\langle c^{2 \epsilon-1}\right\rangle_{c}=\frac{1}{2 \epsilon}-1+\ln 2+\mathcal{O}[\epsilon],
$$




$$
\Delta_{10}=\left\langle\frac{c_{1}^{3+2 \epsilon}-c_{1}^{2}}{\left(c_{1}^{2}-c_{2}^{2}\right)\left(1-c_{1}^{2}\right)^{2}}-\frac{c_{2}^{3+2 \epsilon}-c_{2}^{2}}{\left(c_{1}^{2}-c_{2}^{2}\right)\left(1-c_{2}^{2}\right)^{2}}\right\rangle_{c_{1}, c_{2}}
$$

Unlike other $\Delta_{i}$ functions, computation of $\Delta_{10}$ is not straightforward and cannot be done directly analytically in Mathematica in $\epsilon \rightarrow 0$. After calculating the angular average in Eq. (C23), we end up with the following equation:

$$
\begin{aligned}
\Delta_{10}= & -\frac{1}{\pi \Gamma(1-\epsilon)^{2}} \Gamma(-1-\epsilon)^{2} \Gamma(3 / 2-\epsilon)^{2} \times\left[\frac { 1 } { 3 } 4 ^ { - 1 - \epsilon } e ^ { - i \pi \epsilon } ( 1 + 3 \epsilon + 2 \epsilon ^ { 2 } ) \Gamma ( 1 + 2 \epsilon ) \left\{3 e^{2 i \pi \epsilon} F\left(\begin{array}{c}
\epsilon, \frac{3}{2}+\epsilon \mid 1 \\
\frac{1}{2}
\end{array}\right)\right.\right. \\
& +\left(2+e^{2 i \pi \epsilon}\right)\left(3 F\left(\begin{array}{c}
\frac{3}{2}+\epsilon, 2+\epsilon \\
\frac{1}{2}
\end{array} \mid 1\right)-6(3+2 \epsilon) F\left(\begin{array}{c}
2+\epsilon, \frac{5}{2}+\epsilon \\
\frac{3}{2}
\end{array}\right)\right. \\
& \left.\left.+(5+2 \epsilon)(3+2 \epsilon) F\left(\begin{array}{c}
2+\epsilon, \frac{7}{2}+\epsilon \\
\frac{5}{2}
\end{array} \mid 1\right]\right)\right\}-\frac{\pi}{\Gamma[-1 / 2-\epsilon]^{2}}\left\{-F\left(\begin{array}{c}
1, \frac{3}{2}+\epsilon \\
-\frac{1}{2}-\epsilon
\end{array}\right) 1\right) \\
& \left.\left.-\frac{3+2 \epsilon}{1+2 \epsilon} F\left(\begin{array}{c}
1, \frac{5}{2}+\epsilon \\
\frac{1}{2}-\epsilon
\end{array} \mid 1\right)+\frac{1}{(1+2 \epsilon)^{2}} F\left(\begin{array}{c}
1, \frac{3}{2}, \frac{1}{2}+\epsilon \\
-\frac{1}{2}, \frac{1}{2}-\epsilon
\end{array}\right)+\frac{3}{1-4 \epsilon^{2}} F\left(\begin{array}{c}
1, \frac{5}{2}, \frac{3}{2}+\epsilon \\
\frac{1}{2}, \frac{3}{2}-\epsilon
\end{array}\right)\right\}\right] .
\end{aligned}
$$

Equation (C24) cannot be expanded directly at small $\epsilon$ in Mathematica. So, we use the following technique to expand Eq. (C24) at small $\epsilon$. In Eq. (C24), $F$ represents the generalized hypergeometric function. The generalized hypergeometric function of type ${ }_{p} F_{q}$ is an analytic function of one variable with $p+q$ parameters. Here, the parameters are functions of $\epsilon$, so the list of parameters sometimes gets so lengthy and the standard notation for these functions becomes cumbersome. We therefore introduce a more compact notation as

$$
F\left(\begin{array}{c}
\alpha_{1}, \alpha_{2}, \cdots \alpha_{n} \\
\beta_{1}, \cdots \beta_{n-1}
\end{array} \mid 1\right)=F_{q}\left(\alpha_{1}, \alpha_{2}, \cdots \alpha_{p} ; \beta_{1}, \cdots \beta_{q} ; z\right) .
$$

It is not possible to directly expand ${ }_{p} F_{q}$ at small $\epsilon$. So, we will use the following procedure to expand ${ }_{p} F_{q}$ in the series of $\epsilon$.

In Eq. (C24), there are two types of hypergeometric function viz., ${ }_{2} F_{1}$ and ${ }_{3} F_{2 \cdot}{ }_{2} F_{1}$ can be expanded in small $\epsilon$ if one uses the following relation:

$$
F\left(\alpha_{1}, \alpha_{2} ; \beta_{1} ; 1\right)=\frac{\Gamma\left(\beta_{1}\right) \Gamma\left(\beta_{1}-\alpha_{1}-\alpha_{2}\right)}{\Gamma\left(\beta_{1}-\alpha_{1}\right) \Gamma\left(\beta_{1}-\alpha_{2}\right)} .
$$

To expand ${ }_{3} F_{2}$ type of hypergeometric function, we can try the following power series representation for the generalized hypergeometric function as

$$
F\left(\begin{array}{c}
\alpha_{1}, \alpha_{2}, \ldots, \alpha_{p} \\
\beta_{1}, \ldots, \beta_{q}
\end{array} \mid z\right)=\sum_{n=0}^{\infty} \frac{\left(\alpha_{1}\right)_{n}\left(\alpha_{2}\right)_{n} \cdots\left(\alpha_{p}\right)_{n}}{\left(\beta_{1}\right)_{n} \cdots\left(\beta_{q}\right)_{n} n !} z^{n}
$$

where $(a)_{b}$ is Pochhammer's symbol:

$$
(a)_{b}=\frac{\Gamma(a+b)}{\Gamma(a)} .
$$

The power series converges for $|z|<1$. For $z=1$, it converges if $\operatorname{Re} s>0$, where

$$
s=\sum_{i=1}^{p-1} \beta_{i}-\sum_{i=1}^{p} \alpha_{i}
$$

In Eq. (C24), both ${ }_{3} F_{2}$ have negative $s$ value for $\epsilon \rightarrow 0$. So, we will use the following relation to change the parameters to make $s$ value positive: 


$$
F\left(\begin{array}{c|c}
\alpha_{1}, \alpha_{2}, \alpha_{3} \\
\beta_{1}, \beta_{2}
\end{array} \mid 1\right)=\frac{\Gamma\left(\beta_{1}\right) \Gamma\left(\beta_{2}\right) \Gamma(s)}{\Gamma\left(\alpha_{1}+s\right) \Gamma\left(\alpha_{2}+s\right) \Gamma\left(\alpha_{3}\right)} F\left(\begin{array}{c}
\beta_{1}-\alpha_{3}, \beta_{2}-\alpha_{3}, s \\
\alpha_{1}+s, \alpha_{2}+s
\end{array}\right)
$$

\section{Expansion of ${ }_{3} F_{2}\left(1, \frac{3}{2}, \frac{1}{2}+\epsilon ;-\frac{1}{2}, \frac{1}{2}-\epsilon ; 1\right)$}

Here, $s=\beta_{1}+\beta_{2}-\alpha_{1}-\alpha_{2}-\alpha_{3}=-3-2 \epsilon<0$ at $\epsilon \rightarrow 0$. So, we use Eq. (C30) to change the parameter,

$$
F\left(\begin{array}{c}
1, \frac{3}{2}, \frac{1}{2}+\epsilon \\
-\frac{1}{2}, \frac{1}{2}-\epsilon
\end{array} \mid 1\right)=\frac{\Gamma\left(-\frac{1}{2}\right) \Gamma\left(\frac{1}{2}-\epsilon\right) \Gamma(3-2 \epsilon)}{\Gamma(-2-2 \epsilon) \Gamma\left(-\frac{3}{2}-2 \epsilon\right) \Gamma\left(\frac{1}{2}+\epsilon\right)} F\left(\begin{array}{c}
-1-\epsilon,-2 \epsilon,-3-2 \epsilon \\
-2-2 \epsilon,-\frac{3}{2}-2 \epsilon
\end{array} \mid 1\right)
$$

For the hypergeometric function that appears on the right-hand side of the above equation, $s=1 / 2+9 \epsilon>0$ at $\epsilon \rightarrow 0$, so the power series expansion will converge.

So,

$$
F\left(\begin{array}{c}
-1-\epsilon,-2 \epsilon,-3-2 \epsilon \\
-2+2 \epsilon,-\frac{3}{2}+2 \epsilon
\end{array} \mid 1\right)=\sum_{n=0}^{\infty} \frac{\Gamma(-1-\epsilon+n) \Gamma(-2 \epsilon+n) \Gamma(-3-2 \epsilon+n) \Gamma(-2-2 \epsilon) \Gamma\left(-\frac{3}{2}-2 \epsilon\right)}{\Gamma(-2-2 \epsilon+n) \Gamma\left(-\frac{3}{2}-2 \epsilon+n\right) \Gamma(-1-\epsilon) \Gamma(-2 \epsilon) \Gamma(-3-2 \epsilon)} \frac{1}{n !}
$$

In the above sum, if one expands the term within summation at small $\epsilon$, one ends up with an expression that diverges for $n=0$ to $n=3$. To avoid that we will do the summation before the expansion from $n=0$ to $n=3$. From $n=4$, we will perform the summation after expansion in small $\epsilon$.

So,

$$
\begin{aligned}
{ }_{3} F_{2}( & \left.-1-\epsilon,-2 \epsilon,-3-2 \epsilon ;-2+2 \epsilon,-\frac{3}{2}+2 \epsilon ; 1\right) \\
= & \left(\sum_{n=0}^{3}+\sum_{n=4}^{\infty}\right) \frac{\Gamma(-1-\epsilon+n) \Gamma(-2 \epsilon+n) \Gamma(-3-2 \epsilon+n) \Gamma(-2-2 \epsilon) \Gamma\left(-\frac{3}{2}-2 \epsilon\right)}{\Gamma(-2-2 \epsilon+n) \Gamma\left(-\frac{3}{2}-2 \epsilon+n\right) \Gamma(-1-\epsilon) \Gamma(-2 \epsilon) \Gamma(-3-2 \epsilon)} \frac{1}{n !} \\
= & {\left[1-\frac{14}{3} \epsilon+\frac{64}{9} \epsilon^{2}-\frac{652}{27} \epsilon^{3}+\mathcal{O}\left(\epsilon^{4}\right)\right]+\sum_{n=4}^{\infty}\left[\frac{8 \sqrt{\pi} \Gamma(n-1)}{n(n-3) \Gamma\left(n-\frac{3}{2}\right)} \epsilon^{2}\right.} \\
& \left.-\frac{8 \sqrt{\pi} \Gamma(n)\left(3 \gamma_{E}+11+\frac{9}{n-3}+\frac{9}{n-2}+\frac{6}{n-1}+15 \psi(0)(n-3)-12 \psi^{(0)}(2 n-4)\right) \epsilon^{3}}{3 n(n-3)(n-1) \Gamma\left(n-\frac{3}{2}\right)}+\mathcal{O}\left(\epsilon^{4}\right)\right] \\
= & 1-\frac{14}{3} \epsilon+\frac{64}{9} \epsilon^{2}-\frac{652}{27} \epsilon^{3}+\frac{16}{3}{ }_{4} F_{3}\left(1,1,3,4 ; 2,5, \frac{5}{2} ; 1\right) \epsilon^{2} \\
& +\frac{1}{18}\left[32\left(3 \gamma_{E}-11+6 \ln 2\right)_{3} F_{2}\left(1,1,4 ; \frac{5}{2}, 5 ; 1\right)+32\left(6 \gamma_{E}-11+12 \ln 2\right){ }_{3} F_{2}\left(1,1,3 ; \frac{3}{2}, 4 ; 1\right)\right. \\
& \left.+252 \zeta(3)-36 \gamma_{E} \pi^{2}-51 \pi^{2}-640 \gamma_{E}+824-72 \pi^{2} \ln 2-1280 \ln 2\right] \epsilon^{3}+\mathcal{O}\left(\epsilon^{4}\right) .
\end{aligned}
$$

The hypergeometric function of type ${ }_{p+1} F_{q+1}$ has an integral representation in terms of the hypergeometric function of type ${ }_{p} F_{q}$ :

$$
\int_{0}^{1} d t t^{\nu-1}(1-t)^{\mu-1} F\left(\begin{array}{c}
\alpha_{1}, \alpha_{2}, \ldots, \alpha_{p} \\
\beta_{1}, \ldots, \beta_{q}
\end{array} \mid t z\right)=\frac{\Gamma(\mu) \Gamma(\nu)}{\Gamma(\mu+\nu)} F\left(\begin{array}{c}
\alpha_{1}, \alpha_{2}, \ldots, \alpha_{p}, \nu \\
\beta_{1}, \ldots, \beta_{q}, \mu+\nu
\end{array} \mid z\right)
$$

Using Eq. (C34), Eq. (C33) can be simplified as 


$$
\begin{aligned}
{ }_{3} F_{2}( & \left.-1-\epsilon,-2 \epsilon,-3-2 \epsilon ;-2+2 \epsilon,-\frac{3}{2}+2 \epsilon ; 1\right) \\
= & 1-\frac{14}{3} \epsilon+\frac{64}{9} \epsilon^{2}-\frac{652}{27} \epsilon^{3}+\frac{16}{3} \int_{0}^{1} d t F\left(\begin{array}{c}
1,3,4 \\
5, \frac{5}{2}
\end{array} \mid t\right) \epsilon^{2} \\
& +\frac{1}{18}\left[32\left(3 \gamma_{E}-11+6 \ln 2\right) \times 3 \int_{0}^{1} d t t^{2}{ }_{2} F_{1}\left(1,1 ; \frac{3}{2} ; t\right)\right. \\
& +32\left(6 \gamma_{E}-11+12 \ln 2\right) \times 4 \int_{0}^{1} d t t^{3}{ }_{2} F_{1}\left(1,1 ; \frac{5}{2} ; t\right) \\
& \left.+252 \zeta(3)+824-51 \pi^{2}-4\left(160+9 \pi^{2}\right)\left(\gamma_{E}+2 \ln 2\right)\right] \epsilon^{3}+\mathcal{O}\left(\epsilon^{4}\right) \\
= & 1-\frac{14}{3} \epsilon+\frac{64}{9} \epsilon^{2}-\frac{652}{27} \epsilon^{3}+\left(\pi^{2}+\frac{112}{9}\right) \epsilon^{2}+\left(14 \zeta(3)-\frac{2}{27}\left(746+63 \pi^{2}\right)\right) \epsilon^{3}+\mathcal{O}\left(\epsilon^{4}\right) \\
= & 1-\frac{14}{3} \epsilon+\left(\pi^{2}+\frac{176}{9}\right) \epsilon^{2}+\left(-\frac{2144}{27}-\frac{14 \pi^{2}}{3}+14 \zeta(3)\right) \epsilon^{3}+\mathcal{O}\left(\epsilon^{4}\right) .
\end{aligned}
$$

So, Eq. (C31) can be rewritten as

$$
\begin{aligned}
F\left(1, \frac{3}{2}, \frac{1}{2}+\epsilon ;-\frac{1}{2}, \frac{1}{2}-\epsilon ; 1\right)= & \frac{\Gamma\left(-\frac{1}{2}\right) \Gamma\left(\frac{1}{2}-\epsilon\right) \Gamma(3-2 \epsilon)}{\Gamma(-2-2 \epsilon) \Gamma\left(-\frac{3}{2}-2 \epsilon\right) \Gamma\left(\frac{1}{2}+\epsilon\right)} \\
& \times\left[1-\frac{14}{3} \epsilon+\left(\pi^{2}+\frac{176}{9}\right) \epsilon^{2}+\left(-\frac{2144}{27}-\frac{14 \pi^{2}}{3}+14 \zeta(3)\right) \epsilon^{3}+\mathcal{O}\left(\epsilon^{4}\right)\right] .
\end{aligned}
$$

\section{Expansion of ${ }_{3} F_{2}\left(1, \frac{5}{2}, \frac{3}{2}+\epsilon ; \frac{1}{2}, \frac{3}{2}-\epsilon ; 1\right)$}

Following a similar procedure, we can write

$$
\begin{aligned}
{ }_{3} F_{2}\left(1, \frac{5}{2}, \frac{3}{2}+\epsilon ; \frac{1}{2}, \frac{3}{2}-\epsilon ; 1\right)= & \frac{\Gamma\left(\frac{1}{2}\right) \Gamma\left(\frac{3}{2}-\epsilon\right) \Gamma(3-2 \epsilon)}{\Gamma(-2-2 \epsilon) \Gamma\left(-\frac{1}{2}-2 \epsilon\right) \Gamma\left(\frac{3}{2}+\epsilon\right)} \\
& \times\left[1-\frac{10}{3} \epsilon+\left(\pi^{2}+\frac{40}{3}\right) \epsilon^{2}+\left(-\frac{160}{3}-\frac{10 \pi^{2}}{3}+14 \zeta(3)\right) \epsilon^{3}+\mathcal{O}\left(\epsilon^{4}\right)\right] .
\end{aligned}
$$

Adding all the contributions, we can write Eq. (C24) as

$$
\begin{aligned}
\Delta_{10} & =\left\langle\frac{c_{1}^{3+2 \epsilon}-c_{1}^{2}}{\left(c_{1}^{2}-c_{2}^{2}\right)\left(1-c_{1}^{2}\right)^{2}}-\frac{c_{2}^{3+2 \epsilon}-c_{2}^{2}}{\left(c_{1}^{2}-c_{2}^{2}\right)\left(1-c_{2}^{2}\right)^{2}}\right\rangle_{c_{1}, c_{2}} \\
& =-\frac{1}{8 \epsilon^{2}}+\frac{1}{4 \epsilon}+\frac{1}{24}\left(18-\pi^{2}\right)+\frac{1}{12}\left(2 \pi^{2}-3(9+\zeta(3))\right) \epsilon+\mathcal{O}\left(\epsilon^{2}\right) .
\end{aligned}
$$

The remaining $c$ integration $\Delta_{11}$ can be written as

$$
\begin{aligned}
\Delta_{11} & =\left\langle\frac{c_{1}^{1+2 \epsilon}-c_{1}^{2}}{\left(c_{1}^{2}-c_{2}^{2}\right)\left(1-c_{1}^{2}\right)^{2}}-\frac{c_{2}^{1+2 \epsilon}-c_{2}^{2}}{\left(c_{1}^{2}-c_{2}^{2}\right)\left(1-c_{2}^{2}\right)^{2}}\right\rangle_{c_{1}, c_{2}} \\
& =\Delta_{0}^{\prime \prime}+\Delta_{10}-\Delta_{3}^{\prime \prime} \\
& =\frac{1}{8 \epsilon^{2}}-\frac{3}{4 \epsilon}+\frac{1}{24}\left(\pi^{2}+42\right)+\frac{1}{12}\left(3(\zeta(3)-9)-2 \pi^{2}\right) \epsilon+\mathcal{O}\left(\epsilon^{2}\right) .
\end{aligned}
$$




\section{APPENDIX D: FREE ENERGY WITHOUT HIGH $T$ EXPANSION}

\section{Quark part}

Free energy of a quark is given by

$$
F_{q}=-N_{c} \sum_{f} \int \frac{d^{4} P}{(2 \pi)^{4}} \ln \left(\operatorname{det}\left[S_{\mathrm{eff}}^{-1}(P)\right]\right)
$$

Argument of the logarithm can also be factorized as

$$
\begin{aligned}
\operatorname{det}\left[S_{\text {eff }}^{-1}\right]= & \left(\mathcal{C}^{2} p_{0}^{2}-\mathcal{D}^{2} p^{2}+\mathcal{B}^{\prime 2}-\mathcal{C}^{\prime 2}\right)^{2}-4\left(p_{0} \mathcal{B}^{\prime} \mathcal{C}+p_{3} \mathcal{C}^{\prime} \mathcal{D}\right)^{2} \\
= & {\left[\left(\mathcal{C} p_{0}-\mathcal{B}^{\prime}\right)^{2}-\left(\left(\mathcal{D} p_{3}+\mathcal{C}^{\prime}\right)^{2}+\mathcal{D}^{2} p_{\perp}^{2}\right)\right] \times\left[\left(\mathcal{C} p_{0}+\mathcal{B}^{\prime}\right)^{2}-\left(\left(\mathcal{D} p_{3}-\mathcal{C}^{\prime}\right)^{2}+\mathcal{D}^{2} p_{\perp}^{2}\right)\right] } \\
= & {\left[\left((1+\mathcal{A}) p_{0}+\mathcal{B}-\mathcal{B}^{\prime}\right)^{2}-\left(\left((1+\mathcal{A}) p_{3}+\mathcal{C}^{\prime}\right)^{2}+(1+\mathcal{A})^{2} p_{\perp}^{2}\right)\right] } \\
& \times\left[\left((1+\mathcal{A}) p_{0}+\mathcal{B}+\mathcal{B}^{\prime}\right)^{2}-\left(\left((1+\mathcal{A}) p_{3}-\mathcal{C}^{\prime}\right)^{2}+(1+\mathcal{A})^{2} p_{\perp}^{2}\right)\right] \\
= & L_{+} L_{-} R_{+} R_{-},
\end{aligned}
$$

with

$$
\begin{aligned}
& L_{ \pm}=\left((1+\mathcal{A}) p_{0}+\mathcal{B}-\mathcal{B}^{\prime}\right) \mp \sqrt{\left((1+\mathcal{A}) p_{3}+\mathcal{C}^{\prime}\right)^{2}+(1+\mathcal{A})^{2} p_{\perp}^{2}}, \\
& R_{ \pm}=\left((1+\mathcal{A}) p_{0}+\mathcal{B}+\mathcal{B}^{\prime}\right) \mp \sqrt{\left((1+\mathcal{A}) p_{3}-\mathcal{C}^{\prime}\right)^{2}+(1+\mathcal{A})^{2} p_{\perp}^{2}},
\end{aligned}
$$

and

$$
\begin{aligned}
\mathcal{A}\left(p_{0}, p\right) & =-\frac{m_{\mathrm{th}}^{2}}{p^{2}} Q_{1}\left(\frac{p_{0}}{p}\right), \\
\mathcal{B}\left(p_{0}, p\right) & =\frac{m_{\mathrm{th}}}{p}\left[\frac{p_{0}}{p} Q_{1}\left(\frac{p_{0}}{p}\right)-Q_{0}\left(\frac{p_{0}}{p}\right)\right], \\
\mathcal{B}^{\prime}\left(p_{0}, p, \theta\right) & =-\frac{m_{\mathrm{eff}}^{2} p_{z}}{p^{2}} Q_{1}\left(\frac{p_{0}}{p}\right), \\
\mathcal{C}^{\prime}\left(p_{0}, p\right) & =\frac{m_{\mathrm{eff}}^{2}}{p} Q_{0}\left(\frac{p_{0}}{p}\right) .
\end{aligned}
$$

Using these in Eq. (D1) one gets

$$
\begin{aligned}
F_{\mathrm{q}} & =-N_{c} \sum_{f} \int \frac{d^{4} P}{(2 \pi)^{4}} \ln L_{+} L_{-} R_{+} R_{-} \\
& =-N_{c} N_{f} \int \frac{d^{4} P}{(2 \pi)^{4}} \ln P^{4}-N_{c} \sum_{f} \int \frac{d^{4} P}{(2 \pi)^{4}} \ln \frac{L_{+} L_{-} R_{+} R_{-}}{P^{4}} \\
& =F_{\text {Free }}^{q}+F_{\mathrm{QP}}^{q}+F_{\mathrm{LD}}^{q},
\end{aligned}
$$

where the free part is obtained as

$$
F_{\text {Free }}^{q}=-N_{c} N_{f} \frac{7 \pi^{2} T^{4}}{180}
$$


The quasiparticle $(\mathrm{QP})$ part $F_{\mathrm{QP}}$ is given as

$$
\begin{aligned}
F_{\mathrm{QP}}^{q} & =-N_{c} \sum_{f} \int \frac{d^{3} p}{(2 \pi)^{3}} \oint \frac{d p_{0}}{4 \pi i} \ln \frac{D_{+} D_{-}}{P^{4}} \tanh \frac{\beta p_{0}}{2} \\
& =-N_{c} \sum_{f} \int \frac{d^{3} p}{(2 \pi)^{3}} \oint \frac{d p_{0}}{4 \pi i}\left[\ln L_{+}+\ln L_{-}+\ln R_{+}+\ln R_{-}-\ln P^{4}\right] \tanh \frac{\beta p_{0}}{2} \\
& =-N_{c} \sum_{f} \int \frac{d^{3} p}{(2 \pi)^{3}}\left[I_{1}+I_{2}+I_{3}+I_{4}-I_{0}\right] .
\end{aligned}
$$

One has the following dispersion solutions for a quark in a thermomagnetic medium:

$L_{+}$has solutions at $p_{0}=\omega_{L}^{+},-\omega_{R}^{-}$,

$L_{-}$has solutions at $p_{0}=\omega_{L}^{-},-\omega_{R}^{+}$,

$R_{+}$has solutions at $p_{0}=\omega_{R}^{+},-\omega_{L}^{-}$,

$R_{-}$has solutions at $p_{0}=\omega_{R}^{-},-\omega_{L}^{+}$.

Now one can calculate the various integrations in Eq. (D6) as

$$
\begin{aligned}
I_{1} & =\oint \frac{d p_{0}}{4 \pi i} \ln L_{+} \tanh \frac{\beta p_{0}}{2} \\
& =-\frac{2}{\beta} \oint \frac{d p_{0}}{4 \pi i} \frac{L_{+}^{\prime}}{L_{+}} \ln \cosh \frac{\beta p_{0}}{2} \\
& =\frac{T}{-2 \pi i} \oint d p_{0} \frac{L_{+}^{\prime}}{L_{+}} \ln \cosh \frac{\beta p_{0}}{2} .
\end{aligned}
$$

The integral has poles at $p_{0}=\omega_{L}^{+},-\omega_{R}^{-}$. We now calculate the residues as

$$
\begin{aligned}
\left.R\right|_{p_{0}= \pm \omega_{i}} & =\left.\frac{L_{+}^{\prime}\left(p_{0}, p\right)}{L_{+}\left( \pm \omega_{i}, p\right)+\left(p_{0} \mp \omega_{i}\right) L_{+}^{\prime}\left( \pm \omega_{i}, p\right)} \ln \cosh \frac{\beta p_{0}}{2}\right|_{p_{0}= \pm \omega_{i}} \\
& =\ln \cosh \frac{ \pm \beta \omega_{i}}{2}=\frac{\beta \omega_{i}}{2}+\ln \left(1+e^{-\beta \omega_{i}}\right) .
\end{aligned}
$$

Now one can write

$$
\begin{gathered}
I_{1}=\frac{T}{-2 \pi i}(-2 \pi i)\left[\frac{\beta}{2}\left(\omega_{L}^{+}+\omega_{R}^{-}\right)+\ln \left(1+e^{-\beta \omega_{L}^{+}}\right)+\ln \left(1+e^{-\beta \omega_{R}^{-}}\right)\right] \\
=\frac{1}{2}\left(\omega_{L}^{+}+\omega_{R}^{-}\right)+T \ln \left(1+e^{-\beta \omega_{L}^{+}}\right)+T \ln \left(1+e^{-\beta \omega_{R}^{-}}\right), \\
I_{2}=\frac{1}{2}\left(\omega_{L}^{-}+\omega_{R}^{+}\right)+T \ln \left(1+e^{-\beta \omega_{L}^{-}}\right)+T \ln \left(1+e^{-\beta \omega_{R}^{+}}\right), \\
I_{3}=\frac{1}{2}\left(\omega_{R}^{+}+\omega_{L}^{-}\right)+T \ln \left(1+e^{-\beta \omega_{R}^{+}}\right)+T \ln \left(1+e^{-\beta \omega_{L}^{-}}\right), \\
I_{4}=\frac{1}{2}\left(\omega_{L}^{+}+\omega_{R}^{-}\right)+T \ln \left(1+e^{-\beta \omega_{L}^{+}}\right)+T \ln \left(1+e^{-\beta \omega_{R}^{-}}\right), \\
I_{0}=2 p+4 T \ln \left(1+e^{-\beta p}\right) .
\end{gathered}
$$

As found $I_{1}=I_{3}$ and $I_{2}=I_{4}$. 
Now one can write the quasiparticle part of the quark free energy as

$$
\begin{aligned}
F_{\mathrm{QP}}^{q}= & -N_{c} \sum_{f} \int \frac{d^{3} p}{(2 \pi)^{3}}\left[\left(\omega_{L}^{+}+\omega_{R}^{+}+\omega_{L}^{-}+\omega_{R}^{-}-2 p\right)+2 T \ln \left(1+e^{-\beta \omega_{L}^{+}}\right)\right. \\
& \left.+2 T \ln \left(1+e^{-\beta \omega_{R}^{+}}\right)+2 T \ln \frac{1+e^{-\beta \omega_{L}^{-}}}{1+e^{-\beta p}}+2 T \ln \frac{1+e^{-\beta \omega_{R}^{-}}}{1+e^{-\beta p}}\right]
\end{aligned}
$$

At large momentum limit, the QP modes are obtained as

$$
\begin{aligned}
& \omega_{L}^{+} \approx p+\frac{1}{p}\left(m_{\mathrm{th}}^{2}+m_{\mathrm{eff}}^{2} \frac{p_{z}}{p}\right)-\frac{1}{2 p^{3}}\left[\left(m_{\mathrm{th}}^{2}+m_{\mathrm{eff}}^{2} \frac{p_{z}}{p}\right)^{2}-\frac{m_{\mathrm{eff}}^{4} p_{\perp}^{2}}{p^{2}} \log \frac{2 p^{2}}{m_{\mathrm{th}}^{2}+m_{\mathrm{eff}}^{2} \frac{p_{z}}{p}}\right] \log \frac{2 p^{2}}{m_{\mathrm{th}}^{2}+m_{\mathrm{eff}}^{2} \frac{p_{z}}{p}}, \\
& \omega_{R}^{+} \approx p+\frac{1}{p}\left(m_{\mathrm{th}}^{2}-m_{\mathrm{eff}}^{2} \frac{p_{z}}{p}\right)-\frac{1}{2 p^{3}}\left[\left(m_{\mathrm{th}}^{2}-m_{\mathrm{eff}}^{2} \frac{p_{z}}{p}\right)^{2}-\frac{m_{\mathrm{eff}}^{4} p_{\perp}^{2}}{p^{2}} \log \frac{2 p^{2}}{m_{\mathrm{th}}^{2}-m_{\mathrm{eff}}^{2} \frac{p_{z}}{p}}\right] \log \frac{2 p^{2}}{m_{\mathrm{th}}^{2}-m_{\mathrm{eff}}^{2} \frac{p_{z}}{p}} .
\end{aligned}
$$

As can be seen, the terms in the parentheses in Eq. (D14) have divergences in the high momentum limit and one needs counterterms to regulate them. We regulate as below through the subtraction method following Ref. [107].

Now we construct the $\mathcal{F}_{q, \mathrm{QP}}^{\text {sub }}$ for $\omega_{L}^{+}$poles as

$$
\begin{aligned}
& \mathcal{F}_{q, Q P}^{\mathrm{sub}, L^{+}}=-N_{c} \sum_{f} \int_{p}\left[\sqrt{p^{2}+2 m_{\mathrm{th}}^{2}+\frac{2 m_{\mathrm{eff}}^{2} p_{z}}{p}}\right. \\
& -\frac{\left(m_{\mathrm{th}}^{2}+\frac{m_{\mathrm{eff}}^{2} p_{z}}{p}\right)^{2}}{2\left(p^{2}+2 m_{\mathrm{th}}^{2}+\frac{2 m_{\mathrm{eff}}^{2} p_{z}}{p}\right)^{3 / 2}}\left\{\log \frac{2\left(p^{2}+2 m_{\mathrm{th}}^{2}+\frac{2 m_{\mathrm{eff}}^{2} p_{z}}{p}\right)}{m_{\mathrm{th}}^{2}+\frac{m_{\mathrm{eff}}^{2} p_{z}}{p}}\right. \\
& \left.\left.\times\left(1+\frac{m_{\mathrm{eff}}^{4} p_{\perp}^{2}}{4 p^{4}\left\{m_{\mathrm{th}}^{2}+\frac{m_{\mathrm{eff}}^{2} p_{z}}{p}\right\}}-\frac{m_{\mathrm{eff}}^{4} p_{\perp}^{2}}{4 p^{2}\left\{m_{\mathrm{th}}^{2}+\frac{m_{\mathrm{eff}}^{2} p_{z}}{p}\right\}^{2}} \log \frac{2\left(p^{2}+2 m_{\mathrm{th}}^{2}+\frac{2 m_{\mathrm{eff}}^{2} p_{z}}{p}\right)}{m_{\mathrm{th}}^{2}+\frac{m_{\mathrm{eff}}^{2} p_{z}}{p}}\right)-1\right\}\right],
\end{aligned}
$$

and for the $\omega_{R}^{+}$poles as

$$
\begin{aligned}
\mathcal{F}_{q, Q P}^{\text {sub }, R^{+}}= & -N_{c} \sum_{f} \int_{p}\left[\sqrt{p^{2}+2 m_{\mathrm{th}}^{2}-\frac{2 m_{\mathrm{eff}}^{2} p_{z}}{p}}\right. \\
& -\frac{\left(m_{\mathrm{th}}^{2}-\frac{m_{\mathrm{eff}}^{2} p_{z}}{p}\right)^{2}}{2\left(p^{2}+2 m_{\mathrm{th}}^{2}-\frac{2 m_{\mathrm{eff}}^{2} p_{z}}{p}\right)^{3 / 2}}\left\{\log \frac{2\left(p^{2}+2 m_{\mathrm{th}}^{2}-\frac{2 m_{\mathrm{eff}}^{2} p_{z}}{p}\right)}{m_{\mathrm{th}}^{2}-\frac{m_{\mathrm{eff}}^{2} p_{z}}{p}}\right. \\
& \left.\left.\times\left(1+\frac{m_{\mathrm{eff}}^{4} p_{\perp}^{2}}{4 p^{4}\left\{m_{\mathrm{th}}^{2}-\frac{m_{\mathrm{eff}}^{2} p_{z}}{p}\right\}}-\frac{m_{\mathrm{eff}}^{4} p_{\perp}^{2}}{4 p^{2}\left\{m_{\mathrm{th}}^{2}-\frac{m_{\mathrm{eff}}^{2} p_{z}}{p}\right\}^{2}} \log \frac{2\left(p^{2}+2 m_{\mathrm{th}}^{2}-\frac{2 m_{\mathrm{eff}}^{2} p_{z}}{p}\right)}{m_{\mathrm{th}}^{2}-\frac{m_{\mathrm{eff}}^{2} p_{z}}{p}}\right)-1\right\}\right] .
\end{aligned}
$$

A regularized quasiparticle part is obtained by subtracting Eqs. (D15) and (D16) from (D14) as

$$
\begin{aligned}
F_{\mathrm{q}, \mathrm{QP}}^{\mathrm{ren}}= & -N_{c} \sum_{f} \int \frac{d^{3} p}{(2 \pi)^{3}}\left[2 T \ln \left(1+e^{-\beta \omega_{L}^{+}}\right)+2 T \ln \left(1+e^{-\beta \omega_{R}^{+}}\right)+2 T \ln \frac{1+e^{-\beta \omega_{L}^{-}}}{1+e^{-\beta p}}\right. \\
& \left.+2 T \ln \frac{1+e^{-\beta \omega_{R}^{-}}}{1+e^{-\beta p}}\right]-N_{c} \sum_{f} \int \frac{d^{3} p}{(2 \pi)^{3}}\left(\omega_{L}^{+}-\mathcal{F}_{q, q p}^{s u b, L^{+}}+\omega_{R}^{+}-\mathcal{F}_{q, q p}^{\mathrm{sub}, R^{+}}\right)-N_{c} \sum_{f} \int \frac{d^{3} p}{(2 \pi)^{3}}\left(\omega_{L}^{-}+\omega_{R}^{-}-2 p\right) .
\end{aligned}
$$


In the high momentum limit, $\omega_{L, R}^{-}$approaches to the light cone and $\left(\omega_{L}^{-}+\omega_{R}^{-}-2 p\right)$ vanishes. Also, the logarithmic terms that appear as a ratio in the above equation also vanish. The Stefan-Boltzmann limit of the above equation becomes

$$
F_{\mathrm{QP}}^{\mathrm{SB}}=-2 N_{c} N_{f} \int \frac{d^{3} p}{(2 \pi)^{3}}\left[p+2 T \ln \left(1+e^{-\beta p}\right)\right]
$$

Dropping the vacuum contribution, we get

$$
F_{\mathrm{QP}}^{\mathrm{SB}}=-N_{c} N_{f} \frac{7 \pi^{2} T^{4}}{180}
$$

Now, the Landau damping (LD) part $F_{\mathrm{LD}}^{q}$ of the free energy can be obtained as

$$
\begin{aligned}
F_{\mathrm{LD}}^{q} & =-\frac{2 N_{c}}{\pi} \sum_{f} \int \frac{d^{3} p}{(2 \pi)^{3}} \int_{0}^{p} d p_{0}\left(n_{F}\left(p_{0}\right)-\frac{1}{2}\right)\left[\operatorname{Arg}\left(L_{+}\right)+\operatorname{Arg}\left(R_{+}\right)+\operatorname{Arg}\left(L_{-}\right)+\operatorname{Arg}\left(R_{-}\right)\right]_{p_{0}<p} \\
& =\frac{2 N_{c}}{\pi} \sum_{f} \int \frac{d^{3} p}{(2 \pi)^{3}} \int_{0}^{p} d p_{0}\left(n_{F}\left(p_{0}\right)-\frac{1}{2}\right)\left[\Theta_{L D}^{L}+\Theta_{L D}^{R}\right],
\end{aligned}
$$

where

$$
\begin{aligned}
& \Theta_{L D}^{L}=\tan ^{-1} \frac{-\operatorname{Im}\left(L_{+} L_{-}\right)}{\operatorname{Re}\left(L_{+} L_{-}\right)}=\tan ^{-1} \frac{-\operatorname{Im}\left(L^{2}\right)}{\operatorname{Re}\left(L^{2}\right)}, \\
& \Theta_{L D}^{R}=\tan ^{-1} \frac{-\operatorname{Im}\left(R_{+} R_{-}\right)}{\operatorname{Re}\left(R_{+} R_{-}\right)}=\tan ^{-1} \frac{-\operatorname{Im}\left(R^{2}\right)}{\operatorname{Re}\left(R^{2}\right)} .
\end{aligned}
$$

Now we obtain the relevant contributions as

$$
\begin{gathered}
\operatorname{Im}\left(L^{2}\right)=\frac{\pi m_{\mathrm{th}}^{4}}{p^{2}}\left[\frac{p_{0}}{p}\left(1-\mu^{2} \xi^{2}\right)-\frac{\mu \xi\left(p^{2}-p_{0}^{2}\right)}{m_{\mathrm{th}}^{2}}+\frac{1}{2}\left\{\left(1-\frac{p_{0}^{2}}{p^{2}}\right)-\mu^{2}\left(1-\frac{\xi^{2} p_{0}^{2}}{p^{2}}\right)\right\} L_{g}\right], \\
\operatorname{Re}\left(L^{2}\right)=-\left[p^{2}-p_{0}^{2}+2 m_{\mathrm{th}}^{2}+\frac{2 m_{\mathrm{eff}}^{2} \xi p_{0}}{p}+\frac{m_{\mathrm{th}}^{4}}{p^{2}}\left\{\left(1-\mu^{2} \xi^{2}\right)-\left(\frac{p_{0}\left(1-\mu^{2} \xi^{2}\right)}{p}-\frac{\mu \xi\left(p^{2}-p_{0}^{2}\right)}{m_{\mathrm{th}}^{2}}\right) L_{g}\right.\right. \\
\left.\left.-\frac{\left(1-\mu^{2}\right) p^{2}-p_{0}^{2}\left(1-\mu^{2} \xi^{2}\right)}{4 p^{2}}\left(L_{g}^{2}-\pi^{2}\right)\right\}\right], \\
\operatorname{Im}\left(R^{2}\right)=\frac{\pi m_{\mathrm{th}}^{4}}{p^{2}}\left[\frac{p_{0}}{p}\left(1-\mu^{2} \xi^{2}\right)+\frac{\mu \xi\left(p^{2}-p_{0}^{2}\right)}{m_{\mathrm{th}}^{2}}+\frac{1}{2}\left\{\left(1-\frac{p_{0}^{2}}{p^{2}}\right)-\mu^{2}\left(1-\frac{\xi^{2} p_{0}^{2}}{p^{2}}\right)\right\} L_{g}\right], \\
\operatorname{Re}\left(R^{2}\right)=-\left[p^{2}-p_{0}^{2}+2 m_{\mathrm{th}}^{2}-\frac{2 m_{\mathrm{eff}}^{2} \xi p_{0}}{p}+\frac{m_{\mathrm{th}}^{4}}{p^{2}}\left\{\left(1-\mu^{2} \xi^{2}\right)-\left(\frac{p_{0}\left(1-\mu^{2} \xi^{2}\right)}{p}+\frac{\mu \xi\left(p^{2}-p_{0}^{2}\right)}{m_{\mathrm{th}}^{2}}\right) L_{g}\right.\right. \\
- \\
\left.\left.-\frac{\left(1-\mu^{2}\right) p^{2}-p_{0}^{2}\left(1-\mu^{2} \xi^{2}\right)}{4 p^{2}}\left(L_{g}^{2}-\pi^{2}\right)\right\}\right],
\end{gathered}
$$

with $\xi=\cos \theta ; \quad \mu=\frac{m_{\mathrm{eff}}^{2}}{m_{\mathrm{th}}^{2}}, \quad L_{g}=\log \frac{p+p_{0}}{p-p_{0}}$.

Now, because of the term $-\frac{1}{2}$ with the distribution function in Eq. (D20), the LD part will be UV diverging and we need a suitable subtraction term to regularize it. We choose the following subtraction terms [107] for $L$ and $R$ branches as 
$F_{\mathrm{LD}}^{\mathrm{sub}, \mathrm{L}}=-2 m_{\mathrm{th}}^{4} \int_{p} \int d p_{0}\left[\frac{p_{0}\left(1-\mu^{2} \xi^{2}-\frac{p}{p_{0}} \frac{\mu \xi\left(p^{2}-p_{0}^{2}\right)}{m_{\mathrm{th}}^{2}}\right)}{p^{3}\left(p^{2}-p_{0}^{2}+2 m_{\mathrm{th}}^{2}+\frac{2 m_{\mathrm{eff}}^{2} p_{0}^{\xi}}{p}\right)}+\frac{\left(1+\mu^{2} \xi^{2}\right)\left(p^{2}-p_{0}^{2}\right)-\mu^{2}\left(1-\xi^{2}\right) p^{2}}{2 p^{4}\left(p^{2}-p_{0}^{2}+2 m_{\mathrm{th}}^{2}+\frac{2 m_{\mathrm{eff}}^{2} p_{0} \xi}{p}\right)} \log \frac{p+p_{0}}{p-p_{0}}\right]$,

$F_{\mathrm{LD}}^{\mathrm{sub}, \mathrm{R}}=-2 m_{\mathrm{th}}^{4} \int_{p} \int d p_{0}\left[\frac{p_{0}\left(1-\mu^{2} \xi^{2}+\frac{p}{p_{0}} \frac{\mu \xi\left(p^{2}-p_{0}^{2}\right)}{m_{\mathrm{th}}^{2}}\right)}{p^{3}\left(p^{2}-p_{0}^{2}+2 m_{\mathrm{th}}^{2}-\frac{2 m_{\mathrm{eff}}^{2} p_{0} \xi}{p}\right)}+\frac{\left(1+\mu^{2} \xi^{2}\right)\left(p^{2}-p_{0}^{2}\right)-\mu^{2}\left(1-\xi^{2}\right) p^{2}}{2 p^{4}\left(p^{2}-p_{0}^{2}+2 m_{\mathrm{th}}^{2}-\frac{2 m_{\mathrm{eff}}^{2} p_{0} \xi}{p}\right)} \log \frac{p+p_{0}}{p-p_{0}}\right]$.

Total LD part of the quark free energy becomes

$$
F_{\mathrm{q}, \mathrm{LD}}^{\mathrm{ren}}=\frac{N_{c}}{2 \pi^{3}} \sum_{f} \int_{0}^{\infty} p^{2} d p \int_{0}^{p} d p_{0}\left[\frac{1}{e^{\beta p_{0}}+1}-\frac{1}{2}\right]\left[\theta_{\mathrm{LD}}^{L}+\theta_{\mathrm{LD}}^{R}\right]-F_{\mathrm{LD}}^{\mathrm{sub}, \mathrm{L}}-F_{\mathrm{LD}}^{\mathrm{sub}, \mathrm{R}}
$$

\section{Gluonic part}

Free energy of a gluon within the weak magnetic field approximation up to $\mathcal{O}(e B)^{2}$ is given by

$$
F_{g}=d_{A}\left(\mathcal{F}_{g}^{1}+\mathcal{F}_{g}^{2}+\mathcal{F}_{g}^{3}\right)
$$

where $\mathcal{F}_{g}^{1}, \mathcal{F}_{g}^{2}$, and $\mathcal{F}_{g}^{3}$ are defined in Eqs. (48a), (48b), and (48c). Similar to the quark case, we get the quasiparticle contribution of the $b$ mode of a gluon as

$$
\mathcal{F}_{g, Q P}^{1}=\int_{\mathbf{p}}\left[T \ln \left(1-e^{-\beta \omega_{b}}\right)+\frac{\omega_{b}}{2}\right] .
$$

We can also write the quasiparticle contribution of $c$ and $d$ modes as

$$
\begin{aligned}
& \mathcal{F}_{g, Q P}^{2}=\int_{\mathbf{p}}\left[T \ln \left(1-e^{-\beta \omega_{c}}\right)+\frac{\omega_{c}}{2}\right], \\
& \mathcal{F}_{g, Q P}^{3}=\int_{\mathbf{p}}\left[T \ln \left(1-e^{-\beta \omega_{d}}\right)+\frac{\omega_{d}}{2}\right] .
\end{aligned}
$$

So the total quasiparticle contribution of the gluon free energy is given by

$$
\mathcal{F}_{g, Q P}=d_{A} \int_{\mathbf{p}}\left[T \ln \frac{1-e^{-\beta \omega_{b}}}{1-e^{-\beta p}}+\frac{\omega_{b}-p}{2}+T \ln \left(1-e^{-\beta \omega_{c}}\right)+T \ln \left(1-e^{-\beta \omega_{d}}\right)+\frac{\omega_{c}+\omega_{d}}{2}\right] .
$$

In the above equation, all the logarithmic terms are a converging integral. $\frac{\left(\omega_{b}-p\right)}{2}$ is also UV converging as $\omega_{b}$ approaches to a light cone at large momentum. The last term is UV diverging and we need to regulate that with appropriate counterterms. Below, we write down the asymptotic form of $\omega_{c}$ at large momentum as 


$$
\begin{aligned}
\omega_{c}^{\text {asy }} \approx & p+\frac{m_{D}^{2}}{4 p}+\frac{m_{D}^{4}}{32 p^{3}}\left(3-2 \log \frac{8 p^{2}}{m_{D}^{2}}\right) \\
& +\sum_{f} \frac{q_{f} B^{2} g^{2}\left[\left(\pi m_{f}-4 T+32 m_{f}^{2} T g_{k}\right)\left(190 \cos 2 \theta_{p}+125 \cos 4 \theta_{p}-27\right)-8192 m_{f}^{2} T g_{k}\right]}{12288 \pi^{2} m_{f}^{2} p T} \\
& -\sum_{f} \frac{g^{2} q_{f} B^{2}\left(10 \cos 2 \theta_{p}+5 \cos 4 \theta_{p}-23\right)\left(32 m_{f}^{2} T g_{k}+\pi m_{f}-4 T\right) \log \frac{8 p^{2}}{m_{D}^{2}}}{2048 m_{f}^{2} p T \pi^{2}} \\
& +\sum_{f} \frac{m_{D}^{2} g^{2} q_{f} B^{2}\left[\left(32 m_{f}^{2} T g_{k}+\pi m_{f}-4 T\right)\left(970 \cos 2 \theta_{p}+1055 \cos 4 \theta_{p}-201\right)-16384 m_{f}^{2} T g_{k}\right]}{49152 \pi^{2} m_{f}^{2} p^{3} T} \\
& -\sum_{f} \frac{m_{D}^{2} g^{2} q_{f} B^{2}\left[\left(32 m_{f}^{2} T g_{k}+\pi m_{f}-4 T\right)\left(670 \cos 2 \theta_{p}+545 \cos 4 \theta_{p}-591\right)-8192 m_{f}^{2} T g_{k}\right] \log \frac{8 p^{2}}{m_{D}^{2}}}{49152 \pi^{2} m_{f}^{2} p^{3} T} \\
& +\sum_{f} \frac{m_{D}^{2} g^{2} q_{f} B^{2}\left(10 \cos 2 \theta_{p}+5 \cos 4 \theta_{p}-23\right)\left(32 m_{f}^{2} T g_{k}+\pi m_{f}-4 T\right) \log ^{2} \frac{8 p^{2}}{m_{D}^{2}}}{8192 \pi^{2} m_{f}^{2} p^{3} T} .
\end{aligned}
$$

Similarly, we can write down the asymptotic form of $\omega_{d}$ at large momentum as

$$
\begin{aligned}
\omega_{d}^{\text {asy }} \approx & p+\frac{m_{D}^{2}}{4 p}+\frac{m_{D}^{4}}{32 p^{3}}\left(3-2 \ln \frac{8 p^{2}}{m_{D}^{2}}\right)+\sum_{f} \frac{g^{2}\left(q_{f} B\right)^{2}}{6144 \pi^{2} m_{f}^{2} p T}\left[\frac{128 m_{f}\left(13-11 \cos 2 \theta_{p}\right) \cot ^{2} \theta_{p}}{3\left(\cosh \left(m_{f} / T\right)+1\right)}+27 \pi m_{f}\right. \\
& +60 T+\left(356 T-71 \pi m_{f}\right) \cos 2 \theta_{p}+8\left(16 T-7 \pi m_{f}\right) \csc ^{2} \theta_{p}+8 m_{f}^{2} T\left\{32\left(53-9 \cos 2 \theta_{p}\right) f_{k} \cot ^{2} \theta_{p}\right. \\
& \left.-\left(193+308 \cos 2 \theta_{p}-53 \cos 4 \theta_{p}\right) g_{k} \csc ^{2} \theta_{p}\right\}+12 \log \frac{8 p^{2}}{m_{D}^{2}}\left\{\frac{32 m_{f}\left(\cos 2 \theta_{p}-2\right) \cot ^{2} \theta_{p}}{3\left(\cosh \left(\frac{m_{f}}{T}\right)+1\right)}+46 T-8 \pi m_{f}\right. \\
& \left.\left.+\left(\pi m_{f}-6 T\right)\left(\cos 2 \theta_{p}+8 \csc ^{2} \theta_{p}\right)-16 m_{f}^{2} T\left(4\left(9-\cos 2 \theta_{p}\right) f_{k} \cot ^{2} \theta_{p}+g_{k}\left(9-\cos 2 \theta_{p}-8 \csc ^{2} \theta_{p}\right)\right)\right\}\right] \\
& -\sum_{f} \frac{m_{D}^{2} g^{2}\left(q_{f} B\right)^{2} \csc ^{2} \theta_{p}}{98305 \pi^{2} m_{f}^{2} p^{3} T}\left[\frac{512 m_{f}\left(64-77 \cos 2 \theta_{p}\right) \cos ^{2} \theta_{p}}{3\left(\cosh \left(m_{f} / T\right)+1\right)}-4 \cos 2 \theta_{p}\left(247 \pi m_{f}-796 T\right)-621 \pi m_{f}\right. \\
& +2172 T+\left(713 \pi m_{f}-3308 T\right) \cos 4 \theta_{p}-32 m_{f}^{2} T\left\{32 f_{k}\left(57 \cos 2 \theta_{p}-233\right) \cos ^{2} \theta_{p}+g_{k}\left(1436 \cos 2 \theta_{p}\right.\right. \\
& \left.\left.-599 \cos 4 \theta_{p}+955\right)\right\}+\left\{32 m_{f}^{2} T\left(128 f_{k}\left(9 \cos 2 \theta_{p}-53\right) \cos \theta_{p}+g_{k}\left(1124 \cos 2 \theta_{p}-257 \cos 4 \theta_{p}+733\right)\right)\right. \\
& -\frac{2048 m_{f}\left(13-11 \cos 2 \theta_{p}\right) \cos ^{2} \theta_{p}+20\left(77 \pi m_{f}-404 T\right) \cos 2 \theta_{p}+\left(1604 T-329 \pi m_{f}\right) \cos 4 \theta_{p}+1221 \pi m_{f}}{3\left(\cosh \left(m_{f} / T\right)+1\right)} \\
& -7092 T\} \log \frac{8 p^{2}}{m_{D}^{2}}+12\left\{\frac{128 m_{f} \cos \theta_{p}\left(2-\cos 2 \theta_{p}\right)}{3\left(\cosh \left(\frac{m_{f}}{T}\right)+1\right)}+2\left(52 T-9 \pi m_{f}\right) \cos 2 \theta_{p}+\left(\pi m_{f}-6 T\right) \cos 4 \theta_{p}\right. \\
& \left.\left.-15 \pi m_{f}+94 T-16 m_{f}^{2} T\left(16 f_{k}\left(\cos 2 \theta_{p}-9\right) \cos ^{2} \theta_{p}+g_{k}\left(20 \cos 2 \theta_{p}-\cos 4 \theta_{p}+13\right)\right)\right\} \log { }^{2} \frac{8 p^{2}}{m_{D}^{2}}\right]
\end{aligned}
$$

\section{a. Counterterm}

The appropriate counterterms should make the integral in Eq. (D34) ultraviolet convergent, and it should not introduce any infrared divergences. Our choice for the subtraction counterterm for $c$ mode is 


$$
\begin{aligned}
\omega_{c}^{C T}= & {\left[p^{2}+\frac{m_{D}^{2}}{2}+\sum_{f} \frac{q_{f} B^{2} g^{2}\left[\left(\pi m_{f}-4 T+32 m_{f}^{2} T g_{k}\right)\left(190 \cos 2 \theta_{p}+125 \cos 4 \theta_{p}-27\right)-8192 m_{f}^{2} T g_{k}\right]}{6144 \pi^{2} m_{f}^{2} T}\right.} \\
& \left.-\sum_{f} \frac{g^{2} q_{f} B^{2}\left(10 \cos 2 \theta_{p}+5 \cos 4 \theta_{p}-23\right)\left(32 m_{f}^{2} T g_{k}+\pi m_{f}-4 T\right)}{1024 m_{f}^{2} T \pi^{2}} \log \left\{\frac{8\left(p^{2}+m_{D}^{2} / 2\right)}{m_{D}^{2}}\right\}\right]^{1 / 2} \\
& -\frac{\left(m_{D}^{2}+\sum_{f} \frac{q_{f} B^{2} g^{2}\left[\left(\pi m_{f}-4 T+32 m_{f}^{2} T g_{k}\right)\left(160 \cos 2 \theta_{p}+155 \cos 4 \theta_{p}-63\right)-3072 m_{f}^{2} T g_{k}\right]}{1536 \pi^{2} m_{f}^{2}}\right)^{2}}{16\left(p^{2}+\frac{m_{D}^{2}}{2}\right)^{3 / 2}}\left[\left(1-\sum_{f} \frac{g^{2}\left(q_{f} B\right)^{2}}{3072 \pi^{2}}\right.\right. \\
& \times \frac{32 m_{f}^{2} T g_{k}\left(349-90 \cos 2 \theta_{p}+45 \cos 4 \theta_{p}\right)+9\left(\pi m_{f}-4 T\right)\left(53-10 \cos 2 \theta_{p}+5 \cos 4 \theta_{p}\right)}{m_{f}^{2} m_{D}^{2} T}-\sum_{f} \frac{g^{2}\left(q_{f} B\right)^{2}}{512} \\
& \left.\left.\times \frac{\left(10 \cos 2 \theta_{p}+5 \cos 4 \theta_{p}-23\right)\left(32 m_{f}^{2} T g_{k}+\pi m_{f}-4 T\right)}{\pi^{2} m_{f}^{2} m_{D}^{2} T} \log \frac{8 p^{2}+4 m_{D}^{2}}{m_{D}^{2}}\right) \log \frac{8 p^{2}+4 m_{D}^{2}}{m_{D}^{2}}-2\right],
\end{aligned}
$$

and for the $d$ mode is

$$
\begin{aligned}
& \omega_{d}^{C T}=\left[p^{2}+\frac{m_{D}^{2}}{2}+\sum_{f} \frac{g^{2}\left(q_{f} B\right)^{2}}{3072 \pi^{2} m_{f}^{2} T}\left\{\frac{128 m_{f}\left(13-11 \cos 2 \theta_{p}\right) \cot ^{2} \theta_{p}}{3\left(\cosh \left(m_{f} / T\right)+1\right)}+\left(356 T-71 \pi m_{f}\right) \cos 2 \theta_{p}+60 T+27 \pi m_{f}\right.\right. \\
& +8 m_{f}^{2} T \csc ^{2} \theta_{p}\left\{\frac{16}{m_{f}^{2}}-\frac{7 \pi}{m_{f} T}+32 f_{k}\left(53-9 \cos 2 \theta_{p}\right) \cos ^{2} \theta_{p}-g_{k}\left(308 \cos 2 \theta_{p}-53 \cos 4 \theta_{p}+193\right)\right\} \\
& +12\left(\frac{32 m_{f}\left(\cos 2 \theta_{p}-2\right) \cot ^{2} \theta_{p}}{3\left(\cosh \frac{m_{f}}{T}+1\right)}+\left(\pi m_{f}-6 T\right)\left(\cos 2 \theta_{p}+8 \csc ^{2} \theta_{p}\right)+46 T-8 \pi m_{f}\right. \\
& \left.\left.-16 m_{f}^{2} T\left\{4 f_{k}\left(9-\cos 2 \theta_{p}\right) \cot ^{2} \theta_{p}+g_{k}\left(9-\cos 2 \theta_{p}-8 \csc ^{2} \theta_{p}\right)\right\}\right) \log \frac{8 p^{2}+4 m_{D}^{2}}{m_{D}^{2}}\right\}^{1 / 2} \\
& -\frac{1}{16\left(p^{2}+\frac{m_{D}^{2}}{2}\right)^{3 / 2}}\left\{m_{D}^{2}-\sum_{f} \frac{g^{2}\left(q_{f} B\right)^{2} \csc ^{2} \theta_{p}}{9216 \pi^{2} m_{f}^{2} T}\left(\frac{64 m_{f} \cos ^{2} \theta_{p}\left(94 \cos 2 \theta_{p}-89\right)}{\cosh \left(m_{f} / T\right)+1}+405 \pi m_{f}-1764 T\right.\right. \\
& +6 \cos 2 \theta_{p}\left(101 \pi m_{f}-392 T\right)-3 \cos 4 \theta_{p}\left(101 \pi m_{f}-476 T\right)-1536 m_{f}^{2} T f_{k}\left(85-18 \cos 2 \theta_{p}\right) \cos ^{2} \theta_{p} \\
& \left.\left.+96 m_{f}^{2} T g_{k}\left(248 \cos 2 \theta_{p}-83 \cos 4 \theta_{p}+163\right)\right)\right\}^{2}\left[\left(1+\sum_{f} \frac{g^{2}\left(q_{f} B\right)^{2}}{512 \pi^{2} m_{b}^{2} m_{D}^{2} T}\left\{\frac{192 m_{f} \cot ^{2} \theta_{p}}{\cosh \left(\frac{m_{f}}{T}\right)+1}+179 m_{f} \pi\right.\right.\right. \\
& -1108 T+\left(21 \pi m_{f}-76 T\right) \cos 2 \theta_{p}+4\left(256 T-39 \pi m_{f}\right) \csc ^{2} \theta_{p}-256 m_{f}^{2} T f_{k}\left(\cos 2 \theta_{p}-23\right) \cot ^{2} \theta_{p} \\
& \left.-8 m_{f}^{2} T g_{k}\left(84 \cos 2 \theta_{p}+23 \cos 4 \theta_{p}+53\right) \csc ^{2} \theta_{p}\right\}+\sum_{f} \frac{g^{2}\left(q_{f} B\right)^{2}}{384 \pi^{2} m_{b}^{2} m_{D}^{2} T}\left\{\frac{32 m_{f}\left(\cos 2 \theta_{p}-2\right) \cot ^{2} \theta_{p}}{\cosh \left(m_{f} / T\right)+1}\right. \\
& +3\left(46 T-8 \pi m_{f}+\left(\pi m_{f}-6 T\right)\left(\cos 2 \theta_{p}+8 \csc ^{2} \theta_{p}\right)\right)-192 m_{f}^{2} T f_{k}\left(9-\cos 2 \theta_{p}\right) \cot ^{2} \theta_{p} \\
& \left.\left.\left.-48 m_{f}^{2} T g_{k}\left(9-\cos 2 \theta_{p}-8 \csc ^{2} \theta_{p}\right)\right\} \log \frac{8 p^{2}+4 m_{D}^{2}}{m_{D}^{2}}\right) \log \frac{8 p^{2}+4 m_{D}^{2}}{m_{D}^{2}}-2\right] \text {. }
\end{aligned}
$$

Using the counterterms in Eqs. (D37) and (D38), the total renormalized quasiparticle contribution of the gluon free energy from Eq. (D34) can be written as

$$
\begin{aligned}
F_{g, Q P}^{\mathrm{ren}}= & d_{A} \int \frac{p^{2} d p \sin \theta_{p} d \theta_{p} d \phi}{(2 \pi)^{3}}\left[T \ln \frac{1-e^{-\beta \omega_{b}}}{1-e^{-\beta p}}+\frac{\omega_{b}-p}{2}+T \ln \left(1-e^{-\beta \omega_{c}}\right)+T \ln \left(1-e^{-\beta \omega_{d}}\right)\right. \\
& \left.+\frac{\omega_{c}-\omega_{c}^{C T}}{2}+\frac{\omega_{d}-\omega_{d}^{C T}}{2}\right] .
\end{aligned}
$$




\section{Landau damping part}

\section{a. $b$ mode}

The Landau damping part for the $b$ mode in the gluon free energy is

$$
F_{g, L D}^{1}=\frac{1}{\pi} \int_{\mathbf{p}} \int_{0}^{p} d p_{0} \phi_{b}\left[\frac{1}{e^{\beta p_{0}}-1}+\frac{1}{2}\right]
$$

where the angle $\phi_{b}$ is given by

$$
\begin{aligned}
\phi_{b}= & \tan ^{-1} \frac{\operatorname{Im}\left(P^{2}-b\right)}{\operatorname{Re}\left(P^{2}-b\right)} \\
= & \tan ^{-1} \frac{\pi m_{D}^{2} p_{0}}{2 p^{3}+m_{D}^{2}\left(2 p-L p_{0}\right)}-\frac{2 \pi m_{D}^{2} \delta m_{D}^{2} p p_{0}}{\left\{2 p^{3}+m_{D}^{2}\left(2 p-L p_{0}\right)\right\}^{2}+\pi^{2} m_{D}^{4} p_{0}^{2}} \\
& -\sum_{f} \frac{p^{3} p_{0}\left(q_{f} B\right)^{2}}{768 \pi\left\{\left(2 p m_{D}^{2}+2 p^{3}-p_{0} L m_{D}^{2}\right)^{2}+\pi^{2} p_{0}^{2} m_{D}^{4}\right\}}\left[\frac{19 \pi}{m_{f} T}-\frac{9 \pi p_{0}^{2}}{m_{f} p^{2} T}\right. \\
& -\frac{8}{m_{f}^{2}}-\frac{384 p_{0}^{2}\left(f_{k}+g_{k}\right)}{p^{2}}+\frac{24 p_{0}^{2}}{m_{f}^{2} p^{2}}+\frac{m_{D}^{2}}{p^{2}}\left\{128\left(17 f_{k}+9 g_{k}\right)+\frac{19 \pi}{m_{f}^{2} T}-\frac{8 T}{m_{f}^{2} T}\right\} \\
& \left.+128\left(17 f_{k}+9 g_{k}\right)+3 \cos 2 \theta_{p}\left(128\left(f_{k}+g_{k}\right)-\frac{8}{m_{f}^{2}}+\frac{3 \pi}{m_{f} T}\right)\left(\frac{m_{D}^{2}}{p^{2}}-\frac{3 p_{0}^{2}}{p^{2}}+1\right)\right],
\end{aligned}
$$

where $L=\log \frac{p+p_{0}}{p-p_{0}}$. We must choose a subtraction term to remove the ultravilolet divergence in the $b$ mode without introducing any infrared divergences. Our choice for the subtraction term for the $b$ mode is

$$
\begin{aligned}
\mathcal{F}_{g, \mathrm{LD}}^{1, \text { sub }}= & \frac{1}{\pi} \int_{\mathbf{p}} \int_{0}^{p} \frac{d p_{0}}{2}\left[\frac{\pi m_{D}^{2} p_{0}}{2 p^{3}} \frac{2}{e^{\beta p_{0}}-1}+\frac{\pi m_{D}^{2} p_{0}}{2 p\left(p^{2}+m_{D}^{2}\right)}+\frac{\pi m_{D}^{4} p_{0}^{2} L}{4 p^{2}\left(p^{2}+m_{D}^{2}\right)^{2}}-\frac{\pi \delta m_{D}^{2} m_{D}^{2} p_{0}}{2 p\left(p^{2}+m_{D}^{2}\right)^{2}}\right. \\
& -\sum_{f} \frac{\left(q_{f} B\right)^{2} p_{0}}{768 m_{f}^{2} \pi T}\left\{\frac{5 \pi m_{f}+8 T+128 m_{f}^{2} T\left(7 f_{k}+3 g_{k}\right)-3 \cos 2 \theta_{p}\left(3 \pi m_{f}-8 T+128 m_{f}^{2} T\left(f_{k}+g_{k}\right)\right)}{p^{3}}\right\} \frac{1}{e^{\beta p_{0}}-1}-\sum_{f} \frac{g^{2}\left(q_{f} B\right)^{2} p_{0}}{1536 m_{f}^{2} \pi T} \\
& \left.+\frac{3 P^{2}\left\{3 \pi m_{f}-8 T+128 m_{f}^{2} T\left(f_{k}+g_{k}\right)\right\}\left(1+3 \cos 2 \theta_{p}\right)}{2 p^{5} T}\right\}-\sum_{f} \frac{g^{2} m_{D}^{2}\left(q_{f} B\right)^{2} p_{0}}{3072 \pi m_{f}^{2} T} \\
& \times\left\{\frac{128 m_{f}^{2} T\left(7 f_{k}+3 g_{k}\right)+5 \pi m_{f}+8 T-3 \cos \left(2 \theta_{p}\right)\left(128 m_{f}^{2} T\left(f_{k}+g_{k}\right)+3 \pi m_{f}-8 T\right)}{2 p\left(p^{2}+m_{D}^{2}\right)^{2}}\right. \\
& \left.-\frac{3 P^{2}\left(3 \cos 2 \theta_{p}+1\right)\left(128 m_{f}^{2} T\left(f_{k}+3 \pi m_{f}-8 T\right)\right.}{p\left(p^{2}+m_{D}^{2}\right)^{2}}\right\} \\
& \times\left\{\frac{8 T+29 \pi m_{f}+128 m_{f}^{2} T\left(31 f_{k}+15 g_{k}\right)-3 \cos 2 \theta_{p}\left(3 \pi m_{f}-8 T+128 m_{f}^{2} T\left(f_{k}+g_{k}\right)\right)}{\left(p^{2}+m_{D}^{2}\right)^{2}}\right. \\
& +\frac{4 p_{0}}{p} \frac{3 \cos 2 \theta_{p}\left(128 m_{f}^{2} T\left(f_{k}+g_{k}\right)+3 \pi m_{f}-8 T\right)-8 T\left(16 m_{f}^{2}\left(7 f_{k}+3 g_{k}\right)+1\right)-5 \pi m_{f}}{p\left(p^{2}+m_{D}^{2}\right)^{3}} \\
& \left.\left.\times\left(1-\frac{p_{0} L}{2 p}\right)-\frac{6 P^{2} p_{0}\left(1+3 \cos 2 \theta_{p}\right)\left(128 m_{f}^{2} T\left(f_{k}+g_{k}\right)+3 \pi m_{f}-8 T\right)}{2 p}\right\}\right] .
\end{aligned}
$$




\section{b. $c$ mode}

The Landau damping part for the $c$ mode in the gluon free energy is

$$
F_{g, L D}^{2}=-\frac{1}{\pi} \int_{\mathbf{p}} \int_{0}^{p} d \omega \phi_{c}\left[\frac{1}{e^{\beta \omega}-1}+\frac{1}{2}\right],
$$

where the angle $\phi_{c}$ is given by

$$
\begin{aligned}
& \phi_{c}=\tan ^{-1} \frac{\operatorname{Im}\left(P^{2}-c\right)}{\operatorname{Re}\left(P^{2}-c\right)} \\
& =\tan ^{-1} \frac{\pi m_{D}^{2} p_{0} P^{2}}{4 p^{3} P^{2}-2 m_{D}^{2} p p_{0}^{2}+m_{D}^{2} p_{0} P^{2} L}+\sum_{f} \frac{16\left(q_{f} B\right)^{2} g^{2} g_{k} m_{D}^{2} p^{3} p_{0} P^{2}}{3 \pi\left\{\left(2 m_{D}^{2} p p_{0}^{2}-4 p^{3} P^{2}-m_{D}^{2} p_{0} P^{2} L\right)^{2}+\pi^{2} m_{D}^{4} p_{0}^{2} P^{4}\right\}} \\
& +\sum_{f} \frac{g^{2} p_{0} p\left(q_{f} B\right)^{2}\left(32 g_{k} m_{f}^{2} T+\pi m_{f}-4 T\right)}{128 \pi m_{f}^{2} T\left\{\left(2 m_{D}^{2} p p_{0}^{2}-4 p^{3} P^{2}-m_{D}^{2} p_{0} P^{2} L\right)^{2}+\pi^{2} m_{D}^{4} p_{0}^{2} P^{4}\right\}}\left[\left\{\frac{m_{D}^{2} p_{0}}{p}\left(\frac{2 p_{0}}{p}-\frac{P^{2} L}{p^{2}}\right)-4 P^{2}\right\}\right. \\
& \times\left\{\frac{15}{4}\left(1-\cos \theta_{p} \cot \theta_{p}\right)\left(P^{4} \cos 2 \theta_{p}+8 P^{2} p_{0}^{2} \cos ^{2} \theta_{p}-\cos ^{4} \theta_{p}\left(p^{4}-10 p^{2} p_{0}^{2}+\frac{35 p_{0}^{4}}{3}\right)\right)+11 p^{4}-7 p^{2} p_{0}^{2}\right. \\
& \left.+5 p_{0}^{2} \cos 2 \theta_{p}\left(3 P^{2}+2 p_{0}^{2}\right)-10 p_{0}^{4} \csc ^{2} \theta_{p}+30 p_{0}^{4}\right\}-\frac{m_{D}^{2} P^{2}}{6 p^{2}}\left\{p^{2} p_{0}^{2}\left(97-40 \cos 2 \theta_{p}-25 \cos 4 \theta_{p}\right) \csc ^{2} \theta_{p}\right. \\
& -6 L p p_{0}\left(11 p^{2}+6 p_{0}^{2} \csc ^{2} \theta_{p}+5 p_{0}^{2}\right)+15 p^{4}\left(1-\cos \theta_{p} \cot \theta_{p}\right)\left(3 \sin ^{4} \theta_{p}-\frac{p_{0}^{2}}{p^{2}}\left(\frac{35 \cos ^{4} \theta_{p}}{3}-10 \cos ^{2} \theta_{p}+1\right)\right) \\
& +24\left\{\frac{15}{8} P^{4} \sin ^{4} \theta_{p}\left(\cos \theta_{p} \cot \theta_{p}-1\right)+3 P^{2} p_{0}^{2}\left(\csc ^{2} \theta_{p}+\frac{5}{4}\left(\sin ^{2} 2 \theta_{p}+2 \cos 2 \theta_{p}-2 \sin 2 \theta_{p} \cos ^{3} \theta_{p}\right)+2\right)\right. \\
& \left.\left.\left.+5 p_{0}^{4}\left(\frac{1}{5}-\cos ^{4} \theta_{p}+\cos 2 \theta_{p}-\frac{8 \cot ^{2} \theta_{p}}{5}+\cos ^{5} \theta_{p} \cot \theta_{p}\right)\right\}\left(1-\frac{L p_{0}}{2 p}\right)\right\}\right] \text {. }
\end{aligned}
$$

To remove the ultraviolet divergence without introducing any infrared divergences, our choice for the subtraction term for the $c$ mode is

$$
\begin{aligned}
\mathcal{F}_{g, \mathrm{LD}}^{2, \mathrm{sub}}= & -\frac{1}{\pi} \int_{\mathbf{p}} \int_{0}^{p} \frac{d p_{0}}{2}\left[\frac{\pi m_{D}^{2} p_{0}}{4 p^{3}} \frac{2}{e^{\beta p_{0}}-1}-\frac{\pi m_{D}^{2} p_{0} P^{2}}{4 p^{3}\left(-P^{2}+\frac{m_{D}^{2}}{2}\right)}-\frac{\pi m_{D}^{4} p_{0} P^{4}}{8 p^{5}\left(-P^{2}+\frac{m_{D}^{2}}{2}\right)^{2}}\left(\frac{p_{0}}{2 p} L-1\right)\right. \\
& +\sum_{f} \frac{5 g^{2}\left(q_{f} B\right)^{2}\left[32 g_{k} m_{f}^{2} T+\pi m_{f}-4 T\right]}{512 p^{3} \pi m_{f}^{2} T \sin ^{2} \theta_{p}} \frac{2}{e^{\beta p_{0}-1}}\left\{\frac{\frac{7}{5} \sin ^{2} \theta_{p}-\cos ^{4} \theta_{p}+\sin \theta_{p} \cos ^{6} \theta_{p}+\sin ^{4} \theta_{p} \cos ^{2} \theta_{p}}{p\left(p^{2}-p_{0}^{2}+\frac{m_{D}^{2}}{2}\right)}\right. \\
& +\frac{52+338 \cos 2 \theta_{p}+300 \cos 4 \theta_{p}-50 \cos 6 \theta_{p}-5 \sin \theta_{p}-45 \sin 3 \theta_{p}-65 \sin 5 \theta_{p}-25 \sin 7 \theta_{p}}{320 p^{3}} \\
& \left.+\frac{P^{2}\left(60+102 \cos 2 \theta_{p}+420 \cos 4 \theta_{p}-70 \cos 6 \theta_{p}+17 \sin \theta_{p}-3 \sin 3 \theta_{p}-55 \sin 5 \theta_{p}-35 \sin 7 \theta_{p}\right)}{512 p^{5}}\right\} \\
& +\sum_{f} \frac{5 g^{2}\left(q_{f} B\right)^{2} p_{0}\left[32 g_{k} m_{f}^{2} T+\pi m_{f}-4 T\right]}{256 \pi m_{f}^{2} T \sin ^{2} \theta_{p}}\left\{\frac{\frac{7}{5} \sin ^{2} \theta_{p}-\cos ^{4} \theta_{p}+\sin \theta_{p} \cos ^{6} \theta_{p}+\sin { }^{4} \theta_{p} \cos ^{2} \theta_{p}}{p\left(p^{2}-p_{0}^{2}+\frac{m_{D}^{2}}{2}\right)}\right. \\
& -\frac{52+338 \cos 2 \theta_{p}+300 \cos 4 \theta_{p}-50 \cos 6 \theta_{p}-5 \sin \theta_{p}-45 \sin 3 \theta_{p}-65 \sin 5 \theta_{p}-25 \sin 7 \theta_{p} P^{2}}{320 p^{3}\left(p^{2}-p_{0}^{2}+\frac{m_{D}^{2}}{2}\right)}
\end{aligned}
$$




$$
\begin{aligned}
& \left.+\frac{P^{6}\left(60+102 \cos 2 \theta_{p}+420 \cos 4 \theta_{p}-70 \cos 6 \theta_{p}+17 \sin \theta_{p}-3 \sin 3 \theta_{p}-55 \sin 5 \theta_{p}-35 \sin 7 \theta_{p}\right)}{512 p^{5}\left(p^{2}-p_{0}^{2}+\frac{m_{D}^{2}}{2}\right)^{2}}\right\} \\
& -\sum_{f} \frac{g^{2}\left(q_{f} B\right)^{2} m_{D}^{2} g_{k} p_{0} P^{2}}{3 \pi p^{3}\left(p^{2}-p_{0}^{2}+\frac{m_{D}^{2}}{2}\right)^{2}}+\sum_{f} \frac{5 g^{2}\left(q_{f} B\right)^{2} m_{D}^{2} p_{0}\left[32 g_{k} m_{f}^{2} T+\pi m_{f}-4 T\right] \csc ^{2} \theta_{p}}{32768 \pi m_{f}^{2} T} \\
& \times a\left\{-\frac{\frac{394}{5} \cos 2 \theta_{p}+12 \cos 4 \theta_{p}-2 \cos 6 \theta_{p}-5 \sin \theta_{p}-9 \sin 3 \theta_{p}-5 \sin 5 \theta_{p}-\sin 7 \theta_{p}-\frac{124}{5} \frac{P^{2} p_{0} L}{p}}{p^{3}\left(-P^{2}+\frac{m_{D}^{2}}{2}\right)^{2}}\right. \\
& -\frac{89 \sin \theta_{p}+213 \sin 3 \theta_{p}+161 \sin 5 \theta_{p}+37 \sin 7 \theta_{p}-\frac{8274}{5} \cos 2 \theta_{p}-444 \cos 4 \theta_{p}+74 \cos 6 \theta_{p}+\frac{1164}{5}}{12 p^{3}\left(-P^{2}+\frac{m_{D}^{2}}{2}\right)^{2}} P^{2} \\
& -\frac{41 \sin \theta_{p}+213 \sin 3 \theta_{p}+257 \sin 5 \theta_{p}+85 \sin 7 \theta_{p}-\frac{7602}{5} \cos 2 \theta_{p}-1020 \cos 4 \theta_{p}+170 \cos 6 \theta_{p}-\frac{948}{5}}{12 p^{5}\left(-P^{2}+\frac{m_{D}^{2}}{2}\right)^{2}} P^{4} \\
& +\frac{17 \sin \theta_{p}-3 \sin 3 \theta_{p}-55 \sin 5 \theta_{p}-35 \sin 7 \theta_{p}+102 \cos 2 \theta_{p}+420 \cos 4 \theta_{p}-70 \cos 6 \theta_{p}+60}{4 p^{7}\left(-P^{2}+\frac{m_{D}^{2}}{2}\right)^{3}} P^{8}\left[\frac{L p_{0}}{2 p}-1\right]
\end{aligned}
$$

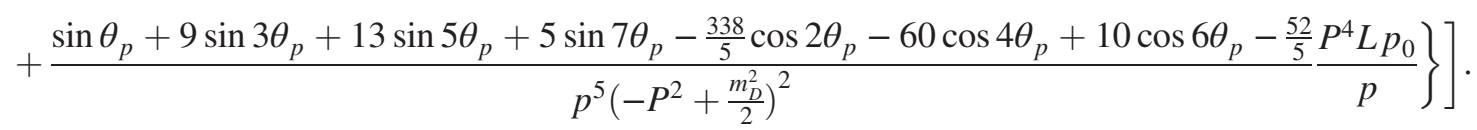

\section{c. $d$ mode}

The Landau damping part for the $c$ mode in the gluon free energy is

$$
F_{g, L D}^{3}=-\frac{1}{\pi} \int_{\mathbf{p}} \int_{0}^{p} d \omega \phi_{d}\left[\frac{1}{e^{\beta \omega}-1}+\frac{1}{2}\right]
$$

where the angle $\phi_{d}$ is given by

$$
\begin{aligned}
\phi_{d}= & \tan ^{-1} \frac{\operatorname{Im}\left(P^{2}-d\right)}{\operatorname{Re}\left(P^{2}-d\right)} \\
= & \tan ^{-1} \frac{\pi m_{D}^{2} p_{0} P^{2}}{4 p^{3} P^{2}-2 m_{D}^{2} p p_{0}^{2}+m_{D}^{2} p_{0} P^{2} L}-\sum_{f} \frac{g^{2} p_{0} p\left(q_{f} B\right)^{2} \csc ^{2} \theta_{p}}{768 \pi^{2} m_{f}^{2} P^{2} T\left\{\left(\frac{2 m_{D}^{2} p p_{0}^{2}}{P^{2}}-L m_{D}^{2} p_{0}-4 p^{3}\right)^{2}+\pi^{2} m_{D}^{4} p_{0}^{2}\right\}} \\
& \times\left[6 \pi\left(32 g_{k} m_{f}^{2} T+\pi m_{f}-4 T\right)\left(m_{D}^{2} p_{0}^{2}+p^{4}+3 p_{0}^{2}\left(2 p^{2}-5 p_{0}^{2}\right)\right)+\left(\frac{2 m_{D}^{2} p_{0}^{2}}{p^{2} P^{2}}-\frac{L m_{D}^{2} p_{0}}{p^{3}}-4\right) \cos ^{2} \theta_{p}\right. \\
& \times\left\{\frac{128 \pi m_{f} p_{0}^{2}\left(\cos ^{2} \theta_{p}\left(3 p^{2}-5 p_{0}^{2}\right)+3 p_{0}^{2}\right)}{\cosh ^{2}\left(\frac{m_{f}}{T}\right)+1}-3 \pi^{2} m_{f}\left(3 p^{4}-28 p^{2} p_{0}^{2}+69 p_{0}^{4}\right)+12 \pi T\left(3 p^{4}-10 p^{2} p_{0}^{2}+71 p_{0}^{4}\right)\right. \\
& \left.-32 m_{f}^{2} T p_{0}^{4}\left\{8 f_{k}\left(\frac{56 p^{3}}{p_{0}^{3}}-\frac{27 \pi p^{2}}{p_{0}^{2}}-3 \pi\right)+3 \pi g_{k}\left(\frac{3 p^{4}}{p_{0}^{4}}-\frac{46 p^{2}}{p_{0}^{2}}+67\right)\right\}-\frac{3 \pi p_{0}^{2} \cos ^{2} \theta_{p}\left\{4 T\left(9+\frac{151 P^{2}}{2}\right)\right.}{p^{2}}\right) \\
& \left.\left.-\pi m_{f}\left(1+\frac{139 P^{2}}{p^{2}}\right)-\frac{15 p^{2}}{p_{0}^{2}}\left(32 g_{k} m_{f}^{2} T+\pi m_{f}-4 T\right)+32 m_{f}^{2} T g_{k}\left(\frac{16 f_{k}}{g_{k}}\left(\frac{3 P^{2}}{p^{2}}+2\right)+7-\frac{127 P^{2}}{p^{2}}\right)\right\}\right\} \\
& -128 m_{f} \pi m_{D}^{2} p_{0}^{2} \cos ^{2} \theta_{p}\left\{\frac{8+\frac{6 P^{2}}{p^{2}}-\frac{3 L p_{0}^{3}}{p^{3}}+\cos ^{2} \theta_{p}\left(\frac{5 L p_{0}^{3}}{p^{3}}-\frac{3 L p_{0}}{p}-\frac{10 p_{0}^{2}}{p^{2}}+\frac{8}{3}\right)}{\cosh _{\frac{m_{f}}{T}}+1}+\frac{3 \pi}{64}\left(29-\frac{69 p_{0}^{2}}{p^{2}}\right)\right.
\end{aligned}
$$




$$
\begin{aligned}
& -\frac{T}{16 m_{f}}\left(87-\frac{213 p_{0}^{2}}{p^{2}}\right)+\frac{m_{f} T}{2}\left(224 f_{k}+71 g_{k}+\frac{24 f_{k} p_{0}^{2}}{p^{2}}-\frac{201 g_{k} p_{0}^{2}}{p^{2}}\right)+\frac{3 \pi L}{128}\left[\frac{69 p_{0}^{3}}{p^{3}}-\frac{28 p_{0}^{2}}{p^{2}}+\frac{3 p}{p_{0}}\right. \\
& \left.-\frac{4 T}{\pi m_{f}}\left(\frac{71 p_{0}^{3}}{p^{3}}-\frac{10 p_{0}}{p}+\frac{3 p}{p_{0}}\right)-\frac{p_{0} 32 g_{k} m_{f} T}{\pi p}\left(\frac{8 f_{k}}{g_{k}}\left(\frac{p_{0}^{2}}{p^{2}}+9\right)-\frac{3 p^{2}}{p_{0}^{2}}-\frac{67 p_{0}^{2}}{p^{2}}+46\right)\right]-\frac{\pi \cos ^{2} \theta_{p}}{128} \\
& \times\left[\frac{32 m_{f} p_{0}^{2} T}{\pi p^{2}}\left(14 f_{k}+\frac{275 g_{k} p^{2}}{p_{0}^{2}}-381 g_{k}\right)-\frac{4 T}{\pi m_{f}}\left(275-\frac{453 p_{0}^{2}}{p^{2}}\right)-\frac{417 p_{0}^{2}}{p^{2}}+275+\frac{3 L}{2}\left\{\frac{15 p}{p_{0}}-\frac{138 p_{0}}{p}\right.\right. \\
& \left.\left.\left.\left.+\frac{139 p_{0}^{3}}{p^{3}}+\frac{32 g_{k} m_{f} T}{\pi}\left(\frac{16 f_{k}}{g_{k}}\left(\frac{p}{p_{0}}-\frac{3 p_{0}^{3}}{p^{3}}\right)+\frac{127 p_{0}^{3}}{p^{3}}-\frac{134 p_{0}}{p}+\frac{15 p}{p_{0}}\right)-\frac{4 T\left(\frac{151 p_{0}^{3}}{p^{3}}-\frac{142 p_{0}}{p}+\frac{15 p}{p_{0}}\right)}{\pi m_{f}}\right\}\right]\right\}\right] .
\end{aligned}
$$

Our choice for the subtraction term for the $d$ mode to remove the divergence is

$$
\begin{aligned}
& \mathcal{F}_{g, \mathrm{LD}}^{3, \text { sub }}=-\frac{1}{\pi} \int_{\mathbf{p}} \int_{0}^{p} \frac{d p_{0}}{2}\left[\frac{\pi m_{D}^{2} p_{0}}{4 p^{3}} \frac{2}{e^{\beta p_{0}}-1}-\frac{\pi m_{D}^{2} p_{0} P^{2}}{4 p^{3}\left(-P^{2}+\frac{m_{D}^{2}}{2}\right)}-\frac{\pi m_{D}^{4} p_{0} P^{4}}{8 p^{5}\left(-P^{2}+\frac{m_{D}^{2}}{2}\right)^{2}}\left(\frac{p_{0}}{2 p} L-1\right)\right. \\
& +\sum_{f} \frac{2 g^{2}\left(q_{f} B\right)^{2}}{e^{\beta p_{0}}-1}\left\{\frac{14 p_{0}^{2} f_{k} \cot ^{2} \theta_{p}}{3 \pi^{2} p^{2}\left(p^{2}-p_{0}^{2}+\frac{m_{D}^{2}}{2}\right)}-\frac{3 p_{0} \csc ^{2} \theta_{p}}{768 \pi m_{f}^{2} p T\left(p^{2}-p_{0}^{2}+\frac{m_{D}^{2}}{2}\right)}\left[\frac{32 m_{f}\left(2-\cos 2 \theta_{p}\right) \cos ^{2} \theta_{p}}{\cosh \frac{m_{f}}{T}+1}\right.\right. \\
& +\cos ^{2} \theta_{p}\left(640 m_{f}^{2} T f_{k}-192 m_{f}^{2} T g_{k}-11 \pi m_{f}+64 T\right)+2 \cos ^{4} \theta_{p}\left(16 m_{f}^{2} T\left(g_{k}-4 f_{k}\right)+\pi m_{f}-6 T\right) \\
& \left.+32 m_{f}^{2} T g_{k}+\pi m_{f}-4 T\right]+\frac{3 p_{0} \csc ^{2} \theta_{p}}{1536 \pi m_{f}^{2} p^{3} T}\left(\frac{32 m_{f}\left(5-7 \cos 2 \theta_{p}\right) \cos ^{2} \theta_{p}}{3\left(\cosh \left(\frac{m_{f}}{T}\right)+1\right)}+6\left(32 m_{f}^{2} T g_{k}+\pi m_{f}-4 T\right)\right. \\
& \left.+5 \cos ^{4} \theta_{p}\left(7 \pi m_{f}-32 T-64 m_{f}^{2} T\left(2 f_{k}-3 g_{k}\right)\right)+11 \cos ^{2} \theta_{p}\left(24 T-5 \pi m_{f}+128 m_{f}^{2} T\left(f_{k}-g_{k}\right)\right)\right) \\
& +\frac{3 p_{0} \csc ^{2} \theta_{p} P^{2}}{6144 \pi m_{f}^{2} p^{5} T}\left(\frac{128 m_{f}\left(1-5 \cos 2 \theta_{p}\right) \cos ^{2} \theta_{p}}{3\left(\cosh \frac{m_{f}}{T}+1\right)}+\cos ^{2} \theta_{p}\left(64 m_{f}^{2} T\left(8 f_{k}-67 g_{k}\right)-138 \pi m_{f}+568 T\right)\right. \\
& \left.\left.+15\left(32 m_{f}^{2} T g_{k}+\pi m_{f}-4 T\right)+\cos ^{4} \theta_{p}\left(139 \pi m_{f}-604 T-32 m_{f}^{2} T\left(48 f_{k}-127 g_{k}\right)\right)\right)\right\} \\
& +\sum_{f} g^{2}\left(q_{f} B\right)^{2}\left\{\frac{14 p_{0}^{2} f_{k} \cot ^{2} \theta_{p}}{3 \pi^{2} p^{2}\left(-P^{2}+\frac{m_{D}^{2}}{2}\right)}-\frac{3 p_{0} \csc ^{2} \theta_{p}}{768 \pi m_{f}^{2} p T\left(p^{2}-p_{0}^{2}+\frac{m_{D}^{2}}{2}\right)}\left[\frac{32 m_{f}\left(2-\cos 2 \theta_{p}\right) \cot ^{2} \theta_{p}}{\cosh \frac{m_{f}}{T}+1}\right.\right. \\
& +\cos ^{2} \theta_{p}\left(640 m_{f}^{2} T f_{k}-192 m_{f}^{2} T g_{k}-11 \pi m_{f}+64 T\right)+2 \cos ^{4} \theta_{p}\left(16 m_{f}^{2} T\left(g_{k}-4 f_{k}\right)+\pi m_{f}-6 T\right) \\
& \left.+32 m_{f}^{2} T g_{k}+\pi m_{f}-4 T\right]+\frac{3 p_{0} P^{2} \csc ^{2} \theta_{p}}{1536 \pi m_{f}^{2} p^{3} T\left(P^{2}-\frac{m_{D}^{2}}{2}\right)}\left[\frac{32 m_{f}\left(5-7 \cos 2 \theta_{p}\right) \cos ^{2} \theta_{p}}{3\left(\cosh \left(\frac{m_{f}}{T}\right)+1\right)}+6\left(32 m_{f}^{2} T g_{k}+\pi m_{f}\right)\right. \\
& \left.-24 T+5 \cos ^{4} \theta_{p}\left(7 \pi m_{f}-32 T-64 m_{f}^{2} T\left(2 f_{k}-3 g_{k}\right)\right)+11 \cos ^{2} \theta_{p}\left(24 T-5 \pi m_{f}+128 m_{f}^{2} T\left(f_{k}-g_{k}\right)\right)\right] \\
& +\frac{3 p_{0} \csc ^{2} \theta_{p} P^{4}}{6144 \pi m_{f}^{2} p^{5}\left(P^{2}-\frac{m_{D}^{2}}{2}\right) T}\left(\frac{128 m_{f}\left(1-5 \cos 2 \theta_{p}\right) \cos ^{2} \theta_{p}}{3\left(\cosh \frac{m_{f}}{T}+1\right)}+\cos ^{2} \theta_{p}\left(64 m_{f}^{2} T\left(8 f_{k}-67 g_{k}\right)-138 \pi m_{f}\right)\right. \\
& \left.\left.-568 T \cos ^{2} \theta_{p}+15\left(32 m_{f}^{2} T g_{k}+\pi m_{f}-4 T\right)+\cos ^{4} \theta_{p}\left(139 \pi m_{f}-604 T-32 m_{f}^{2} T\left(48 f_{k}-127 g_{k}\right)\right)\right)\right\} \\
& +\sum_{f} g^{2} m_{D}^{2}\left(q_{f} B\right)^{2}\left[\frac{\left(2-\cos 2 \theta_{p}\right) \cot ^{2} \theta_{p} P^{2} L}{48 \pi m_{f} p^{2} T\left(\cosh \frac{m_{f}}{T}+1\right)\left(P^{2}-\frac{m_{D}^{2}}{2}\right)^{2}}+\frac{7 P^{2} \cot ^{2} \theta_{p}}{3 \pi^{2} p^{2}\left(P^{2}-\frac{m_{D}^{2}}{2}\right)^{2}}\left\{f_{k}+\frac{3 \pi L}{1792}\left(\frac{29}{m_{f}^{2}}\right.\right.\right. \\
& \left.\left.-\frac{5 \pi}{m_{f} T}+288 f_{k}-88 g_{k}-\left(\frac{3}{m_{f}^{2}}-\frac{\pi}{2 m_{f} T}-8\left(g_{k}-4 f_{k}\right)\right) \cos 2 \theta_{p}+\left(16 g_{k}-\frac{2}{m_{f}^{2}}+\frac{\pi}{2 m_{f} T}\right) \sec ^{2} \theta_{p}\right)\right\}
\end{aligned}
$$




$$
\begin{aligned}
& -\frac{p_{0} P^{2}}{384 \pi p^{3}\left(P^{2}-\frac{m_{D}^{2}}{2}\right)^{2}}\left\{\frac{4\left(25-17 \cos 2 \theta_{p}\right) \cot ^{2} \theta_{p}}{3 m_{f} T\left(\cosh \frac{m_{f}}{T}+1\right)}+\frac{219 \pi}{32 m_{f} T}-\frac{261}{8 m_{f}^{2}}-\frac{448 f_{k} L}{\pi}-616 f_{k}+209 g_{k}\right. \\
& \left.+\frac{5 \cos 2 \theta_{p}}{T}\left(24 f_{k}-13 g_{k}+\frac{25}{8 m_{f}^{2}}-\frac{19 \pi}{32 m_{f}}\right)-16 \csc ^{2} \theta_{p}\left(\frac{31 \pi^{2}}{64 \pi m_{f} T}-\frac{5}{2 m_{f}^{2}}-\frac{28 f_{k} L}{\pi}-46 f_{k}+13 g_{k}\right)\right\} \\
& +\frac{P^{2} L}{16 \pi p^{4}\left(P^{2}-\frac{m_{D}^{2}}{2}\right)}\left\{\frac{112 f_{k} \cot ^{2} \theta_{p}}{3 \pi L}+\frac{3 \cos ^{2} \theta_{p}}{m_{f} T\left(\cosh \frac{m_{f}}{T}+1\right)}-\left(8 g_{k}-7 f_{k}-\frac{23}{16 m_{f}^{2}}+\frac{39 \pi}{128 m_{f} T}\right) \cos 2 \theta_{p}\right. \\
& \left.-21 f_{k}+4 g_{k}-\frac{29}{16 m_{f}^{2}}+\frac{37 \pi}{128 m_{f} T}-4 \csc ^{2} \theta_{p}\left(2 g_{k}-7 f_{k}-\frac{11}{16 m_{f}^{2}}+\frac{15 \pi}{128 m_{f} T}\right)\right\}-\frac{7 p_{0} P^{4}}{768 \pi p^{5}\left(P^{2}-\frac{m_{D}^{2}}{2}\right)^{2}} \\
& \times\left\{\frac{8\left(31-47 \cos \left(2 \theta_{p}\right) \cot ^{2} \theta_{p}\right.}{21 m_{f} T\left(\cosh \frac{m_{f}}{T}+1\right)}-\frac{309 \pi}{224 m_{f} T}+\frac{393}{56 m_{f}^{2}}+\frac{11 \cos 2 \theta_{p}}{7}\left(48 f_{k}-77 g_{k}+\frac{101}{8 m_{f}^{2}}-\frac{89 \pi}{32 m_{f} T}\right)\right. \\
& \left.-\frac{128 f_{k} L}{\pi}-80 f_{k}-23 g_{k}+\frac{16}{7} \csc ^{2} \theta_{p}\left(\frac{56 f_{k} L}{\pi}+68 f_{k}-23 g_{k}+\frac{25}{8 m_{f}^{2}}-\frac{11 \pi}{16 m_{f} T}\right)\right\}+\frac{P^{6} L}{256 \pi p^{6}\left(P^{2}-\frac{m_{D}^{2}}{2}\right)^{2}} \\
& \times\left\{\frac{8\left(5-7 \cos 2 \theta_{p}\right) \cot ^{2} \theta_{p}}{3 m_{f} T\left(\cosh \frac{m_{f}}{T}+1\right)}-\frac{121}{32}\left(32 g_{k}-\frac{4}{m_{f}^{2}}+\frac{\pi}{m_{f} T}\right)-\cos 2 \theta_{p}\left(247 g_{k}-128 f_{k}-\frac{311}{8 m_{f}^{2}}+\frac{279 \pi}{32 m_{f} T}\right)\right\} \\
& +\frac{p_{0}\left(1-5 \cos 2 \theta_{p}\right) \cot ^{2} \theta_{p} P^{8}\left(1-\frac{p_{0} L}{2 p}\right)}{48 \pi m_{f} p^{7} T\left(\cosh \frac{m_{f}}{T}+1\right)\left(P^{2}-\frac{m_{D}^{2}}{2}\right)^{3}}-\frac{p_{0} \csc ^{2} \theta_{p} P^{8}}{64 \pi p^{7}\left(P^{2}-\frac{m_{D}^{2}}{2}\right)^{3}}\left\{2 \cos ^{2} \theta_{p}\left(8 f_{k}-67 g_{k}+\frac{71}{8 m_{f}^{2}}-\frac{69 \pi}{32 m_{f} T}\right)\right. \\
& \left.+15 g_{k}-\frac{15}{8 m_{f}^{2}}+\frac{15 \pi}{32 m_{f} T}+\cos ^{4} \theta_{p}\left(127 g_{k}-48 f_{k}-\frac{151}{8 m_{f}^{2}}+\frac{139 \pi}{32 m_{f} T}\right)\right\} \\
& -\frac{P^{10} \csc ^{2} \theta_{p} L}{128 \pi p^{8}\left(P^{2}-\frac{m_{D}^{2}}{2}\right)^{3}}\left\{\frac{15}{8 m_{F}^{2}}-15 g_{k}-\frac{15 \pi}{32 m_{f} T}-\left(16 f_{k}-134 g_{k}+\frac{71}{4 m_{f}^{2}}-\frac{69 \pi}{16 m_{f} T}\right) \cos ^{2} \theta_{p}\right. \\
& \left.\left.+\cos ^{4} \theta_{p}\left(48 f_{k}-127 g_{k}+\frac{151}{8 m_{f}^{2}}-\frac{139 \pi}{32 m_{f} T}\right)\right\}\right] \text {. }
\end{aligned}
$$

Using the subtraction terms for $b, c$, and $d$ modes as given in Eqs. (D42), (D45), and (D48) respectively, the total renormalized LD contribution of the gluon free energy is given by

$$
\mathcal{F}_{g, \mathrm{Ld}}^{\mathrm{ren}}=d_{A}\left(\mathcal{F}_{g, \mathrm{Ld}}^{1}-\mathcal{F}_{g, \mathrm{Ld}}^{1, \mathrm{sub}}+\mathcal{F}_{g, \mathrm{Ld}}^{2}-\mathcal{F}_{g, \mathrm{Ld}}^{2, \mathrm{sub}}+\mathcal{F}_{g, \mathrm{Ld}}^{3}-\mathcal{F}_{g, \mathrm{Ld}}^{3, \mathrm{sub}}\right) .
$$

Therefore, the total renormalized pressure in terms of quasiparticle poles and the Landau-damping term come from Eqs. (D17), (D29), (D39), (D49) and become

$$
P^{Q P+L D}=-\left[F_{\mathrm{q}, \mathrm{QP}}^{\mathrm{ren}}+F_{\mathrm{q}, \mathrm{LD}}^{\mathrm{ren}}+F_{\mathrm{g}, \mathrm{QP}}^{\mathrm{ren}}+F_{\mathrm{g}, \mathrm{LD}}^{\mathrm{ren}}\right] .
$$

[1] I. A. Shovkovy, Magnetic catalysis: A review, Lect. Notes Phys. 871, 13 (2013).

[2] M. D'Elia, Lattice QCD simulations in external background fields, Lect. Notes Phys. 871, 181 (2013).

[3] K. Fukushima, Views of the chiral magnetic effect, Lect. Notes Phys. 871, 241 (2013).
[4] N. Mueller, J. A. Bonnet, and C. S. Fischer, Dynamical quark mass generation in a strong external magnetic field, Phys. Rev. D 89, 094023 (2014).

[5] V. A. Miransky and I. A. Shovkovy, Quantum field theory in a magnetic field: From quantum chromodynamics to graphene and Dirac semimetals, Phys. Rep. 576, 1 (2015). 
[6] A. Adare et al. (PHENIX Collaboration), Observation of Direct-Photon Collective Flow in $\sqrt{s_{N N}}=200 \mathrm{GeV}$ $\mathrm{Au}+\mathrm{Au}$ Collisions, Phys. Rev. Lett. 109, 122302 (2012).

[7] G. Basar, D. Kharzeev, D. Kharzeev, and V. Skokov, Conformal Anomaly as a Source of Soft Photons in Heavy Ion Collisions, Phys. Rev. Lett. 109, 202303 (2012).

[8] A. Bzdak and V. Skokov, Anisotropy of Photon Production: Initial Eccentricity or Magnetic Field, Phys. Rev. Lett. 110, 192301 (2013).

[9] L. McLerran and V. Skokov, Comments about the electromagnetic field in heavy-ion collisions, Nucl. Phys. A929, 184 (2014).

[10] V. Skokov, A. Y. Illarionov, and V. Toneev, Estimate of the magnetic field strength in heavy-ion collisions, Int. J. Mod. Phys. A 24, 5925 (2009).

[11] R. C. Duncan and C. Thompson, Formation of very strongly magnetized neutron stars: Implications for gamma-ray bursts, Astrophys. J. 392, L9 (1992).

[12] S. Chakrabarty, D. Bandyopadhyay, and S. Pal, Dense Nuclear Matter in a Strong Magnetic Field, Phys. Rev. Lett. 78, 2898 (1997).

[13] D. Bandyopadhyay, S. Chakrabarty, and S. Pal, Quantizing Magnetic Field and Quark: Hadron Phase Transition in a Neutron Star, Phys. Rev. Lett. 79, 2176 (1997).

[14] D. E. Kharzeev, L. D. McLerran, and H. J. Warringa, The Effects of topological charge change in heavy ion collisions: Event by event $\mathrm{P}$ and $C P$ violation, Nucl. Phys. A803, 227 (2008).

[15] K. Fukushima, D. E. Kharzeev, and H. J. Warringa, The chiral magnetic effect, Phys. Rev. D 78, 074033 (2008).

[16] D. E. Kharzeev, Topologically induced local $\mathrm{P}$ and $C P$ violation in QCD x QED, Ann. Phys. (Amsterdam) 325, 205 (2010).

[17] J. Alexandre, K. Farakos, and G. Koutsoumbas, Magnetic catalysis in $\mathrm{QED}(3)$ at finite temperature: Beyond the constant mass approximation, Phys. Rev. D 63, 065015 (2001).

[18] V. P. Gusynin and I. A. Shovkovy, Chiral symmetry breaking in QED in a magnetic field at finite temperature, Phys. Rev. D 56, 5251 (1997).

[19] D. S. Lee, C. N. Leung, and Y. J. Ng, Chiral symmetry breaking in a uniform external magnetic field, Phys. Rev. D 55, 6504 (1997).

[20] G. S. Bali, F. Bruckmann, G. Endrodi, Z. Fodor, S. D. Katz, S. Krieg, A. Schafer, and K. K. Szabo, The QCD phase diagram for external magnetic fields, J. High Energy Phys. 02 (2012) 044.

[21] V. G. Bornyakov, P. V. Buividovich, N. Cundy, O. A. Kochetkov, and A. Schäfer, Deconfinement transition in two-flavor lattice QCD with dynamical overlap fermions in an external magnetic field, Phys. Rev. D 90, 034501 (2014).

[22] N. Mueller and J. M. Pawlowski, Magnetic catalysis and inverse magnetic catalysis in QCD, Phys. Rev. D 91, 116010 (2015).

[23] A. Ayala, M. Loewe, A. Z. Mizher, and Zamora, R., Inverse magnetic catalysis for the chiral transition induced by thermomagnetic effects on the coupling constant, Phys. Rev. D 90, 036001 (2014).

[24] R. L. S. Farias, K. P. Gomes, G. I. Krein, and M. B. Pinto, Importance of asymptotic freedom for the pseudocritical temperature in magnetized quark matter, Phys. Rev. C 90, 025203 (2014).

[25] A. Ayala, M. Loewe, and R. Zamora, Inverse magnetic catalysis in the linear sigma model with quarks, Phys. Rev. D 91, 016002 (2015).

[26] A. Ayala, M. Loewe, and R. Zamora, Inverse magnetic catalysis in the linear sigma model, J. Phys. Conf. Ser. 720, 012026 (2016).

[27] A. Ayala, C. A. Dominguez, L. A. Hernandez, M. Loewe, and R. Zamora, Inverse magnetic catalysis from the properties of the QCD coupling in a magnetic field, Phys. Lett. B 759, 99 (2016).

[28] S. S. Avancini, R. L. S. Farias, M. B. Pinto, W. R. Tavares, and V.S. Timóteo, $\pi_{0}$ pole mass calculation in a strong magnetic field and lattice constraints, Phys. Lett. B 767, 247 (2017).

[29] S. Fayazbakhsh and N. Sadooghi, Phase diagram of hot magnetized two-flavor color superconducting quark matter, Phys. Rev. D 83, 025026 (2011).

[30] S. Fayazbakhsh and N. Sadooghi, Color neutral 2SC phase of cold and dense quark matter in the presence of constant magnetic fields, Phys. Rev. D 82, 045010 (2010).

[31] J. O. Andersen, W. R. Naylor, and A. Tranberg, Phase diagram of QCD in a magnetic field: A review, Rev. Mod. Phys. 88, 025001 (2016).

[32] J. O. Andersen, Chiral perturbation theory in a magnetic background: Finite-temperature effects, J. High Energy Phys. 10 (2012) 005.

[33] M. Strickland, V. Dexheimer, and D. P. Menezes, Bulk properties of a fermi gas in a magnetic field, Phys. Rev. D 86, 125032 (2012).

[34] R. L. S. Farias, V. S. Timoteo, S. S. Avancini, M. B. Pinto, and G. Krein, Thermo-magnetic effects in quark matter: Nambu-Jona-Lasinio model constrained by lattice QCD, Eur. Phys. J. A 53, 101 (2017).

[35] S. Rath and B. K. Patra, One-loop QCD thermodynamics in a strong homogeneous and static magnetic field, J. High Energy Phys. 12 (2017) 098.

[36] S. Fayazbakhsh, S. Sadeghian, and N. Sadooghi, Properties of neutral mesons in a hot and magnetized quark matter, Phys. Rev. D 86, 085042 (2012).

[37] S. Fayazbakhsh and N. Sadooghi, Weak decay constant of neutral pions in a hot and magnetized quark matter, Phys. Rev. D 88, 065030 (2013).

[38] A. Bandyopadhyay and S. Mallik, Rho meson decay in the presence of a magnetic field, Eur. Phys. J. C 77, 771 (2017).

[39] A. Bandyopadhyay, R. L. S. Farias, and R. O. Ramos, Effect of the magnetized medium on the decay of neutral scalar bosons, Phys. Rev. D 98, 076007 (2018).

[40] P. Chakraborty, Meson spectral function and screening masses in magnetized quark gluon plasma, arXiv:1711 .04404 .

[41] R. Rougemont, R. Critelli, and J. Noronha, Holographic calculation of the QCD crossover temperature in a magnetic field, Phys. Rev. D 93, 045013 (2016).

[42] S. I. Finazzo, R. Critelli, R. Rougemont, and J. Noronha, Momentum transport in strongly coupled anisotropic plasmas in the presence of strong magnetic fields, Phys. Rev. D 94, 054020 (2016). 
[43] A. Ayala, J. D. Castano-Yepes, C. A. Dominguez, and L. A. Hernandez, Thermal photons from gluon fusion with magnetic fields, EPJ Web Conf. 141, 02007 (2017).

[44] N. Sadooghi and F. Taghinavaz, Magnetized plasminos in cold and hot QED plasmas, Phys. Rev. D 92, 025006 (2015).

[45] B. Karmakar, A. Bandyopadhyay, N. Haque, and M. G. Mustafa, General structure of gauge boson propagator and its spectra in a hot magnetized medium, Eur. Phys. J. C 79, 658 (2019)

[46] A. Ayala, C. A. Dominguez, S. Hernandez-Ortiz, L. A. Hernandez, M. Loewe, D. M. Paret, and R. Zamora, Gluon polarization tensor in a thermo-magnetic medium, arXiv: 1805.07344.

[47] A. Das, A. Bandyopadhyay, P. K. Roy, and M. G. Mustafa, General structure of fermion two-point function and its spectral representation in a hot magnetized medium, Phys. Rev. D 97, 034024 (2018).

[48] K. Hattori and D. Satow, Gluon spectrum in a quark-gluon plasma under strong magnetic fields, Phys. Rev. D 97, 014023 (2018).

[49] M. Kurian, S. Mitra, and V. Chandra, Transport coefficients of hot magnetized QCD matter, Eur. Phys. J. C 79, 134 (2019).

[50] M. Kurian and V. Chandra, Bulk viscosity of a hot QCD/ QGP medium in strong magnetic field within relaxationtime approximation, Phys. Rev. D 97, 116008 (2018).

[51] M. Kurian and V. Chandra, Effective description of hot QCD medium in strong magnetic field and longitudinal conductivity, Phys. Rev. D 96, 114026 (2017).

[52] B. Singh, L. Thakur, and H. Mishra, Heavy quark complex potential in a strongly magnetized hot QGP medium, Phys. Rev. D 97, 096011 (2018).

[53] M. Hasan, B. K. Patra, B. Chatterjee, and P. Bagchi, Landau Damping in a strong magnetic field: Dissociation of quarkonia, arXiv:1802.06874.

[54] K. Tuchin, Electromagnetic radiation by quark-gluon plasma in a magnetic field, Phys. Rev. C 87, 024912 (2013).

[55] A. Bandyopadhyay, C. A. Islam, and M. G. Mustafa, Electromagnetic spectral properties and Debye screening of a strongly magnetized hot medium, Phys. Rev. D 94, 114034 (2016).

[56] N. Sadooghi and F. Taghinavaz, Dilepton production rate in a hot and magnetized quark-gluon plasma, Ann. Phys. (Amsterdam) 376, 218 (2017).

[57] K. Tuchin, Magnetic contribution to dilepton production in heavy-ion collisions, Phys. Rev. C 88, 024910 (2013).

[58] K. Tuchin, Particle production in strong electromagnetic fields in relativistic heavy-ion collisions, Adv. High Energy Phys. 2013, 1 (2013).

[59] A. Bandyopadhyay and S. Mallik, Effect of magnetic field on dilepton production in a hot plasma, Phys. Rev. D 95, 074019 (2017).

[60] K. A. Mamo, Enhanced thermal photon and dilepton production in strongly coupled $\mathcal{N}=4 \mathrm{SYM}$ plasma in strong magnetic field, J. High Energy Phys. 08 (2013) 083.

[61] C. R. Allton, M. Doring, S. Ejiri, S. J. Hands, O. Kaczmarek, F. Karsch, E. Laermann, and K. Redlich, Thermodynamics of two flavor QCD to sixth order in quark chemical potential, Phys. Rev. D 71, 054508 (2005).
[62] E. V. Shuryak, Theory of hadronic plasma, Zh. Eksp. Teor. Fiz. 74, 408 (1978) [Sov. Phys. JETP 47, 212 (1978)].

[63] S. A. Chin, Transition to hot quark matter in relativistic heavy ion collision, Phys. Lett. 78B, 552 (1978).

[64] J. I. Kapusta, Quantum chromodynamics at high temperature, Nucl. Phys. B148, 461 (1979).

[65] T. Toimela, The next term in the thermodynamic potential of QCD, Phys. Lett. 124B, 407 (1983).

[66] P. B. Arnold and C. X. Zhai, The three loop free energy for pure gauge QCD, Phys. Rev. D 50, 7603 (1994).

[67] P. B. Arnold and C. x. Zhai, The three loop free energy for high temperature QED and QCD with fermions, Phys. Rev. D 51, 1906 (1995).

[68] C. x. Zhai and B. M. Kastening, The free energy of hot gauge theories with fermions through $\mathrm{g} * * 5$, Phys. Rev. D 52, 7232 (1995).

[69] J. O. Andersen, E. Braaten, and M. Strickland, Hard Thermal Loop Resummation of the Free Energy of a Hot Gluon Plasma, Phys. Rev. Lett. 83, 2139 (1999).

[70] J. O. Andersen, E. Braaten, and M. Strickland, Hard thermal loop resummation of the thermodynamics of a hot gluon plasma, Phys. Rev. D 61, 014017 (1999).

[71] J. O. Andersen, E. Braaten, and M. Strickland, Hard thermal loop resummation of the free energy of a hot quark: Gluon plasma, Phys. Rev. D 61, 074016 (2000).

[72] J. O. Andersen, S. Mogliacci, N. Su, and A. Vuorinen, Quark number susceptibilities from resummed perturbation theory, Phys. Rev. D 87, 074003 (2013).

[73] S. Mogliacci, J. O. Andersen, M. Strickland, N. Su, and A. Vuorinen, Equation of state of hot and dense QCD: Resummed perturbation theory confronts lattice data, J. High Energy Phys. 12 (2013) 055.

[74] N. Haque, M. G. Mustafa, and M. H. Thoma, Conserved density fluctuation and temporal correlation function in HTL perturbation theory, Phys. Rev. D 84, 054009 (2011).

[75] N. Haque and M. G. Mustafa, Quark number susceptibility and thermodynamics in HTL approximation, Nucl. Phys. A862-863, 271 (2011).

[76] N. Haque and M. G. Mustafa, A modified hard thermal loop perturbation theory, arXiv:1007.2076.

[77] N. Haque, Quark mass dependent collective excitations and quark number susceptibilities within the hard thermal loop approximation, Phys. Rev. D 98, 014013 (2018).

[78] J. O. Andersen, E. Braaten, E. Petitgirard, and M. Strickland, HTL perturbation theory to two loops, Phys. Rev. D 66, 085016 (2002).

[79] J. O. Andersen, E. Petitgirard, and M. Strickland, Two loop HTL thermodynamics with quarks, Phys. Rev. D 70, 045001 (2004).

[80] N. Haque, M. G. Mustafa, and M. Strickland, Two-loop HTL pressure at finite temperature and chemical potential, Phys. Rev. D 87, 105007 (2013).

[81] N. Haque, M. G. Mustafa, and M. Strickland, Quark number susceptibilities from two-loop hard thermal loop perturbation theory, J. High Energy Phys. 07 (2013) 184.

[82] J. O. Andersen, M. Strickland, and N. Su, Gluon Thermodynamics at Intermediate Coupling, Phys. Rev. Lett. 104, 122003 (2010). 
[83] J. O. Andersen, M. Strickland, and N. Su, Three-loop HTL gluon thermodynamics at intermediate coupling, J. High Energy Phys. 08 (2010) 113.

[84] J. O. Andersen, L. E. Leganger, M. Strickland, and N. Su, NNLO hard-thermal-loop thermodynamics for QCD, Phys. Lett. B 696, 468 (2011).

[85] J. O. Andersen, L. E. Leganger, M. Strickland, and N. Su, Three-loop HTL QCD thermodynamics, J. High Energy Phys. 08 (2011) 053.

[86] J. O. Andersen, L. E. Leganger, M. Strickland, and N. Su, The QCD trace anomaly, Phys. Rev. D 84, 087703 (2011).

[87] N. Haque, J. O. Andersen, M. G. Mustafa, M. Strickland, and N. Su, Three-loop HTLpt pressure and susceptibilities at finite temperature and density, Phys. Rev. D 89, 061701 (2014).

[88] N. Haque, A. Bandyopadhyay, J. O. Andersen, M. G. Mustafa, M. Strickland, and N. Su, Three-loop HTLpt thermodynamics at finite temperature and chemical potential, J. High Energy Phys. 05 (2014) 027.

[89] N. Haque, Finite temperature QCD four-point function in the presence of a weak magnetic field within the hard thermal loop approximation, Phys. Rev. D 96, 014019 (2017).

[90] A. Ayala, J. J. Cobos-Martínez, M. Loewe, M. E. TejedaYeomans, and R. Zamora, Finite temperature quark-gluon vertex with a magnetic field in the hard thermal loop approximation, Phys. Rev. D 91, 016007 (2015).

[91] V. Canuto and H. Y. Chiu, Quantum theory of an electron gas in intense magnetic fields, Phys. Rev. 173, 1210 (1968).

[92] M. Chaichian, S. S. Masood, C. Montonen, A. Perez Martinez, and H. Perez Rojas, Quantum Magnetic and Gravitational Collapse, Phys. Rev. Lett. 84, 5261 (2000).

[93] A. Broderick, M. Prakash, and J. M. Lattimer, The equation of state of neutron star matter in strong magnetic fields, Astrophys. J. 537, 351 (2000).

[94] A. P. Martinez, H. P. Rojas, and H. J. Mosquera Cuesta, Magnetic collapse of a neutron gas: Can magnetars indeed be formed, Eur. Phys. J. C 29, 111 (2003).
[95] A. Perez Martinez, H. Perez Rojas, and H. Mosquera Cuesta, Anisotropic pressures in very dense magnetized matter, Int. J. Mod. Phys. D 17, 2107 (2008).

[96] G. S. Bali, F. Bruckmann, G. Endrödi, S. D. Katz, and A. Schäfer, The QCD equation of state in background magnetic fields, J. High Energy Phys. 08 (2014) 177.

[97] B. Karmakar, R. Ghosh, A. Bandyopadhyay, N. Haque, and M. G. Mustafa, Anisotropic pressure of deconfined QCD matter in the presence of a strong magnetic field within oneloop approximation, Phys. Rev. D 99, 094002 (2019).

[98] T. Chyi, C-W. Hwang, W. F. Kao, L-L. Lin, K-W. Ng, and J-J. Tseng, Weak-field expansion for processes in a homogeneous background magnetic field, Phys. Rev. D 62, 105014 (2000).

[99] A. Ayala, A. Sanchez, G Piccinelli, and S. Sahu, Effective potential at finite temperature in a constant magnetic field: Ring diagrams in a scalar theory, Phys. Rev. D 71, 023004 (2005).

[100] L. Dolan and R. Jackiw, Symmetry behavior at finite temperature, Phys. Rev. D 9, 3320 (1974).

[101] J. I. Kapusta and C. Gale, Finite Temperature Field Theory, 2nd ed. (Cambridge University Press, Cambridge, England, 2006).

[102] M. Le Bellac, Thermal Field Theory, Cambridge Monographs on Mathematical Physics (Cambridge University Press, Cambridge, England, 1996).

[103] J. Alexandre, Vacuum polarization in thermal QED with an external magnetic field, Phys. Rev. D 63, 073010 (2001).

[104] M. Hasan, B. Chatterjee, and B. K. Patra, Heavy quark potential in a static and strong homogeneous magnetic field, Eur. Phys. J. C 77, 767 (2017).

[105] A. Ayala, C. A. Dominguez, S. Hernandez-Ortiz, L. A. Hernandez, M. Loewe, D. Manreza Paret, and R. Zamora, Thermomagnetic evolution of the QCD strong coupling, Phys. Rev. D 98, 031501 (2018).

[106] J. Beringer et al. (Particle Data Group), Review of particle physics, Phys. Rev. D 86, 010001 (2012).

[107] J. O. Andersen, E. Braaten, and M. Strickland, Hardthermal-loop resummation of the free energy of a quarkgluon plasma, Phys. Rev. D 61, 074016 (2000). 